\title{
On the asymptotic behaviour of anisotropic energies arising in the cardiac bidomain model
}

\author{
Luigi Ambrosio ${ }^{\dagger}$ \\ Scuola Normale Superiore, Pisa, Piazza dei Cavalieri 7, 56126 Pisa, Italy \\ AND \\ Piero Colli Franzone ${ }^{\ddagger}$ AND GIUSEPPE SAVARÉ $\$$ \\ Dipartimento di Matematica 'F. Casorati', Università di Pavia and Istituto di Analisi Numerica \\ del CNR, via Ferrata 1, 27100 Pavia, Italy \\ [Received 1 March 1999 and in revised form 16 February 2000]

\begin{abstract}
We study the $\Gamma$-convergence of a family of vectorial integral functionals, which are the sum of a vanishing anisotropic quadratic form in the gradients and a penalizing double-well potential depending only on a linear combination of the components of their argument. This particular feature arises from the study of the so-called 'bidomain model' for the cardiac electric field; one of its consequences is that the $L^{1}$-norm of a minimizing sequence can be unbounded and therefore a lack of coercivity occurs. We characterize the $\Gamma$-limit as a surface integral functional, whose integrand is a convex function of the normal and can be computed by solving a localized minimization problem.
\end{abstract}

\section{Introduction}

The aim of this paper is to investigate, in the framework of $\Gamma$-convergence theory, the asymptotic behaviour, as $\varepsilon \downarrow 0$, of the family of vectorial integral functionals

$$
F^{\varepsilon}(\boldsymbol{u}):=\varepsilon \int_{\Omega} \boldsymbol{q}(x, \nabla \boldsymbol{u}(x)) \mathrm{d} x+\frac{1}{\varepsilon} \int_{\Omega} W(\ell(\boldsymbol{u})) \mathrm{d} x
$$

which are the sum of a vanishing term, associated to the anisotropic quadratic form $\boldsymbol{q}$ in the gradients $\nabla \boldsymbol{u}:=\left(\nabla u_{1}, \nabla u_{2}, \ldots, \nabla u_{m}\right)^{T}$, and a penalizing double-well potential $W$ depending only on a linear combination $\ell(\boldsymbol{u})$ of the components of their argument.

The particular structure of these functionals arises from the study of the so-called 'bidomain model' for the cardiac electric field. Before entering into the details of the mathematical formulation of the problem (which we postpone to the second part of this introduction), let us briefly recall the main features of the physical model; the reader interested only in the mathematical aspects of the theory can skip this section.

\footnotetext{
†E-mail: luigi@ambrosio.sns.it

E-mail: colli@ian.pv.cnr.it

${ }^{\S}$ E-mail: savare@ian.pv.cnr.it
} 


\subsection{The bidomain model of the bioelectric cardiac activity}

This relevant mathematical model concerns the description of the cardiac bioelectric activity during a heartbeat; here we give only a brief description of this fairly complex phenomenon in order to motivate the crucial properties of the bidomain model.

The cardiac tissue is composed of an arrangement of elongated cells connected end-to-end and/or side-to-side by junctions of low resistivity; a string of the elongated cells constitutes a fibre. At a cellular level the tissue can be viewed as composed of two volumes, the intra- and the extracellular spaces, separated by the cellular membrane. The potential jump $u$ through the membrane is called the transmembrane potential.

Starting from the sino-atrial node, which acts as a pacemaker, a front-like variation of the transmembrane potential $u$ spreads first in the atria and subsequently through the myocardium, with a very fast transition from the resting value $s_{-}$to the plateau value $s_{+}$. This phase constitutes the excitation or depolarization phase; it is followed by an interval of almost constant potential (refractory period) and a subsequent less rapid return to the initial state (repolarization). The time profile of the transmembrane potential $u$ is called the action potential.

The fibre structure of the myocardium is the main factor in the anisotropic conductivity in the cardiac tissue.

The whole process is quite complicated and is essentially due to current flows of sodium, potassium, and calcium ions through the cellular membrane separating the intra- $(i)$ and extracellular (e) media, and to their diffusion in these two conducting media.

At a macroscopic level, in spite of the discrete cellular structure, the cardiac tissue can be represented by a continuous model, called the bidomain model (see e.g. [12, 21, 29] and also [25]), which attempts to describe the averaged electric potentials and current flows inside (intra) and outside (extracellular space) the cardiac cells. In this representation,

$$
\Omega \subset \mathbb{R}^{3} \text { is the physical region occupied by the heart }
$$

and it coincides with the $i$ and $e$ domains, which are two interpenetrating and superimposed continua connected at each point by the cardiac cellular membrane; $u^{i}, u^{e}$ are the corresponding intra- and extracellular electric potentials, respectively,whose difference $u=u^{i}-u^{e}$ is just the transmembrane potential.

The anisotropy of the $(i)-(e)$ media depends on the fibre structure of the myocardium. At the macroscopic level the fibres are regular curves, whose unit tangent vector at the point $x$ is denoted by $\boldsymbol{a}=\boldsymbol{a}(x)$. Denoting by $\sigma_{l}^{i, e}(x), \sigma_{t}^{i, e}(x)$ the conductivity coefficients along and across the fibre direction at a point $x$ and always assuming axial simmetry for $\sigma_{t}^{i, e}$, the conductivity tensors $A^{i, e}$ in the media $(i),(e)$, can be expressed by

$$
A^{i, e}(x)=\sigma_{t}^{i, e} I+\left(\sigma_{l}^{i, e}-\sigma_{t}^{i, e}\right) \boldsymbol{a a ^ { T }},
$$

and they are

$$
\begin{aligned}
& \text { symmetric, positive definite, continuous } \\
& \text { conductivity tensors } A^{i, e}: \bar{\Omega} \rightarrow \mathbb{M}^{3 \times 3} \text {. }
\end{aligned}
$$

To the potentials $u^{i}, u^{e}$ are associated the current densities

$$
\boldsymbol{J}^{i}=-M^{i} \nabla u^{i}, \quad \boldsymbol{J}^{e}=-M^{e} \nabla u^{e} .
$$


Since induction effects are negligible, the current field can be considered quasi-static; therefore we have

$$
\operatorname{div}\left(\boldsymbol{J}^{i}+\boldsymbol{J}^{e}\right)=0 \quad \text { in } \Omega .
$$

The currents $\boldsymbol{J}^{i}, \boldsymbol{J}^{e}$ are related to the membrane current per unit volume $i^{m}$ by the conservation law $i^{m}=\operatorname{div} \boldsymbol{J}^{e}=-\operatorname{div} \boldsymbol{J}^{i} ;$ for an insulated myocardial volume (i.e. condition $\boldsymbol{n}^{T} \boldsymbol{J}^{e}=\boldsymbol{n}^{T} \boldsymbol{J}^{i}=0$ is satisfied on the heart surface $\partial \Omega$ ), applying the nonlinear cable theory for the membrane current (see [22]) we have, in terms of potentials, the following reaction-diffusion (R-D) system:

$$
\left\{\begin{array}{lll}
i^{m}=c^{m} \partial_{t} u+i_{\text {ion }}, & \text { with } u:=u^{i}-u^{e}, & \\
\operatorname{div} M^{i} \nabla u^{i}=i^{m}, & \operatorname{div} M^{e} \nabla u^{e}=-i^{m} & \text { in } \Omega, \\
\boldsymbol{n}^{T} M^{i} \nabla u^{i}=0, & \boldsymbol{n}^{T} M^{e} \nabla u^{e}=0 & \text { on } \partial \Omega,
\end{array}\right.
$$

where $c^{m}$ is the membrane capacitance per unit volume and $i_{i o n}$ results from the combination of various membrane currents related to ionic fluxes. The initial condition $u(x, 0)=u^{0}(x)$ must be supplied to close the differential problem (1.2).

The coupling of the two equations is featured by a degenerate temporal structure: we refer to [14] for the mathematical treatment of this kind of degenerate R-D system.

The bidomain model (1.2) represents a macroscopic description of the entire electric behaviour of the cardiac tissue, i.e. depolarization and repolarization phases. In problem (1.2) the transmembrane potential $u$ exhibits a steep propagating layer, having a thickness of about $0.5 \mathrm{~mm}$, spreading throughout the myocardium with an upstroke phase lasting about $1 \mathrm{~ms}$ during the depolarization process.

The FitzHugh-Nagumo approximation For qualitative studies or macroscopic simulations we can disregard the fine details associated to the ionic fluxes through the cellular membrane, considering instead a simplified model of the membrane current called the FitzHugh-Nagumo approximation $[12,18]$. In this model the ionic current is represented by

$$
\left\{\begin{array}{l}
i_{i o n}=i_{\text {ion }}(u, w):=F(u)+\delta w, \\
\partial_{t} w=\beta u-\gamma w
\end{array} \quad \text { in } \Omega,\right.
$$

where $\delta, \gamma, \beta$ are positive constants, $w$ is a recovery variable, and $F$ is a continuous cubic-like function. The function $F(u)$ describes an instantaneous current-voltage membrane relationship, which mimics the ionic membrane activation mechanism; $F(u)$ results in a cubic-like function having $s_{-}<s_{c}<s_{+}$as zeros, with $s_{ \pm}$stable zeros, and $\int_{s_{-}}^{s_{+}} F(u) \mathrm{d} v<0$.

Although this simplified ionic model is not suitable for a quantitative detailed study at a fine scale of the upstroke of the action potential $u$ through the excitation wavefront, it is nevertheless appropriate for gaining general insight into wave propagation in the cardiac excitable medium, especially if we are interested in the large-scale behaviour of the front-like solution. Then during the excitation phase in a fully recovered tissue, we can neglect the recovery variable $w$, hence in its simplest form the model governing the excitation bioelectric activity of the cardiac tissue $\Omega$ is given by the R-D problem (1.2) with $i_{\text {ion }}=F(v)$.

Proceeding with a suitable re-scaling, as in $[12,13]$, the non-dimensional form of the 
macroscopic bidomain model can be written as the following singularly perturbed R-D system:

$$
\left\{\begin{array}{l}
\partial_{t}\left(u_{\varepsilon}^{i}-u_{\varepsilon}^{e}\right)-\varepsilon \operatorname{div} M^{i} \nabla u_{\varepsilon}^{i}+\frac{1}{\varepsilon} F\left(u_{\varepsilon}^{i}-u_{\varepsilon}^{e}\right)=0 \\
\partial_{t}\left(u_{\varepsilon}^{e}-u_{\varepsilon}^{i}\right)-\varepsilon \operatorname{div} M^{e} \nabla u_{\varepsilon}^{e}-\frac{1}{\varepsilon} F\left(u_{\varepsilon}^{i}-u_{\varepsilon}^{e}\right)=0
\end{array}\right.
$$

where the order of magnitude of the dimensionless parameter $\varepsilon$ is $10^{-3}-10^{-2}$.

Variational structure and Lyapunov functional If we introduce the couple

$$
\boldsymbol{u}:=\left(u_{\varepsilon}^{i},-u_{\varepsilon}^{e}\right)
$$

of the intra- and (the opposite of) the extracellular potentials, and a potential $W: \mathbb{R} \rightarrow \mathbb{R}$ such that $W^{\prime}=F$, then the degenerate reaction-diffusion system associated to (1.2) in the couple of unknowns $\boldsymbol{u}:=\left(u^{i},-u^{e}\right)$ can be obtained by taking the gradient flow of the Lyapunov functional

$$
\begin{aligned}
F^{\varepsilon}(\boldsymbol{u}) & :=\varepsilon \int_{\Omega}\left(\left\langle A^{i} \nabla u^{i}, \nabla u^{i}\right\rangle+\left\langle A^{e} \nabla u^{e}, \nabla u^{e}\right\rangle\right) \mathrm{d} x \\
& +\frac{1}{\varepsilon} \int_{\Omega} W\left(u_{\varepsilon}^{i}-u_{\varepsilon}^{e}\right) \mathrm{d} x,
\end{aligned}
$$

with respect to the positive but degenerate bilinear form in $L^{2}\left(\Omega ; \mathbb{R}^{2}\right)$

$$
b(\boldsymbol{v}, \boldsymbol{w}):=\int_{\Omega}\left(v^{i}-v^{e}\right)\left(w^{i}-w^{e}\right) \mathrm{d} x, \quad \boldsymbol{v}=\left(v^{i},-v^{e}\right), \quad \boldsymbol{w}=\left(w^{i},-w^{e}\right) .
$$

This yields the following system of variational evolution equations:

$$
b\left(\partial_{t} \boldsymbol{u}, \boldsymbol{v}\right)+\delta F^{\varepsilon}(\boldsymbol{u}, \boldsymbol{v})=0 \quad \forall \boldsymbol{v} \in H^{1}\left(\Omega ; \mathbb{R}^{2}\right),
$$

where $\delta F^{\varepsilon}(\boldsymbol{u}, \cdot)$ is the Euler-Lagrange first variation of the functional $F^{\varepsilon}$ [19: pp. 9-10], which is the variational formulation of (1.4).

If we call $\ell$ the linear form

$$
\ell(\boldsymbol{u}):=u_{\varepsilon}^{i}-u_{\varepsilon}^{e}=u
$$

which defines the transmembrane potential, and $q^{i, e}, \boldsymbol{q}$ the quadratic forms associated to $A^{i, e}$,

$$
\begin{gathered}
q^{i, e}(x, \xi):=\left\langle A^{i, e}(x) \xi, \xi\right\rangle, \quad \forall \xi \in \mathbb{R}^{3}, \\
\boldsymbol{q}(x, \mathfrak{a}):=q^{i}\left(x, \mathfrak{a}^{i}\right)+q^{e}\left(x, \mathfrak{a}^{e}\right), \quad \mathfrak{a}=\left(\mathfrak{a}^{i}, \mathfrak{a}^{e}\right)^{T} \in \mathbb{M}^{2 \times 3},
\end{gathered}
$$

then the Lyapunov functional $\left(B_{4}\right)$ has exactly the same structure as (1.1).

Formal asymptotics and anisotropic evolution of the interface In the bidomain model (1.4) the reactive term $F$ is bistable and depends, instantaneously, only on the transmembrane potential. Thus the nonlinear potential energy $W$ depends only on $u=u^{i}-u^{e}$, which is a fundamental feature of the 
model and reflects the degeneracy of the system; moreover $W$ exhibits two minima with different depths.

This singular perturbation structure implies that $u_{\varepsilon}^{i}, u_{\varepsilon}^{e}$ diffuse quite slowly while the reaction takes place much faster, and from the layer analysis the development of an interface associated with a travelling wavefront solution is expected. Exploiting the singular perturbation approach we derive anisotropic geometric evolution laws capturing the asymptotic behaviour of travelling wavefront solutions of the R-D system (1.2) (see [5, 12, 13, 23-25]); for isotropic media and full FitzhughNagumo dynamics (1.3) see [30].

The excitation wavefront $S_{\varepsilon}(t)$ is represented by the level surface of the transmembrane potential $v_{\varepsilon}=u_{\varepsilon}^{i}-u_{\varepsilon}^{e}$ of value $\left(s_{-}+s_{+}\right) / 2$, i.e.

$$
S_{\varepsilon}(t)=\left\{x \in \Omega, \quad v_{\varepsilon}(x, t)=\left(s_{-}+s_{+}\right) / 2\right\} .
$$

We already introduced $\left(B_{6}\right)$ the quadratic forms $q^{i, e}(x, \xi)$ which, if $\xi$ is a unit vector, represent the conductivity coefficient at a point $x$ in the intra- and extracellular medium measured along the direction $\xi$. We call $\bar{q}$ the harmonic mean of the quadratic forms associated to the conductivity tensors $M^{i, e}$, i.e.

$$
\bar{q}(x, \xi):=\left(q^{i}(x, \xi)^{-1}+q^{e}(x, \xi)^{-1}\right)^{-1},
$$

and we introduce the surface energy density

$$
\bar{\phi}(x, \xi):=\sqrt{\bar{q}(x, \xi)} .
$$

Let $(\mathrm{c}, a)$ be the unique bounded solution of the eigenvalue problem

$$
\left\{\begin{array}{l}
-a^{\prime \prime}+c a^{\prime}+f(a)=0 \\
a( \pm \infty)=s_{+} \text {or } s_{-}, \quad a(0)=\left(s_{-}+s_{+}\right) / 2
\end{array}\right.
$$

and let $v(x)$ be the Euclidean unit vector normal to the wavefront $S_{\varepsilon}(t)$ oriented toward the resting region. In [5] the vector $n_{\bar{\phi}}=D_{\xi} \bar{\phi}(x, v(x))$ and the moving reference $(s(t), y)$ defined by stretching the space coordinate along the $n_{\bar{\phi}}$ direction were introduced, i.e.

$$
y=\frac{\eta}{\varepsilon}, \quad x=s(t)+\eta n_{\bar{\phi}}(s(t)), \quad \text { for } s(t) \in S_{\varepsilon}(t) .
$$

Using this moving frame, in [5: Appendix B] formal asymptotic expansions of $u_{\varepsilon}^{i}, u_{\varepsilon}^{e}$, and of $v_{\varepsilon}$, in power of $\varepsilon$, were developed showing that the front associated to (1.6) moves along the relative normal vector $n_{\bar{\phi}}$ with velocity $V_{\epsilon}\left(n_{\bar{\phi}}\right)$ given at any $s(t) \in S_{\varepsilon}(t)$ by

$$
V_{\epsilon}\left(n_{\bar{\phi}}\right)=c-\varepsilon \operatorname{div} n_{\bar{\phi}}+\mathcal{O}\left(\varepsilon^{2}\right),
$$

where $c$ is related to the travelling wave solution $a$ of (1.9). Moreover the velocity $V_{\epsilon}(v)$ measured along the Euclidean normal direction $v$ of $S_{\varepsilon}(t)$ is given by

$$
V_{\epsilon}(v)=\bar{\phi}(s, v)\left(c-\varepsilon \operatorname{div} D_{\xi} \bar{\phi}(s, v)\right)+\mathcal{O}\left(\varepsilon^{2}\right) .
$$

Therefore dropping $\mathcal{O}\left(\varepsilon^{2}\right)$ terms, the front behaves as an hypersurface $S(t)$, propagating with the anisotropic geometric law with normal velocity $V(\nu)$ given by

$$
V(v)=\bar{\phi}(s, v)\left(c-\varepsilon \operatorname{div} D_{\xi} \bar{\phi}(s, v)\right) .
$$

Equations of this type are also called eikonal-curvature models since, with respect to a suitable Finsler metric, $K_{\bar{\phi}}:=\operatorname{div}_{S(t)} n_{\bar{\phi}}$ is the anisotropic mean curvature of $S_{\varepsilon}(t)$ (see [5-7]). 
Anisotropic surface energy in the stationary case Since $K_{\bar{\phi}}$ corresponds to the first variation of the anisotropic surface energy associated to $\bar{\phi}$, in order to characterize the form of the anisotropic curvature term we study the asymptotic limit, as $\varepsilon \downarrow 0$, of the stationary problem associated to a singular R-D system (1.2) for a function $F=W^{\prime}$, where now $W$ is a potential having wells of equal depth; more precisely, we try to characterize the this limit behaviour by carefully studying the $\Gamma$-convergence of the underlying potentials $\left(B_{4}\right)$.

Therefore, our contribution is intended as a first step towards a rigorous mathematical theory describing and justifying the right asymptotic motion law of the cardiac excitation wavefronts. We believe that understanding the limit of Lyapunov functionals associated to the family $\left(B_{4}\right)$ gives insights about the correct choice of the anisotropic surface energy driving the propagation of the fronts.

\subsection{Mathematical formulation of the problem and main results}

In a Lipschitz and bounded open set $\Omega$ of $\mathbb{R}^{n}$, let us consider a continuous family of quadratic forms

$$
\boldsymbol{q}: \bar{\Omega} \times \mathbb{M}^{m \times n} \rightarrow \mathbb{R},
$$

$\mathbb{M}^{m \times n}$ being the vector space of the $m \times n$ matrices satisfying the usual uniform ellipticity condition

$$
\exists \lambda, \Lambda>0: \quad \lambda|\mathfrak{a}|^{2} \leqslant \boldsymbol{q}(x, \mathfrak{a}) \leqslant \Lambda|\mathfrak{a}|^{2} \quad \forall x \in \bar{\Omega}, \forall \mathfrak{a} \in \mathbb{M}^{m \times n} .
$$

Let $W: \mathbb{R} \rightarrow[0,+\infty]$ be a continuous double-well potential, such that

$$
\begin{gathered}
W(s)=0 \Leftrightarrow s \in T:=\left\{s_{-}, s_{+}\right\} \subset \mathbb{R}, \\
0<\liminf _{|s| \uparrow+\infty} \frac{W(s)}{|s|^{p_{0}}} \leqslant \limsup _{|s| \uparrow+\infty} \frac{W(s)}{|s|^{p_{0}}}<+\infty \quad \text { for some } p_{0} \geqslant 2,
\end{gathered}
$$

and let us consider the family of integrands

$$
\begin{gathered}
f^{\varepsilon}(x, \boldsymbol{s}, \mathfrak{a}):=\varepsilon \boldsymbol{q}(x, \mathfrak{a})+\frac{1}{\varepsilon} \tilde{W}(\boldsymbol{s}), \quad(x, \boldsymbol{s}, \mathfrak{a}) \in \Omega \times \mathbb{R}^{m} \times \mathbb{M}^{m \times n}, \\
\tilde{W}(\boldsymbol{s}):=W(\ell(\boldsymbol{s})), \quad \ell(\boldsymbol{s}):=\langle\ell, \boldsymbol{s}\rangle, \quad \ell:=\left(\ell_{1}, \ldots, \ell_{m}\right) \neq 0 .
\end{gathered}
$$

As we quoted at the beginning of this introduction, we want to study the $\Gamma$-convergence, as $\varepsilon \downarrow 0$, of the family of vectorial integral functionals in $L^{2}\left(\Omega ; \mathbb{R}^{m}\right)$

$$
\begin{aligned}
F^{\varepsilon}(\boldsymbol{u}) & :=\varepsilon \int_{\Omega} \boldsymbol{q}(x, \nabla \boldsymbol{u}(x)) \mathrm{d} x+\frac{1}{\varepsilon} \int_{\Omega} W(\ell(\boldsymbol{u})) \mathrm{d} x \\
& =\int_{\Omega} f^{\varepsilon}(x, \boldsymbol{u}, \nabla \boldsymbol{u}) \mathrm{d} x, \quad \nabla \boldsymbol{u}:=\left(\nabla u_{1}, \nabla u_{2}, \ldots, \nabla u_{m}\right)^{T},
\end{aligned}
$$

where, as usual, we set $F^{\varepsilon}(\boldsymbol{u}):=+\infty$ if $\boldsymbol{u} \notin H^{1}\left(\Omega ; \mathbb{R}^{m}\right) \cap L^{p_{0}}\left(\Omega ; \mathbb{R}^{m}\right)$. We point out that problem (1.17) is vectorial and anisotropic, with the penalizing potential only dependent on a linear combination of the components of $\boldsymbol{u}$.

After a brief overview of the available results in the literature for singular perturbation problems of anisotropic, vectorial and coercive type, in the remaining part of this introduction we will present the statements of the main theorems of the paper, highlighting the difficulties and the differences in dealing with our class of problems, which do not fit into the standard theory due to the lack of coercivity. 
Anisotropic singular perturbations: the coercive case The $\Gamma$-convergence of the integral functionals (1.17), where $f^{\varepsilon}$ has a structure like (1.16a), has been deeply studied under various assumptions on $f^{\varepsilon}$, starting from the papers $[26,27]$ in the isotropic scalar case. These results were generalized to anisotropic (and not necessarily of quadratic type) functions in [8,28] (cf. also [7] for a different approach in the context of Finsler metrics), and to a very general anisotropic vectorial framework in [4].

In all these cases it is assumed that the potential $\tilde{W}$, which could also depend on the $x$-variable, has only two distinct minimum points w.r.t. $s$ say

$$
\boldsymbol{s}_{-}, \boldsymbol{s}_{+} \in \mathbb{R}^{m} .
$$

(cf. [1,3] for the generalization to the case of a finite or compact minimum set).

It is then possible to show that:

- there exists the $\Gamma$-limit $F$ of $F^{\varepsilon}$ as $\varepsilon$ goes to 0 , with respect to the strong topology of $L^{p}\left(\Omega ; \mathbb{R}^{m}\right)$, $1 \leqslant p \leqslant p_{0}$

- $\quad F(\boldsymbol{u})$ is finite if and only if $\boldsymbol{u}$ is a function of bounded variation in $\Omega$ assuming only the values $\boldsymbol{s}_{-}, \boldsymbol{s}_{+}$a.e.;

- on these functions $F$ can be represented as an integral functional on the discontinuity surface of $\boldsymbol{u}$ as

$$
F(\boldsymbol{u})=\int_{S_{\boldsymbol{u}}^{*}} \varphi\left(x, v_{\boldsymbol{u}}(x)\right) \mathrm{d} H^{n-1}(x),
$$

where ${ }^{\dagger} S_{u}^{*}$ is the common reduced boundary, in the sense of De Giorgi, of the two level sets of $\boldsymbol{u}, \boldsymbol{v}_{\boldsymbol{u}}$ is the approximate unit normal, and $\{\varphi(x, \cdot)\}_{x \in \Omega}$ is a suitable family of norms on $\mathbb{R}^{n}$.

The precise calculation of these surface energy densities is not easy in the anisotropic vectorial case: they can be characterized by considering the 'minimal cost' of a transition from $\boldsymbol{s}_{-}$to $\boldsymbol{s}_{+}$in a cylinder across a unitary planar surface perpendicular to a direction $v$.

More precisely, let us first describe what we mean by the term cylinder: we fix

$$
\text { an hyperplane } \pi \subset \mathbb{R}^{n} \text {, a section } \Sigma \subset \pi,
$$

a vector $\eta \notin \pi$, and an open interval (possibly unbounded) $(a, b) \subset \mathbb{R}$.

A general cylinder with section $\Sigma$ and direction $\eta$ is $\Sigma(a, b ; \eta) \subset \mathbb{R}^{n}$ defined by

$$
\Sigma(a, b ; \eta)=\{x+t \eta: x \in \Sigma, t \in(a, b)\} .
$$

We say that a set $Q:=\Sigma(a, b ; \eta) \subset \mathbb{R}^{n}$ is an admissible cylinder for $v \in \mathbb{S}^{n-1}$ if

$$
\begin{gathered}
\pi \text { is orthogonal to } \nu, \quad a<0<b, \\
\Sigma=\left\{\sum_{i=1}^{n-1} r_{i} \eta_{i}:\left|r_{i}\right|<1 / 2\right\} \text { for a basis }\left\{\eta_{1}, \eta_{2}, \ldots \eta_{n-1}\right\} \text { of } \pi \\
\Sigma \text { has unitary }(n-1) \text {-Hausdorff measure. }
\end{gathered}
$$

\footnotetext{
The precise definitions of these and other concepts are given in the next section.
} 
We denote the upper and lower bases of $Q$ by

$$
\Sigma_{+}:=\{x+b \eta: x \in \Sigma\}, \quad \Sigma_{-}:=\{x+a \eta: x \in \Sigma\}
$$

and we introduce the closed subspace of $H^{1}\left(Q ; \mathbb{R}^{2}\right)$

$$
\begin{gathered}
\mathcal{A}\left(Q ; \mathbb{R}^{m}\right):=\left\{\boldsymbol{u} \in H^{1}\left(Q ; \mathbb{R}^{m}\right): \boldsymbol{u}_{\left.\right|_{\partial Q} \text { depends only on }\langle x, v\rangle,}\right. \\
\left.\boldsymbol{u}(x)=\boldsymbol{s}_{ \pm} \text {on } \Sigma_{ \pm}\right\}
\end{gathered}
$$

Then $\phi$ admits the following expression (cf. [4])

$$
\varphi\left(x_{0}, v\right)=\inf \left\{\int_{Q} f^{\varepsilon}\left(x_{0}, \boldsymbol{u}, \nabla \boldsymbol{u}\right): \boldsymbol{u} \in \mathcal{A}\left(Q ; \mathbb{R}^{m}\right), \varepsilon>0\right\} .
$$

In the scalar case $m=1$, this expression can be further simplified, since it is possible to put the minimization w.r.t. $\varepsilon$ inside the integral and therefore to replace $f^{\varepsilon}$ with

$$
f(x, \boldsymbol{s}, \mathfrak{a}):=\inf _{\varepsilon>0} f^{\varepsilon}(x, \boldsymbol{s}, \mathfrak{a}) .
$$

If $f^{\varepsilon}$ has the structure (1.16a), where now $\mathfrak{a}$ is a vector of $\mathbb{R}^{n}, \boldsymbol{q}(x, \cdot)$ is a quadratic form in $\mathbb{R}^{n}$ and $s$ is a scalar, then

$$
f(x, s, \mathfrak{a})=2 \sqrt{\tilde{W}(\boldsymbol{s}) \boldsymbol{q}(x, \mathfrak{a})}
$$

and the calculus of $\phi$ can be reduced to a one-dimensional problem which can be solved explicitly, giving the formula

$$
\phi\left(x_{0}, v\right)=2 \sqrt{\boldsymbol{q}\left(x_{0}, v\right)} \int_{s_{-}}^{s_{+}} \sqrt{\tilde{W}(s)} \mathrm{d} s .
$$

The non-coercive case The fundamental difference between the families of functionals considered in all these contributions and the one considered in our paper lies in the structure of the set of minimum points of the potential

$$
\boldsymbol{w} \in \mathbb{R}^{m} \mapsto \tilde{W}(\boldsymbol{w})=W(\ell(\boldsymbol{w}))
$$

i.e.

$$
\text { the set } \arg \min \tilde{W}:=\left\{\boldsymbol{w} \in \mathbb{R}^{m}: \ell(\boldsymbol{w})=s_{ \pm}\right\},
$$

which is the union of two parallel hyperplanes. This fact reflects that a general minimizing sequence $\boldsymbol{u}^{\varepsilon}$ for $F^{\varepsilon}$ can be unbounded in $L^{2}\left(\Omega ; \mathbb{R}^{m}\right)$, and a priori only some compactness properties for the sum $\ell\left(\boldsymbol{u}^{\varepsilon}\right)$ could be expected: therefore, we are dealing with an anisotropic singular perturbation problem with a lack of coercivity. One of the main consequences is that the study of the $\Gamma$-convergence is strongly influenced by the choice of the topology, among the $L^{p}$ ones for $1 \leqslant p \leqslant p_{0}$, which in a certain sense has to supply the lack of the control on all the components of $\boldsymbol{u}$. Our first result concerns the $\Gamma$-convergence w.r.t. the strong topology of $L^{2}\left(\Omega ; \mathbb{R}^{m}\right)$. 
THEOREM 1.1 There exists the $\Gamma\left(L^{2}\left(\Omega ; \mathbb{R}^{m}\right)\right.$ )-limit $F$ of $F^{\varepsilon}$ (defined in (1.17)) as $\varepsilon$ goes to 0 ; $F(\boldsymbol{u})$ depends only on $u:=\ell(\boldsymbol{u})$, it is finite if and only if $u$ is a function of bounded variation in $\Omega$ assuming only the values $s_{-}, s_{+}$a.e., and on this kind of function $F$ can be represented as in (1.19), i.e.

$$
F(\boldsymbol{u})=\int_{S_{u}^{*}} \phi\left(x, v_{u}(x)\right) \mathrm{d} H^{n-1}(x) .
$$

In this case $\{\phi(x, \cdot)\}_{x \in \Omega}$ is the continuous family of norms on $\mathbb{R}^{n}$ characterized by

$$
\phi\left(x_{0}, \nu\right)=\inf \left\{\int_{Q} f^{\varepsilon}\left(x_{0}, \boldsymbol{u}, \nabla \boldsymbol{u}\right): \boldsymbol{u} \in \mathcal{B}\left(Q ; \mathbb{R}^{m}\right), \varepsilon>0\right\},
$$

where $f^{\varepsilon}$ is defined as in (1.16), $Q$ is an admissible set for $v$ as in (1.20), and

$$
\begin{gathered}
\mathcal{B}\left(Q ; \mathbb{R}^{m}\right):=\left\{\boldsymbol{u} \in H^{1}\left(Q ; \mathbb{R}^{m}\right): \boldsymbol{u}_{\left.\right|_{\partial Q} \text { depends only on }\langle x, v\rangle,}\right. \\
\left.\ell(\boldsymbol{u})(x)=s_{ \pm} \text {on } \Sigma_{ \pm}\right\} .
\end{gathered}
$$

It is well known that a $\Gamma$-convergence result as given above is equivalent to two different properties: on one hand, for every element $\boldsymbol{u} \in L^{2}\left(\Omega ; \mathbb{R}^{m}\right)$ we have to be able to exhibit a family $\boldsymbol{u}^{\varepsilon} \in$ $L^{2}\left(\Omega ; \mathbb{R}^{m}\right)$ such that, as $\varepsilon \downarrow 0$,

$$
\boldsymbol{u}^{\varepsilon} \rightarrow \boldsymbol{u} \quad \text { and } \quad F^{\varepsilon}\left(\boldsymbol{u}^{\varepsilon}\right) \rightarrow F(\boldsymbol{u})
$$

on the other hand, a kind of 'lower semicontinuity' property should also be proved, i.e.

$$
\boldsymbol{u}^{\varepsilon} \rightarrow \boldsymbol{u} \quad \text { in } L^{2}\left(\Omega ; \mathbb{R}^{m}\right) \quad \Rightarrow \quad \liminf _{\varepsilon \downarrow 0} F^{\varepsilon}\left(\boldsymbol{u}^{\varepsilon}\right) \geqslant F(\boldsymbol{u}) .
$$

The stronger the topology in (1.27a), the better it is; on the contrary, from the point of view of the convergence of minimizers, in (1.27b) it is important to replace the strong topology of $L^{2}\left(\Omega ; \mathbb{R}^{m}\right)$ with the weak one, whenever we know that a family $\boldsymbol{u}^{\varepsilon}$ (arising, e.g., from a suitable minimization problem) is only bounded in $L^{2}\left(\Omega ; \mathbb{R}^{m}\right)$ and not totally bounded.

Of course, a result of this type is related to the $\Gamma$-convergence w.r.t. the weak topology of $L^{2}\left(\Omega ; \mathbb{R}^{m}\right)$; since this topology does not satisfy the first axiom of countability, in order to avoid technical difficulties we limit ourselves to consider the $\Gamma$-convergence of $F^{\varepsilon}$ on bounded sets of $L^{2}\left(\Omega ; \mathbb{R}^{m}\right)$, which is sufficient for proving $(1.27 \mathrm{~b})$ in the weak convergence case.

THEOREM 1.2 For every family $\boldsymbol{u}^{\varepsilon} \in L^{2}\left(\Omega ; \mathbb{R}^{m}\right), \varepsilon>0$, we have

$$
\boldsymbol{u}^{\varepsilon} \rightarrow \boldsymbol{u} \quad \text { in } L^{2}\left(\Omega ; \mathbb{R}^{m}\right) \text { as } \varepsilon \downarrow 0 \Rightarrow \liminf _{\varepsilon \downarrow 0} F^{\varepsilon}\left(\boldsymbol{u}^{\varepsilon}\right) \geqslant F(\boldsymbol{u}) .
$$

Lower and upper bounds for $\phi$ It is natural to ask if it is possible to provide a more direct formula for $\phi$. We will show now two explicit lower and upper bounds for $\phi$, and we will analyse them more deeply in the case of the bidomain model.

A first simple strategy to get an explicit lower bound is to minimize the integrand in (1.17) among all the possible decompositions of $\nabla u$ into a linear combination of arbitrary $\mathbb{R}^{n}$-valued functions, instead of gradient vector fields. 
Since

$$
\ell(\boldsymbol{u})=\langle\ell, \boldsymbol{u}\rangle=u \Rightarrow \nabla \boldsymbol{u}^{T} \cdot \ell=\nabla u,
$$

we introduce the coercive quadratic form on $\mathbb{R}^{n}$

$$
\underline{q}(x, \xi):=\min \left\{\boldsymbol{q}(x, \mathfrak{a}): \mathfrak{a}^{T} \cdot \ell=\xi\right\},
$$

which obviously satisfies

$$
\underline{q}(x, \xi) \geqslant \frac{\lambda}{|\ell|^{2}}|\xi|^{2} \quad \forall \xi \in \mathbb{R}^{m}
$$

By (1.29) it is easy to see that

$$
\ell(\boldsymbol{u})=u \quad \Rightarrow \quad F^{\varepsilon}(\boldsymbol{u}) \geqslant \int_{\Omega}\left(\varepsilon \underline{q}(x, \nabla u(x))+\frac{1}{\varepsilon} W(u(x))\right) \mathrm{d} x .
$$

By applying the already quoted results on the $\Gamma$-convergence of singularly perturbed problems in the scalar case, we obtain the following.

Proposition 1.3 For every $x \in \Omega$ and $\xi \in \mathbb{R}^{n}$,

$$
\phi(x, \xi) \geqslant \underline{\phi}(x, \xi):=\gamma_{0} \sqrt{\underline{q}(x, \xi)}
$$

where $\gamma_{0}$ is defined by

$$
\gamma_{0}:=2 \int_{s_{-}}^{s_{+}} \sqrt{W(s)} \mathrm{d} s .
$$

On the other hand, an explicit upper bound for $\phi$ can be obtained if we limit the admissible functions to be globally dependent only on $\langle x, v\rangle$ in the evaluation of the infimum (1.25). This constraint reduces (1.25) to a one-dimensional problem.

When a function $\boldsymbol{u}: Q \rightarrow \mathbb{R}^{m}$ depends only on $\langle x, v\rangle$ and it satisfies $\ell(\boldsymbol{u})=u$, it is not difficult to see that ${ }^{\dagger}$

$$
\nabla \boldsymbol{u}=\boldsymbol{\eta} \otimes \nabla u \quad \text { for some } \boldsymbol{\eta} \text { with } \ell(\boldsymbol{\eta})=1 .
$$

Thus we introduce the function

$$
\bar{q}(x, \xi):=\min \left\{\boldsymbol{q}(x, \boldsymbol{\eta} \otimes \xi): \boldsymbol{\eta} \in \mathbb{R}^{m}, \ell(\boldsymbol{\eta})=1\right\},
$$

which satisfies the estimate from above

$$
\bar{q}(x, \xi) \leqslant \frac{\Lambda}{|\ell|^{2}}|\xi|^{2} \quad \forall \xi \in \mathbb{R}^{m},
$$

and we obtain the proposition 1.4

Proposition 1.4 For every $x \in \Omega$ and $\xi \in \mathbb{R}^{n}$,

$$
\phi(x, \xi) \leqslant \bar{\phi}(x, \xi):=\gamma_{0} \sqrt{\bar{q}(x, \xi)},
$$

where $\gamma_{0}$ is defined by (1.34).

\footnotetext{
We use the notation $\eta \otimes \xi:=\eta \cdot \xi^{T}$, so that, for every $v \in \mathbb{R}^{n}$
}

$$
(\boldsymbol{\eta} \otimes \xi) \nu=\boldsymbol{\eta}\langle\xi, \nu\rangle
$$


Non-local functionals and the behaviour of the potentials in the 'bidomain model'. Now we come back to the settings $\left(B_{1}-B_{6}\right)$, and we try to give a more detailed description of the behaviour of the potentials

$$
\boldsymbol{u}:=\left(u_{1}, u_{2}\right)=\left(u^{i},-u^{e}\right)
$$

in terms of the transmembrane potential $u:=u^{i}-u^{e}$. From the physical point of view, the dependence of $F$ only on $u$ given by (1.24) corresponds to the fact that only the evolution of the transmembrane potential $u$ behaves like a propagating front, whereas the properties of the potentials $u^{i}, u^{e}$ are much more complicated, since they are related to $u$ by a non-local dependence.

This non-local character can also be better understood, if we associate to $F^{\varepsilon}$ the functionals $G^{\varepsilon}$ depending only on $u$, which are obtained by minimizing $F^{\varepsilon}$ among all the possible decompositions of $u$ as the sum of two functions in $H^{1}(\Omega)$. First of all we define the quadratic functional $Q$ on $H^{1}(\Omega)$

$$
Q(u):=\min \left\{\int_{\Omega} \boldsymbol{q}(x, \nabla \boldsymbol{u}(x)) \mathrm{d} x: \boldsymbol{u} \in H^{1}\left(\Omega ; \mathbb{R}^{2}\right), u^{i}-u^{e}=u \text { in } \Omega\right\}
$$

and we set

$$
G^{\varepsilon}(u):=\varepsilon Q(u)+\frac{1}{\varepsilon} \int_{\Omega} W(u(x)) \mathrm{d} x \quad \forall u \in H^{1}(\Omega),
$$

with the usual convention to extend $G^{\varepsilon}$ to $L^{2}(\Omega)$. If we denote by $\mathcal{U}(u)$ the set of all $\boldsymbol{u} \in H^{1}\left(\Omega ; \mathbb{R}^{2}\right)$ which achieve the minimum in (1.38), we obviously have

$$
u^{i}-u^{e}=u \Rightarrow F^{\varepsilon}(\boldsymbol{u}) \geqslant G^{\varepsilon}(u), \quad \text { the equality holds iff } \boldsymbol{u} \in \mathcal{U}(u) .
$$

In a certain sense, substituting a generic couple $\boldsymbol{u}$ with $\overline{\boldsymbol{u}} \in \mathcal{U}\left(u^{i}-u^{e}\right)$ provides a natural renormalization of $F^{\varepsilon}(\boldsymbol{u})$. In this way, of course, we obtain coercive functionals, and it is easy to see that their $\Gamma$-convergence is not influenced by the choice of an $L^{p}(\Omega)$-topology (both strong and weak) if $1 \leqslant p \leqslant p_{0},{ }^{\dagger}$ since they satisfy

$$
\sup _{\varepsilon>0} G^{\varepsilon}\left(u^{\varepsilon}\right)<+\infty \Rightarrow\left\{u^{\varepsilon}\right\}_{\varepsilon>0} \text { is precompact in } L^{p_{0}}(\Omega) .
$$

However new difficulties arise since $G^{\varepsilon}$ are non-local. We shall see that good a priori $L^{2}$-estimates for $\overline{\boldsymbol{u}}$ in terms of $\boldsymbol{u}$ entail a strict relationship between the $\Gamma$-limits of both families and allow a slightly better refinement of Theorems 1.1 and 1.2.

Let us observe that $\boldsymbol{u} \in \mathcal{U}(u)$ iff $u^{i}-u^{e}=u$ and $u^{i}, u^{e}$ solve (the natural variational formulation of) the following elliptic equations

$$
\begin{aligned}
& \mathcal{L} u^{i}=\mathcal{L}^{e} u, \quad \text { in } \Omega, \quad \partial_{\overline{\boldsymbol{n}}} u^{i}=\partial_{\overline{\boldsymbol{n}}^{e}} u \quad \text { on } \partial \Omega, \\
& \mathcal{L} u^{e}=-\mathcal{L}^{i} u, \quad \text { in } \Omega, \quad \partial_{\bar{n}} u^{e}=-\partial_{\overline{\boldsymbol{n}}^{i}} u \quad \text { on } \partial \Omega,
\end{aligned}
$$

where $\mathcal{L}, \mathcal{L}^{i, e}$ are the elliptic operators

$$
\mathcal{L}^{i, e}: \zeta \in H^{1}(\Omega) \mapsto-\operatorname{div}\left(A^{i, e}(x) \nabla \zeta(x)\right), \quad \mathcal{L}:=\mathcal{L}^{i}+\mathcal{L}^{e},
$$

${ }^{\dagger}$ For simplicity, in the following we will keep fixed $p=2$ as before. 
$\boldsymbol{n}$ is the outer unit normal to $\partial \Omega$ and $\overline{\boldsymbol{n}}, \overline{\boldsymbol{n}}^{i, e}$ are the associated conormal vectors on $\partial \Omega$

$$
\overline{\boldsymbol{n}}^{i, e}:=A^{i, e} \boldsymbol{n}, \quad \overline{\boldsymbol{n}}:=\overline{\boldsymbol{n}}^{i}+\overline{\boldsymbol{n}}^{e} .
$$

It is interesting to know whether (1.40)-(1.42) hold also in the limit as $\varepsilon \downarrow 0$. An answer to this question is given in the following result, where, according to the previous convention, $q$ and $A$ denote the sum of $q^{i, e}$ and $A^{i, e}$ respectively.

THEOREM 1.5 There exists the $\Gamma\left(L^{2}(\Omega)\right)$-limit $G$ of $G^{\varepsilon}$, as $\varepsilon$ goes to $0 . G(u)$ is finite if and only if $u$ is a function of bounded variation in $\Omega$ assuming only the two values $s_{-}, s_{+}$a.e., and on this kind of function $G$ can be represented as in (1.24), i.e.

$$
G(u)=\int_{S_{u}^{*}} \phi\left(x, v_{u}(x)\right) \mathrm{d} H^{n-1}(x),
$$

where $\phi$ is defined by (1.25). Finally, if

$$
\Omega \text { is convex or of class } C^{1,1}, \quad A^{i, e} \text { are Lipschitz continuous }
$$

and

$$
u^{\varepsilon} \rightarrow u \in L^{2}(\Omega), \quad \underset{\varepsilon \downarrow 0}{\lim \sup } G^{\varepsilon}\left(u^{\varepsilon}\right) \leqslant G(u),
$$

then there exists $\boldsymbol{u}^{\varepsilon} \in \mathcal{U}\left(u^{\varepsilon}\right)$ such that $\boldsymbol{u}^{\varepsilon} \rightarrow \boldsymbol{u}$ in $L^{2}\left(\Omega ; \mathbb{R}^{2}\right)$, and $\boldsymbol{u}$ solves the following 'very weak' formulation of the elliptic problem (1.42)

$$
\begin{gathered}
\int_{\Omega} u^{i} \mathcal{L}(\zeta) \mathrm{d} x=\int_{\Omega} u \mathcal{L}^{e}(\zeta) \mathrm{d} x+\int_{\partial \Omega} u \partial_{\overline{\boldsymbol{n}}^{e} \zeta} \zeta \mathrm{d} H^{n-1}(x), \\
\int_{\Omega} u^{e} \mathcal{L}(\zeta) \mathrm{d} x=-\int_{\Omega} u \mathcal{L}^{i}(\zeta) \mathrm{d} x-\int_{\partial \Omega} u \partial_{\overline{\boldsymbol{n}}^{i}} \zeta \mathrm{d} H^{n-1}(x),
\end{gathered}
$$

for every test function $\zeta \in H^{2}(\Omega)$ with $\partial_{\overline{\boldsymbol{n}}} \zeta=0$ on $\partial \Omega$.

COROLlaRy 1.6 Let $\boldsymbol{u}^{\varepsilon} \in L^{2}\left(\Omega ; \mathbb{R}^{2}\right)$ such that

$$
\ell\left(\boldsymbol{u}^{\varepsilon}\right) \rightarrow u \quad \text { in } D^{\prime}(\Omega) \text { as } \varepsilon \downarrow 0 .
$$

Then

$$
\liminf _{\varepsilon \downarrow 0} F^{\varepsilon}\left(\boldsymbol{u}^{\varepsilon}\right) \geqslant G(u) .
$$

REMARK 1.7 Let us make a few comments about these results:

- The trace on $\partial \Omega$ of $u$ in the last integrals of $(1.47 \mathrm{a}, \mathrm{b})$ is well defined in the $B V$-sense and it belongs to $L^{\infty}(\partial \Omega)$.

- The solution $\boldsymbol{u}=\left(u^{i},-u^{e}\right)$ of $(1.47 \mathrm{a}, \mathrm{b})$, which is defined up to an additive constant $\boldsymbol{c}=$ $(c,-c)$, does not belong to $H^{1}\left(\Omega ; \mathbb{R}^{2}\right)$ in general; but if $\boldsymbol{u} \in H^{1}\left(\Omega ; \mathbb{R}^{2}\right)$ then it is also the usual variational solution of the system (cf. (4.4) below). 
- Corollary 1.6 follows Theorem 1.5 and the equicoercivity of $G^{\varepsilon}$.

- We assumed that the spatial dimension $n$ is equal to 3 for obvious physical reasons, but this hypothesis is not relevant for the validity of the mathematical results; on the contrary, the particular dimension $m=2$, which is related to the vectorial structure of the problem, plays an essential role in some proofs.

Thanks to the last items in the list on the $\Gamma$-limit of $F^{\varepsilon}$ and $G^{\varepsilon}$, we can show that, in general, the inequality (1.33) is strict. First of all we observe that the quadratic form $q$ defined by (1.30) is associated to the harmonic mean $\underline{A}$ of $A^{i}, A^{e}$ by the formulae (where we omit the explicit dependence on $x$ )

$$
\begin{gathered}
\underline{A}:=A^{i}\left(A^{i}+A^{e}\right)^{-1} A^{e}, \\
\underline{q}(\cdot, \xi)=\langle\underline{A} \xi, \xi\rangle .
\end{gathered}
$$

Analogously, $\bar{q}$ defined by (1.35) can be easily calculated by the 'harmonic mean' of the quadratic forms

$$
\bar{q}(x, \xi)=\frac{q^{i}(x, \xi) q^{e}(x, \xi)}{q^{i}(x, \xi)+q^{e}(x, \xi)} .
$$

It is obvious by the definition that $\phi \leqslant \bar{\phi}$, and it is not difficult to show that

$$
\underline{\phi}(x, \xi)=\bar{\phi}(x, \xi) \Leftrightarrow A^{i}(x) \xi, A^{e}(x) \xi \text { have the same direction. }
$$

If this is the case, of course, we have also

$$
\underline{\phi}(x, \xi)=\phi(x, \xi)=\bar{\phi}(x, \xi) .
$$

As we said before, in all the other cases we have a strict inequality.

THEOREM 1.8 If $A^{i}\left(x_{0}\right) \xi$ and $A^{e}\left(x_{0}\right) \xi$ have different directions, then

$$
\underline{\phi}\left(x_{0}, \xi\right)<\phi\left(x_{0}, \xi\right) \text {. }
$$

Remarks and open problems. Formal asymptotics in the bidomain case $[5,12,13]$, suggest that

$$
\phi(x, v)=\bar{\phi}(x, v)=\gamma_{0} \sqrt{\bar{q}(x, v)}
$$

where $\gamma_{0}$ and $\bar{q}$ are defined in (1.34) and (1.50) respectively; if this would be the case, (1.50) should provide an explicit and manageable formula to deal with the study, both theoretic and numeric, of the anisotropic curvature flow governed by the energy surface density $\phi$ (cf. [5]).

On the other hand, in some pathophysiological setting, such as regional ischaemia and heals infarction, the corresponding conductivity tensors $A^{i}, A^{e}$ do not yield a convex $\bar{\phi}$ : since $\phi$ is always convex (cf. Theorem 1.1), in this case (1.54) does not hold. It would be interesting to check if the convex envelope of $\bar{\phi}$ is a good substitute for (1.54) in this case. As we already said at the beginning of this introduction, we considered only the stationary problem; the rigorous justification of the evolution law still remains, to the best of our knowledge, an open problem. 
Plan of the paper We will collate in Section 2 some preliminary notation and results which will be useful in the remainder of this paper: some of them are well-known parts of deep and widely developed theories, which we recall here for the reader's convenience, without claiming any completeness. We will refer to the quoted books and papers for a systematic presentation of these subjects and for more detailed references.

Section 3, devoted to the proof of Theorems 1.1 and 1.2, has been divided into some subsections in order to make the logical connection of the arguments and the crucial technical points of the proof clearer.

In the last sections we will consider in more detail the case of the 'bidomain model': Theorem 1.5 is considered in Section 4 and the proof of the strict inequality of Theorem 1.8 is given in Section 5.

We remark that the intrinsic length of some of the proofs is justified by the attempt to develop a sufficiently abstract scheme, which could be directly applied to different applications. Moreover, many technical difficulties, related to the lack of coercivity, have often precluded a more direct appeal to already known results.

Finally, we notice that the continuity assumption on $\boldsymbol{q}(x, \cdot)$ (and therefore on the conductivity tensors $A^{i, e}$ ) can probably be relaxed, but we have kept it in order to avoid additional technical difficulties and complications in the representation formula for $\phi$.

\section{Notation and definitions}

\subsection{Preliminary notation}

From now on we fix a bounded, Lipschitz, and connected open set $\Omega \subset \mathbb{R}^{n}$. By $\boldsymbol{A}(\Omega)$ we denote the collection of the open subsets of $\Omega$. If $\mathcal{O}_{1}, \mathcal{O}_{2} \in A(\Omega)$, we say that $\mathcal{O}_{1} \Subset \mathcal{O}_{2}$ if $\overline{\mathcal{O}_{1}} \subset \mathcal{O}_{2}$. For a given set $U \subset \mathbb{R}^{n}$, we denote by $H^{n-1}(U)$ its $n-1$-dimensional Hausdorff measure and by $|U|$ its Lebesgue measure.

$\mathbb{S}^{n-1}$ is the set of unit vectors in $\mathbb{R}^{n}$. If $\mathcal{O} \subset \mathbb{R}^{n}$ is an open set, $w \in L_{\text {loc }}^{1}(\mathcal{O})$, and $\eta$ is a nonvanishing vector, we denote by $D_{\eta} w$ the distributional derivative of $w$ along $\eta$, which coincides with the classical one $\partial_{\eta} w=\langle\nabla w, \eta\rangle$ if $w \in C^{1}(\mathcal{O})$; we also use the symbol $\partial_{\eta} w$ if $D_{\eta} w \in L_{l o c}^{1}(\mathcal{O})$. $\partial_{1}, \ldots, \partial_{n}$ and $D_{1}, \ldots, D_{n}$ denote the derivatives along the directions of the canonical basis of $\mathbb{R}^{n}$.

We will deal with functionals depending on the continuous paramenter $\varepsilon>0$ or on the discrete values of a suitable decreasing infinitesimal sequence $\left\{\varepsilon_{n}\right\}_{n \in \mathbb{N}}$; for notational convenience, we will treat both the cases in the same way, by considering a general not empty set $E$ of real numbers such that

$$
E \subset(0,+\infty), \quad \text { inf } E=0 \text {. }
$$

Expressions like $\lim _{\varepsilon \downarrow 0, \varepsilon \in E}, \liminf _{\varepsilon \downarrow 0, \varepsilon \in E}$, etc ... have an obvious meaning as limits for $\varepsilon$ going to 0 in $E$. Of course, when $E$ contains an open interval $(0, \delta), \delta>0$, we will use the usual notation $\lim _{\varepsilon \downarrow}$. 


\subsection{Localization of functionals}

It will be useful to deal with the localized version of $F^{\varepsilon}, G^{\varepsilon}$ (and their $\Gamma$-limits). Therefore, for every $\mathcal{O} \in \boldsymbol{A}(\Omega), u \in L^{2}(\Omega)$, and $\boldsymbol{u} \in L^{2}\left(\Omega ; \mathbb{R}^{m}\right)$ we introduce

$$
\begin{gathered}
F^{\varepsilon}(\boldsymbol{u}, \mathcal{O}):= \begin{cases}\int_{\mathcal{O}} f^{\varepsilon}(x, \boldsymbol{u}, \nabla \boldsymbol{u}) \mathrm{d} x & \text { if } \boldsymbol{u} \in H^{1}\left(\mathcal{O} ; \mathbb{R}^{m}\right), \\
+\infty & \text { otherwise, }\end{cases} \\
Q(u, \mathcal{O}):=\min \left\{\int_{\mathcal{O}} \boldsymbol{q}(x, \nabla \boldsymbol{u}(x)) \mathrm{d} x: \boldsymbol{u} \in H^{1}\left(\mathcal{O} ; \mathbb{R}^{m}\right), \ell(\boldsymbol{u})=u \text { in } \mathcal{O}\right\}, \\
G^{\varepsilon}(u, \mathcal{O}):=\varepsilon Q(u, \mathcal{O})+\frac{1}{\varepsilon} \int_{\mathcal{O}} W(u(x)) \mathrm{d} x,
\end{gathered}
$$

where, as usual,

$$
H^{1}\left(\mathcal{O} ; \mathbb{R}^{m}\right):=\left\{\boldsymbol{u} \in L^{2}\left(\mathcal{O} ; \mathbb{R}^{m}\right): \partial_{i} \boldsymbol{u}=D_{i} \boldsymbol{u} \in L^{2}\left(\mathcal{O} ; \mathbb{R}^{m}\right), \quad i=1,2, \ldots, n\right\} .
$$

We observe that these functionals are local, lower semicontinuous, and increasing, in the sense of [16: Definition 1.1, 1.2; 15: Definition 15.1]: we collate here these and some other related definitions, which will be useful in the following section.

Let $X$ be a topological space included in $L_{l o c}^{1}\left(\Omega ; \mathbb{R}^{d}\right)$ and let $J$ be a functional defined on $X \times \boldsymbol{A}(\Omega)$ taking its values in the extended half line $[0,+\infty]$; we say that $J$ is

local if for every $\mathcal{O} \in \boldsymbol{A}(\Omega)$ and $u, w \in X$

$$
u_{\left.\right|_{\mathcal{O}}}=w_{\left.\right|_{\mathcal{O}}} \quad \Rightarrow \quad J(u, \mathcal{O})=J(w, \mathcal{O}),
$$

lower semicontinuous if for every $\mathcal{O} \in \boldsymbol{A}(\Omega)$,

$$
\text { the map } u \mapsto J(u, \mathcal{O}) \text { is lower semicontinuous in } X \text {, }
$$

increasing if for every $u \in X$ and $\mathcal{O}_{1}, \mathcal{O}_{2} \in A(\Omega)$

$$
\mathcal{O}_{1} \subset \mathcal{O}_{2} \quad \Rightarrow \quad J\left(u, \mathcal{O}_{1}\right) \leqslant J\left(u, \mathcal{O}_{2}\right),
$$

superadditive if for every $u \in X$ and $\mathcal{O}_{1}, \mathcal{O}_{2} \in A(\Omega)$

$$
\mathcal{O}_{1} \cap \mathcal{O}_{2} \neq \varnothing \quad \Rightarrow \quad J\left(u, \mathcal{O}_{1} \cup \mathcal{O}_{2}\right) \geqslant J\left(u, \mathcal{O}_{1}\right)+J\left(u, \mathcal{O}_{2}\right),
$$

weakly subadditive if for every $u \in X$ and $\mathcal{O}_{1}, \mathcal{O}_{1}^{\prime}, \mathcal{O}_{2} \in A(\Omega)$

$$
\mathcal{O}_{1} \Subset \mathcal{O}_{1}^{\prime} \quad \Rightarrow \quad J\left(u, \mathcal{O}_{1} \cup \mathcal{O}_{2}\right) \leqslant J\left(u, \mathcal{O}_{1}^{\prime}\right)+J\left(u, \mathcal{O}_{2}\right),
$$

inner regular if for every $u \in X$ and $\mathcal{O} \in \boldsymbol{A}(\Omega)$

$$
J(u, \mathcal{O})=\sup _{\mathcal{O}_{1} \Subset \mathcal{O}} J\left(u, \mathcal{O}_{1}\right),
$$


a measure if for every $u \in X$ the map $\mathcal{O} \mapsto J(u, \mathcal{O})$ is the restriction to $A(\Omega)$ of a regular Borel measure: in this case, the value of this measure on an arbitrary Borel set $B$ can be recovered by

$$
J(u, B):=\inf \{J(u, \mathcal{O}): \mathcal{O} \in A(\Omega), B \subset \mathcal{O}\} .
$$

An important result of [17: Proposition 5.5 and Theorem 5.6] (cf. also [15: Theorem 14.23]), ensures that $J$ is a measure iff $J$ is increasing, superadditive, weakly subadditive and inner regular.

Finally, we recall that to every increasing functional $J$ can be associated its inner regular envelope $J^{*}$, which is defined, for every $u \in X$ and $\mathcal{O} \in A(\Omega)$, by

$$
J^{*}(u, \mathcal{O}):=\sup \left\{J\left(u, \mathcal{O}_{1}\right): \mathcal{O}_{1} \in A(\Omega), \mathcal{O}_{1} \Subset \mathcal{O}\right\}
$$

\section{$2.3 \Gamma$-convergence}

(cf. [15: Definition 4.1, Proposition 8.1]) Let us fix a set $E$ as in (2.1), a topological space $X$ satisfying the first axiom of countability and a family $\left\{J^{\varepsilon}\right\}_{\varepsilon \in E}$ of functionals defined on $X$ with values in the extended real line $[-\infty,+\infty]$. The $\Gamma$-lower limit and the $\Gamma$-upper limit of $J^{\varepsilon}$, as $\varepsilon$ vanishes in $E$, are the (extended) real function on $X$ defined by

$$
\begin{aligned}
& \Gamma(X)-\liminf _{\varepsilon \downarrow 0, \varepsilon \in E} J^{\varepsilon}(u)=\inf \left\{\liminf _{\varepsilon \downarrow 0, \varepsilon \in E} J^{\varepsilon}\left(u^{\varepsilon}\right): \lim _{\varepsilon \downarrow 0, \varepsilon \in E} u^{\varepsilon}=u \quad \text { in } X\right\}
\end{aligned}
$$

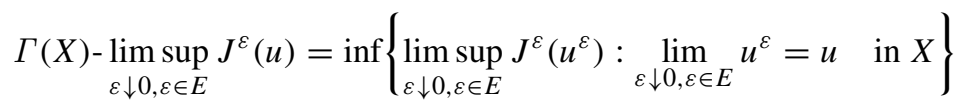

$$
\begin{aligned}
& \Gamma(X)-\liminf _{\varepsilon \downarrow 0, \varepsilon \in E} J^{\varepsilon}(u)=\Gamma(X)-\limsup _{\varepsilon \downarrow 0, \varepsilon \in E} J^{\varepsilon}(u), \quad \forall u \in X .
\end{aligned}
$$

We say that $J^{\varepsilon} \Gamma(X)$-converge as $\varepsilon \downarrow 0, \varepsilon \in E$, to the common value of both functionals, denoted by $\Gamma(X)-\lim _{\varepsilon \downarrow 0, \varepsilon \in E} J^{\varepsilon}$. When no misunderstanding is possible, we will omit the indication of $X$ and $E$ and we will use the symbols $J^{\prime}, J^{\prime \prime}$, and $J$ to denote the above $\Gamma$-limits. Since $X$ satisfies the first axiom of countability, when

$$
E \text { is the image of a decreasing infinitesimal sequence } n \mapsto \varepsilon_{n}>0 \text {, }
$$

the above definitions coincide with the general ones of [15: Definition 4.1]. Let us observe that $(2.5 \mathrm{a}, \mathrm{b})$ imply

$$
u^{\varepsilon} \rightarrow u \text { in } X \Rightarrow \liminf _{\varepsilon \downarrow 0, \varepsilon \in E} J^{\varepsilon}\left(u^{\varepsilon}\right) \geqslant J^{\prime}(u)
$$

and, for every $u \in X$,

$$
\exists\left\{u^{\varepsilon}\right\}_{\varepsilon \in E} \subset X: \quad \lim _{\varepsilon \downarrow 0, \varepsilon \in E} u^{\varepsilon}=u \text { in } X, \quad \limsup _{\varepsilon \downarrow 0, \varepsilon \in E} J^{\varepsilon}\left(u^{\varepsilon}\right) \leqslant J^{\prime \prime}(u) .
$$

Moreover, it follows immediately that if $D$ is a subset of $E$ with inf $D=0$ (in particular, if $D=$ $\left\{\varepsilon_{n}\right\}_{n \in \mathbb{N}}$ corresponds to a suitable subsequence in $E$ ) then

$$
\begin{aligned}
& \Gamma(X)-\liminf _{\varepsilon \downarrow 0, \varepsilon \in E} J^{\varepsilon} \leqslant \Gamma(X)-\liminf _{\varepsilon \downarrow 0, \varepsilon \in D} J^{\varepsilon} \\
\leqslant & \Gamma(X)-\limsup _{\varepsilon \downarrow 0, \varepsilon \in D} J^{\varepsilon} \leqslant \Gamma(X)-\limsup _{\varepsilon \downarrow 0, \varepsilon \in E} J^{\varepsilon} .
\end{aligned}
$$




\section{$2.4 \bar{\Gamma}$-convergence}

If $X$ is a topological space satisfying the first axiom of countability and $J^{\varepsilon}: X \times \boldsymbol{A}(\Omega) \rightarrow[0,+\infty]$, $\varepsilon \in E$, is a family of local increasing functionals (as considered in paragraph 2.2), for every open set $\mathcal{O}$ the functionals

$$
\begin{aligned}
J^{\prime}(\cdot, \mathcal{O}) & :=\Gamma(X)-\liminf _{\varepsilon \downarrow 0, \varepsilon \in E} J^{\varepsilon}(\cdot, \mathcal{O}), \\
J^{\prime \prime}(\cdot, \mathcal{O}) & :=\Gamma(X)-\limsup _{\varepsilon \downarrow 0, \varepsilon \in E} J^{\varepsilon}(\cdot, \mathcal{O})
\end{aligned}
$$

can be defined accordingly to $(2.5 \mathrm{a}, \mathrm{b})$ and are increasing and lower semicontinuous. If the inner regular envelopes of $J^{\prime}$ and $J^{\prime \prime}$ coincide, i.e. there exists an increasing, lower semicontinuous and inner regular functional $\bar{J}: X \times \boldsymbol{A}(\Omega)$ such that, for every $u \in X, \mathcal{O}_{1}, \mathcal{O}_{2} \in \boldsymbol{A}(\Omega)$

$$
\mathcal{O}_{1} \Subset \mathcal{O}_{2} \Rightarrow \bar{J}\left(u, \mathcal{O}_{1}\right) \leqslant J^{\prime}\left(u, \mathcal{O}_{1}\right) \leqslant J^{\prime \prime}\left(u, \mathcal{O}_{1}\right) \leqslant \bar{J}\left(u, \mathcal{O}_{2}\right),
$$

then we say that $J^{\varepsilon} \bar{\Gamma}(X)$-converge to $\bar{J}$ as $\varepsilon$ goes to 0 in $E$ [15: Definition 16.3, Proposition 16.4].

This notion of convergence for an increasing set functional has an important property, which holds if $X$ has a countable base of open sets [15: Proposition 16.8, Theorem 16.9]: a family $\left\{J^{\varepsilon}\right\}_{\varepsilon>0}$ $\bar{\Gamma}(X)$ converges to $\bar{J}$ as $\varepsilon \downarrow 0$ iff for every infinitesimal decreasing sequence $E=\left\{\varepsilon_{n}\right\}_{n \in \mathbb{N}}$

$$
\exists J_{E}=\bar{\Gamma}(X)-\lim _{\varepsilon \downarrow 0, \varepsilon \in E} J^{\varepsilon} \Rightarrow J_{E} \equiv \bar{J} ;
$$

in other words, in order to prove that $\left\{J^{\varepsilon}\right\}_{\varepsilon>0} \bar{\Gamma}(X)$ converges to $\bar{J}$ as $\varepsilon \downarrow 0$ it is sufficient to show that the $\Gamma$-limit $J_{E}$ on a subsequence $E$, whenever it exists, is independent of $E$.

\subsection{Functions of bounded variation}

$B V(\Omega)$ denotes the spaces of the functions $w \in L^{1}(\Omega)$ of bounded variation, whose distributional derivatives $D_{1} w, \ldots, D_{n} w$ are finite Borel (signed) measures, i.e.

$$
\int_{\Omega} w(x) \partial_{i} \zeta(x) \mathrm{d} x=-\int_{\Omega} \zeta(x) \mathrm{d} D_{i} w(x), \quad \forall \zeta \in C_{0}^{1}(\Omega), \quad i=1, \ldots, n .
$$

We will denote by $\left|D_{i} w\right|$ their total variations in the sense of measures on $\Omega$ : if $\mathcal{O} \in \boldsymbol{A}(\Omega)$ we have

$$
\left|D_{i} w\right|(\mathcal{O})=\sup \left\{\int_{\mathcal{O}} w(x) \partial_{i} \zeta(x) \mathrm{d} x: \zeta \in C_{0}^{1}(\mathcal{O}),|\zeta| \leqslant 1\right\} .
$$

The distributional gradient $D w:=\left(D_{1} w, \ldots, D_{n} w\right)$ is therefore a Borel vector measure on $\Omega$ with

$$
|D w|(\mathcal{O})=\sup \left\{\int_{\mathcal{O}} w(x) \operatorname{div} \zeta(x) \mathrm{d} x: \zeta \in C_{0}^{1}(\mathcal{O} ; \mathbb{R} m),|\zeta| \leqslant 1\right\}<+\infty .
$$

We recall that

$$
w \in W^{1,1}(\Omega) \quad \Rightarrow \quad w \in B V(\Omega), \quad|D w|(\mathcal{O})=\int_{\mathcal{O}}|\nabla w(x)| \mathrm{d} x .
$$


We define the space $B V(\Omega ; T)$ as

$$
B V(\Omega ; T):=\left\{u \in B V(\Omega): u(x) \in\left\{s_{-}, s_{+}\right\} \text {for a.e. } x \in \Omega\right\} .
$$

If $w \in B V(\Omega ; T)$, its level sets $\left\{x \in \Omega: w(x)=s_{-}\right\},\left\{x \in \Omega: w(x)=s_{+}\right\}$are both of finite perimeter; their common reduced boundary $S_{w}^{*}$ is the set of point $x_{0}$ belonging to the support of $|D w|$, such that

$$
\exists \lim _{\rho \rightarrow 0^{+}} \frac{D w\left(B\left(x_{0}, \rho\right)\right)}{|D w|\left(B\left(x_{0}, \rho\right)\right)}=v_{w}\left(x_{0}\right), \quad \text { and } \quad\left|v_{w}\left(x_{0}\right)\right|=1 .
$$

The Borel map $x \in S_{w}^{*} \mapsto v_{w}(x)$ is called generalized inner normal to $\left\{x \in \Omega: w(x)=s_{+}\right\}$, and we know that the measure $|D w|$ is concentrated on $S_{w}^{*}$, i.e.

$$
|D w|\left(\Omega \backslash S_{w}^{*}\right)=0,
$$

and, for every $\zeta \in C_{0}^{0}\left(\Omega ; \mathbb{R}^{n}\right)$,

$$
\int_{\Omega}\langle\zeta(x), D w\rangle:=\sum_{i=1}^{n} \int_{\Omega} \zeta_{i}(x) \mathrm{d} D_{i}(x)=\int_{S_{w}^{*}}\left\langle\zeta(x), v_{w}(x)\right\rangle \mathrm{d} H^{n-1}(x) .
$$

Slicing Let $Q$ be an open cylinder as in $(1.20 \mathrm{a}, \mathrm{b})$ where $\pi=\pi_{v}$, and $w$ is a real function defined on $Q$; we consider the family of one-dimensional sections

$$
w_{x}: t \in(a, b) \mapsto w_{x}(t):=w(x+t \eta) \in \mathbb{R} .
$$

It is well known that

$$
w \in L^{1}(Q) \quad \Rightarrow \quad w_{x} \in L^{1}(a, b) \quad \text { for a.e. } x \in Q_{0}
$$

with

$$
\int_{Q} w(x) \mathrm{d} x=|\langle\eta, v\rangle| \int_{\Sigma}\left(\int_{a}^{b} w_{x}(t) \mathrm{d} t\right) \mathrm{d} x
$$

If $w \in W_{l o c}^{1,1}(Q)$ an analogous property holds for the integral of the partial derivative of $w$ along $\eta$ :

$$
w \in W_{l o c}^{1,1}(Q) \quad \Rightarrow \quad w_{x} \in W_{l o c}^{1,1}(a, b), \quad \text { for a.e. } x \in \Sigma,
$$

with

$$
\int_{Q}\left|\partial_{\eta} w(x)\right| \mathrm{d} x=|\langle\eta, v\rangle| \int_{\Sigma}\left(\int_{a}^{b}\left|w_{x}^{\prime}(t)\right| \mathrm{d} t\right) \mathrm{d} x
$$

If $w \in L_{l o c}^{1}(Q)$ and $D_{\eta} w$ is a finite Borel measure on $Q$, then the previous formula can be rewritten as

$$
\begin{gathered}
w_{x} \in B V_{l o c}(a, b), \quad \text { for a.e. } x \in \Sigma, \\
\left|D_{\eta} w\right|(Q)=|\langle\eta, v\rangle| \int_{\Sigma}\left|D_{t} w_{x}\right|(a, b) \mathrm{d} x .
\end{gathered}
$$


Traces $\quad$ The standard trace operator $w \in W^{1,1}(\Omega) \mapsto w_{\left.\right|_{\partial \Omega}} \in L^{1}(\partial \Omega)$ can also be extended to a linear bounded operator from $B V(\Omega)$ to $L^{1}(\partial \Omega)$. The following related estimation will turn out to be useful (the proof is an easy adaptation of [10: Claim 4.5, Theorem 1, Section 5.3]).

LEMma 2.1 Let $w_{n} \in W^{1,1}(\Omega)$ be a sequence such that

$$
w_{n} \rightarrow w \quad \text { in } L^{1}(\Omega), \quad \lim _{\rho \downarrow 0} \limsup _{n \uparrow \infty} \int_{\Omega_{\rho}}\left|\nabla w_{n}(x)\right| \mathrm{d} x=0,
$$

where

$$
\Omega_{\rho}:=\{x \in \Omega: \mathrm{d}(x, \partial \Omega)<\rho\} .
$$

Then $\left.w_{n}\right|_{\partial \Omega}$ converges in $L^{1}(\partial \Omega)$ to the trace of $w$ on $\partial \Omega$.

If $Q$ has the cylindrical structure of $(1.20 \mathrm{a}, \mathrm{b})$, then we can combine traces and slicing: if $w \in$ $L_{l o c}^{1}(Q)$ and $D_{\eta} w$ is a bounded Borel measure, then

$$
\begin{gathered}
w_{x}(b)=w_{\left.\right|_{\Sigma_{+}}}(x), \quad w_{x}(a)=w_{\left.\right|_{\Sigma_{-}}}(x) \quad \text { a.e. in } \Sigma \\
|\langle\eta, v\rangle| \int_{\Sigma}\left|w_{x}(b)-w_{x}(a)\right| \mathrm{d} x \leqslant\left|D_{\eta} w\right|(Q) .
\end{gathered}
$$

\subsection{Integral representation of local functionals defined on $B V(\Omega ; T)$}

Let $J: L^{2}(\Omega) \times \boldsymbol{A}(\Omega) \rightarrow[0,+\infty]$ be a local, lower semicontinuous functional which is a measure in the sense of paragraph 2.2 and which is finite on $B V(\Omega ; T)$. It is interesting to know whether $J$ admits an integral representation like (1.19) for a suitable function $\phi$. The following result, which is a particular case of [9: Theorem 3.2; 2: Theorem 3.1], gives a very satisfactory answer to this problem. More precisely, if there exist positive constants $\lambda_{0}, \Lambda_{0}>0$ such that, $\forall u \in B V(\Omega ; T), \quad \forall \mathcal{O} \in \boldsymbol{A}(\Omega)$,

$$
\lambda_{0} H^{n-1}\left(S_{u}^{*} \cap \mathcal{O}\right) \leqslant J(u, \mathcal{O}) \leqslant \Lambda_{0} H^{n-1}\left(S_{u}^{*} \cap \mathcal{O}\right),
$$

then it is possible to find a uniquely determined (Borel) family of norms $\phi: \Omega \times \mathbb{R}^{n} \rightarrow[0,+\infty[$ such that

$$
J(u, \mathcal{O})=\int_{S_{u}^{*} \cap \mathcal{O}} \phi\left(x, v_{u}(x)\right) \mathrm{d} H^{n-1}(x), \quad \forall u \in B V(\Omega ; T), \quad \forall \mathcal{O} \in A(\Omega) .
$$

Moreover, if there exists a continuous modulus of continuity $\omega:[0,+\infty[\rightarrow[0,+\infty[$ such that $\omega(0)=0$ and

$$
|J(u, \mathcal{O})-J(v, \mathcal{O}+z)| \leqslant \omega(|z|) H^{n-1}\left(\mathcal{O} \cap S_{u}^{*}\right),
$$

whenever $\mathcal{O} \subset \Omega, z \in \mathbb{R}^{n},|z|<\frac{1}{2} \mathrm{~d}(\mathcal{O}, \partial \Omega)$ and $v(x+z)=u(x)$ in $\mathcal{O}$, then the seminorms $\phi(x, \cdot)$ are also continuous w.r.t. $x \in \Omega$. 


\subsection{A variant of the dominated convergence theorem}

[10: p. 22] Let $v, w,\left\{v_{n}, w_{n}\right\}_{n=1}^{+\infty}$ be measurable real functions defined in $\mathcal{O} \subset \mathbb{R}^{n}$ such that

$$
\left|v_{n}(x)\right|^{p} \leqslant w_{n}(x) \quad \text { for a.e. } x \in \mathcal{O}, \quad \forall n \in \mathbb{N}, \quad 1 \leqslant p<+\infty,
$$

and

$$
\begin{gathered}
\lim _{n \rightarrow+\infty} v_{n}(x)=v(x), \quad \lim _{n \rightarrow+\infty} w_{n}(x)=w(x) \text { a.e. in } \mathcal{O}, \\
\lim _{n \rightarrow+\infty} \int_{\mathcal{O}} w_{n}(x) \mathrm{d} x=\int_{\mathcal{O}} w(x) \mathrm{d} x<+\infty .
\end{gathered}
$$

Then

$$
\lim _{n \rightarrow+\infty} \int_{\mathcal{O}}\left|v_{n}(x)-v(x)\right|^{p} \mathrm{~d} x=0 .
$$

A simple application of this theorem shows the following.

Lemma 2.2 Let $W: \mathbb{R} \rightarrow\left[0,+\infty\left[, p_{0} \geqslant 2\right.\right.$, as in (1.15b). Then the map

$$
v \in L^{p_{0}}(\mathcal{O}) \mapsto W(v) \in L^{1}(\mathcal{O}) \quad \text { is continuous. }
$$

Moreover, if $v,\left\{v_{n}\right\}_{n=1}^{+\infty}$ are measurable functions such that $v_{n} \rightarrow v$ in $L^{1}(\mathcal{O})$, then

$$
v_{n} \rightarrow v \text { in } L^{p_{0}}(\mathcal{O}) \Leftrightarrow \lim _{n \rightarrow+\infty} \int_{\mathcal{O}} W\left(v_{n}(x)\right) \mathrm{d} x=\int_{\mathcal{O}} W(v(x)) \mathrm{d} x .
$$

\section{Proof of Theorems 1.1 and 1.2}

Let us briefly explain the sketch of the proof, which we tried to divide into several steps, in order to follow a possible general strategy to deal with this kind of singular perturbation problem.

First of all, we 'embed' the original $\Gamma$-convergence problem stated in $\Omega$ into a family of similar problems depending also on the open sets $\mathcal{O} \in \boldsymbol{A}(\Omega)$ : it is the standard localization technique introduced in Section 2.2. For this set-dependent approach we study the $\Gamma$-limits $F^{\prime}, F^{\prime \prime}$ defined by

$$
F^{\prime}(\cdot, \mathcal{O}):=\Gamma(X)-\liminf _{\varepsilon \downarrow 0, \varepsilon \in E} F^{\varepsilon}(\cdot, \mathcal{O}), \quad F^{\prime \prime}(\cdot, \mathcal{O}):=\Gamma(X)-\limsup _{\varepsilon \downarrow 0, \varepsilon \in E} F^{\varepsilon}(\cdot, \mathcal{O})
$$

and the weaker notion of $\bar{\Gamma}$-convergence as defined in Section 2.4. We already observed that showing this kind of convergence is equivalent to showing that the $\bar{\Gamma}$-limit of subsequences, whenever it exists, is uniquely determined and does not depend on the particular choice of subsequence.

So, we shall deal with such a limit $F^{\prime}, F^{\prime \prime}$, and $\bar{F}(\boldsymbol{u}, \mathcal{O})$, and we will prove that

1. $F^{\prime}(\boldsymbol{u}, \cdot), F^{\prime \prime}(\boldsymbol{u}, \cdot)$ are finite iff $u$ belongs to $B V(\mathcal{O} ; T)$ : this step requires suitable lower and upper bounds for $F^{\varepsilon}$; 
2. $\bar{F}(\boldsymbol{u}, \cdot)$ is a measure, in the sense of Section 2.2 ; this property is the heart of our procedure and follows by a careful subadditivity estimate, which also plays a fundamental role in the steps 5 and 6 below.

3. $F^{\prime}(\cdot ; \mathcal{O}), F^{\prime \prime}(\cdot, \mathcal{O})$ depend only on $u=\ell(\boldsymbol{u})$ : here we employ a simple approximation lemma.

4. $\bar{F}(\boldsymbol{u}, \cdot)$ coincides with $F^{\prime}(\boldsymbol{u}, \cdot)$ and $F^{\prime \prime}(\boldsymbol{u}, \cdot)$ on Lipschitz subsets and, in particular, on $\Omega$.

5. $\bar{F}$ can be represented by an anisotropic integral functional on the discontinuity set of $u$, by the general results detailed in Section 2.6.

6. The surface integrand $\phi$ can be identified in a simple parallelepipedal geometry as the cost of a transition through a planar surface with suitable boundary conditions, and, in particular, it is independent of $E$.

In Section 3.7 we will (quickly) repeat the same procedure to deal with the weak topology of $L^{2}$ and we will give the sketch of the proof of Theorem 1.2.

We fix once and for all a not empty subset

$$
E \subset(0,+\infty), \quad \text { with } \inf E=0
$$

as in (2.1) and we tacitly assume that the parameter $\varepsilon$ varies in $E$. We will denote by $X$ the Hilbert space $L^{2}\left(\Omega ; \mathbb{R}^{m}\right)$ endowed with its strong topology.

\subsection{Lower and upper bounds for $F^{\prime}, F^{\prime \prime}$; compactness}

The following results are strictly related to Propositions 1.3 and 1.4.

Lemma 3.1 Let $\boldsymbol{u} \in X$ satisfy $F^{\prime}(\boldsymbol{u}, \mathcal{O})<+\infty$, with $\mathcal{O} \in \boldsymbol{A}(\Omega)$. Then the restriction to $\mathcal{O}$ of $u:=\ell(\boldsymbol{u})$ belongs to $B V(\mathcal{O} ; T)$, and

$$
F^{\prime}(\boldsymbol{u}, \mathcal{O}) \geqslant \int_{S_{u}^{*} \cap \mathcal{O}} \phi\left(x, v_{u}(x)\right) \mathrm{d} H^{n-1}(x) \geqslant \lambda_{0} H^{n-1}\left(S_{u}^{*} \cap \mathcal{O}\right),
$$

where $\phi$ is defined by (1.33) and $\lambda_{0}:=\gamma_{0}|\ell|^{-1} \sqrt{\lambda}$.

Proof. From (1.30), if $\ell(\boldsymbol{u})=u$ we get easily

$$
F^{\varepsilon}(\boldsymbol{u}, \mathcal{O}) \geqslant \underline{G}^{\varepsilon}(u, \mathcal{O}):=\int_{\mathcal{O}}\left(\varepsilon \underline{q}(x, \nabla u(x))+\frac{1}{\varepsilon} W(u(x))\right) \mathrm{d} x ;
$$

then we can apply [8: Theorem 3.5, Remark 3.6(a) and (b) p. 299] to get

$$
\Gamma\left(L^{2}(\Omega)\right)-\lim _{\varepsilon \downarrow 0} \underline{G}^{\varepsilon}(u, \mathcal{O})= \begin{cases}\int_{S_{u}^{*} \cap \mathcal{O}} \underline{\phi}\left(x, v_{u}(x)\right) \mathrm{d} H^{n-1}(x) & \text { if } u \in B V(\mathcal{O} ; T) \\ +\infty & \text { otherwise. }\end{cases}
$$

By the obvious monotonicity property of the $\Gamma$-limits and (1.31), we conclude.

The following well-known compactness result underlies the previous lower bound and justifies (1.41). Here the function

$$
Z(s):=2 \int_{0}^{s} \sqrt{W(\tau)} \mathrm{d} \tau
$$


plays a fundamental role. Let us remark that

$$
Z \text { is strictly monotone, continuous, with its inverse } Z^{-1} \text {; }
$$

moreover, there exist constants $c_{0}, c_{1}>0$ such that

$$
c_{0}\left(|s|^{p_{0} / 2+1}-1\right) \leqslant|Z(s)| \leqslant c_{1}\left(|s|^{p_{0} / 2+1}+1\right), \quad \text { with } \quad 2 \leqslant p_{0} / 2+1 \leqslant p_{0} .
$$

LEMMA 3.2 Let $\mathcal{O}$ be an open subset of $\Omega$ and $\left\{\boldsymbol{u}^{\varepsilon}\right\}_{\varepsilon \in E}$ be a family in $X$ such that

$$
M:=\liminf _{\varepsilon \downarrow 0, \varepsilon \in E} F^{\varepsilon}\left(\boldsymbol{u}^{\varepsilon}, \mathcal{O}\right)<+\infty ;
$$

then there exist an infinitesimal decreasing sequence $D \subset E$ and a function $u \in B V(\mathcal{O} ; T)$ such that, setting $u^{\varepsilon}:=\ell\left(\boldsymbol{u}^{\varepsilon}\right)$

$$
\lim _{\varepsilon \downarrow 0, \varepsilon \in D} F^{\varepsilon}\left(\boldsymbol{u}^{\varepsilon} ; \mathcal{O}\right)=M, \quad \lim _{\varepsilon \downarrow 0, \varepsilon \in D} u^{\varepsilon}=u \text { in } L^{p_{0}}(\mathcal{O}),
$$

where $p_{0}$ is the growth exponent of $W$ defined in (1.15b). In particular, if $\boldsymbol{u}^{\varepsilon} \rightarrow \boldsymbol{u}$ in $X$ then $u^{\varepsilon} \rightarrow \ell(\boldsymbol{u})$ in $L^{p_{0}}(\mathcal{O})$.

Proof. Let us first observe that, by (3.2), we get

$$
F^{\varepsilon}\left(\boldsymbol{u}^{\varepsilon}, \mathcal{O}\right) \geqslant \int_{\mathcal{O}} 2 \sqrt{W\left(u^{\varepsilon}(x)\right) \underline{q}\left(x, \nabla u^{\varepsilon}(x)\right)} \mathrm{d} x \geqslant \int_{\mathcal{O}} \sqrt{\underline{q}\left(x, \nabla z^{\varepsilon}(x)\right)} \mathrm{d} x,
$$

where $z^{\varepsilon}(x):=Z\left(u^{\varepsilon}(x)\right)$. Let $D^{\prime} \subset E$ be an infinitesimal decreasing sequence satisfying the first equation of (3.8); (1.31) entails

$$
\sqrt{\underline{q}(x, \nabla z(x))} \geqslant \frac{\sqrt{\lambda}}{|\ell|}|\nabla z(x)|
$$

and by (3.6) we deduce that

$$
z^{\varepsilon}=Z\left(u^{\varepsilon}\right), \quad \varepsilon \in D^{\prime}
$$

is bounded in $B V(\mathcal{O})$. By a well-known argument [27], we can find another infinitesimal decreasing subsequence $D \subset D^{\prime}$ such that

$$
\lim _{\varepsilon \downarrow 0, \varepsilon \in D} u^{\varepsilon}=u, \quad \lim _{\varepsilon \downarrow 0, \varepsilon \in D} z^{\varepsilon}=z \quad \text { a.e. and in } L^{1}(\mathcal{O}) .
$$

Since

$$
F^{\varepsilon}\left(\boldsymbol{u}^{\varepsilon}, \mathcal{O}\right) \geqslant \frac{1}{\varepsilon} \int_{\mathcal{O}} W\left(u^{\varepsilon}(x)\right) \mathrm{d} x
$$

we also deduce

$$
\lim _{\varepsilon \downarrow 0, \varepsilon \in D} \int_{\mathcal{O}} W\left(u^{\varepsilon}(x)\right) \mathrm{d} x=\int_{\mathcal{O}} W(u(x)) \mathrm{d} x=0 .
$$

Applying Lemma 2.2 we also get the strong convergence in $L^{p_{0}}(\mathcal{O})$. 
Corollary 3.3 Let $\mathcal{O} \in \boldsymbol{A}(\Omega)$ and let $\boldsymbol{u}^{\varepsilon} \in X$ with $F^{\varepsilon}\left(\boldsymbol{u}^{\varepsilon}, \mathcal{O}\right)<+\infty, \varepsilon \in E$ satisfy

$$
u^{\varepsilon}:=\ell\left(\boldsymbol{u}^{\varepsilon}\right) \rightarrow u \in L^{2}(\mathcal{O}), \quad \liminf _{\varepsilon \downarrow 0, \varepsilon \in E} F^{\varepsilon}\left(\boldsymbol{u}^{\varepsilon}, \mathcal{O}\right)<+\infty .
$$

Then $u^{\varepsilon}, u \in L^{p_{0}}(\mathcal{O})$ and

$$
\lim _{\varepsilon \downarrow 0} \frac{\left\|u^{\varepsilon}-u\right\|_{L^{p_{0}}(\mathcal{O})}}{1+F^{\varepsilon}\left(\boldsymbol{u}^{\varepsilon} ; \mathcal{O}\right)}=0 .
$$

LEMma 3.4 Let $u \in L^{2}(\Omega)$ satisfy $u_{\left.\right|_{\mathcal{O}}} \in B V(\mathcal{O} ; T)$ for a Lipschitz open subset $\mathcal{O} \subset \Omega$. Then there exists $\boldsymbol{u} \in X$ such that $\ell(\boldsymbol{u})=u$ and $F^{\prime \prime}(\boldsymbol{u}, \mathcal{O})<+\infty$ with

$$
F^{\prime \prime}(\boldsymbol{u}, \mathcal{O}) \leqslant \int_{S_{u}^{*} \cap \mathcal{O}} \bar{\phi}\left(x, v_{u}(x)\right) \mathrm{d} H^{n-1}(x) \leqslant \Lambda_{0} H^{n-1}\left(S_{u}^{*} \cap \mathcal{O}\right),
$$

where $\bar{\phi}$ is defined by (1.37) and $\Lambda_{0}:=\gamma_{0}|\ell|^{-1} \sqrt{\Lambda}$.

Proof. Let us fix a Lipschitz function

$$
\boldsymbol{\alpha}=\left(\alpha_{1}, \ldots, \alpha_{m}\right): \Omega \rightarrow \mathbb{R}^{m}, \quad \text { such that } \ell(\boldsymbol{\alpha}) \equiv 1,
$$

and for every function $v \in L^{2}(\Omega)$ let us define

$$
\boldsymbol{v}_{\boldsymbol{\alpha}}(x):=v(x) \boldsymbol{\alpha}(x)
$$

of course, $\left.v_{\left.\right|_{\mathcal{O}}} \in H^{1}(\mathcal{O}) \Rightarrow v_{\alpha}\right|_{\mathcal{O}} \in H^{1}\left(\mathcal{O} ; \mathbb{R}^{m}\right)$

$$
\nabla \boldsymbol{v}_{\boldsymbol{\alpha}}=\boldsymbol{\alpha} \otimes \nabla v+v \nabla \boldsymbol{\alpha} \in \mathbb{M}^{m \times n} \quad \text { cf. the footnote in Proposition 1.1. }
$$

Cauchy-Schwarz inequality (3.34) yields, for every $\delta>0$,

$$
\begin{aligned}
F^{\varepsilon}\left(\boldsymbol{v}_{\alpha}, \mathcal{O}\right) & =\int_{\mathcal{O}}\left(\varepsilon \boldsymbol{q}\left(x, \nabla \boldsymbol{v}_{\alpha}(x)\right)+\frac{1}{\varepsilon} W(v(x))\right) \mathrm{d} x \\
& =\int_{\mathcal{O}}\left(\varepsilon \boldsymbol{q}(x, \boldsymbol{\alpha}(x) \otimes \nabla v(x)+v(x) \nabla \boldsymbol{\alpha}(x))+\frac{1}{\varepsilon} W(v(x))\right) \mathrm{d} x \\
& \leqslant(1+\delta) G_{\boldsymbol{\alpha}}^{\varepsilon}(v, \mathcal{O})+\left(1+\delta^{-1}\right) \varepsilon \Lambda\|\nabla \boldsymbol{\alpha}\|_{L^{\infty}\left(\Omega ; \mathbb{M}^{m \times n}\right)}^{2}\|v\|_{L^{2}(\mathcal{O})}^{2},
\end{aligned}
$$

where we defined

$$
G_{\boldsymbol{\alpha}}^{\varepsilon}(v, \mathcal{O}):=\int_{\mathcal{O}}\left(\varepsilon \boldsymbol{q}(x, \boldsymbol{\alpha}(x) \otimes \nabla v(x))+\frac{1}{\varepsilon} W(v(x))\right) \mathrm{d} x .
$$

Now we choose $\boldsymbol{u} \in L^{2}\left(\Omega ; \mathbb{R}^{m}\right)$ such that the restriction of $u:=\ell(\boldsymbol{u})$ to $\mathcal{O}$ belongs to $B V(\mathcal{O} ; T)$; by [8: Theorem 3.5, Remark 3.6(a) and (b) p. 299] we can find $u^{\varepsilon} \in L^{2}(\Omega)$ such that, as $\varepsilon \downarrow 0$,

$$
u^{\varepsilon} \rightarrow u \quad \text { in } L^{2}(\Omega), \quad G_{\alpha}^{\varepsilon}\left(u^{\varepsilon}, \mathcal{O}\right) \rightarrow \int_{S_{u}^{*} \cap \mathcal{O}} \phi_{\alpha}\left(x, v_{u}(x)\right) \mathrm{d} H^{n-1}(x)
$$


where

$$
\phi_{\alpha}(x, \xi):=\gamma_{0}(\boldsymbol{q}(x, \boldsymbol{\alpha}(x) \otimes \xi))^{1 / 2}
$$

We observe that

$$
\lim _{\varepsilon \downarrow 0} \boldsymbol{u}_{\boldsymbol{\alpha}}^{\varepsilon}=u \boldsymbol{\alpha} \quad \text { in } L^{2}\left(\Omega ; \mathbb{R}^{m}\right) \quad \text { and } \quad \ell(u \boldsymbol{\alpha})=u=\ell(\boldsymbol{u}) \quad \text { a.e. in } \mathcal{O}
$$

so that, since $\left\|u^{\varepsilon}\right\|_{L^{2}(\Omega)}$ are uniformly bounded, by (3.13) we get

$$
F^{\prime \prime}(u \boldsymbol{\alpha}, \mathcal{O}) \leqslant \limsup _{\varepsilon \downarrow 0} G_{\alpha}^{\varepsilon}\left(u^{\varepsilon}, \mathcal{O}\right) \leqslant(1+\delta) \int_{S_{u}^{*} \cap \mathcal{O}} \phi_{\alpha}\left(x, v_{u}(x)\right) \mathrm{d} H^{n-1}(x),
$$

and finally

$$
F^{\prime \prime}(u \boldsymbol{\alpha}, \mathcal{O}) \leqslant \int_{S_{u}^{*} \cap \mathcal{O}} \phi_{\alpha}\left(x, v_{u}(x)\right) \mathrm{d} H^{n-1}(x) .
$$

In this formula, we are free to choose $\alpha$ among the $\mathbb{R}^{m}$-valued Lipschitz real functions of $\Omega$, with the constraint $\ell(\boldsymbol{\alpha})=1$. The uniform estimate

$$
\begin{aligned}
\left|\phi_{\boldsymbol{\alpha}_{1}}(x, \xi)-\phi_{\boldsymbol{\alpha}_{2}}(x, \xi)\right| & \leqslant \sqrt{\boldsymbol{q}\left(x,\left(\boldsymbol{\alpha}_{1}(x)-\boldsymbol{\alpha}_{2}(x)\right) \otimes \xi\right)} \\
& \leqslant \sqrt{\Lambda}\left|\boldsymbol{\alpha}_{1}(x)-\boldsymbol{\alpha}_{2}(x)\right|, \quad \forall x \in \Omega, \xi \in \mathbb{S}^{n-1}
\end{aligned}
$$

the $X$-lower semicontinuity of $F^{\prime \prime}$, and a standard approximation argument ensure that we can also choose every $\boldsymbol{\alpha} \in C^{0}\left(\bar{\Omega} ; \mathbb{R}^{m}\right)$. Finally, an application of Lusin's Theorem to $\Omega$ endowed with the bounded Borel measure

$$
\mu(B):=H^{n-1}\left(B \cap S_{u}^{*} \cap \mathcal{O}\right)+|B|
$$

allows the choice of an arbitrary bounded Borel function $\boldsymbol{\alpha}: \Omega \rightarrow \mathbb{R}^{m}$. We choose $\boldsymbol{u}:=u \boldsymbol{\alpha}$ and $\boldsymbol{\alpha}$ such that

$$
\boldsymbol{q}\left(x, \boldsymbol{\alpha} \otimes v_{u}(x)\right)=\bar{q}\left(x, v_{u}(x)\right) \quad \forall x \in S_{u}^{*} \cap \mathcal{O} .
$$

\subsection{Additivity properties}

In this section we want to study some natural properties of the functionals $\mathcal{O} \in \boldsymbol{A}(\Omega) \mapsto$ $F^{\prime}(\boldsymbol{u}, \mathcal{O}), F^{\prime \prime}(\boldsymbol{u}, \mathcal{O})$, as defined in (3.13) for a fixed $\boldsymbol{u} \in X$. The first one is very simple, and follows by the general theory of [15: Proposition 16.12].

Lemma 3.5 For every $\boldsymbol{u} \in X$ the map $\mathcal{O} \mapsto F^{\prime}(\boldsymbol{u}, \mathcal{O})$ is superadditve, i.e. $\forall \mathcal{O}_{1}, \mathcal{O}_{2} \in \boldsymbol{A}(\Omega)$

$$
\mathcal{O}_{1} \cap \mathcal{O}_{2}=\varnothing \quad \Rightarrow \quad F^{\prime}\left(\boldsymbol{u}, \mathcal{O}_{1} \cup \mathcal{O}_{2}\right) \geqslant F^{\prime}\left(\boldsymbol{u}, \mathcal{O}_{1}\right)+F^{\prime}\left(\boldsymbol{u}, \mathcal{O}_{2}\right)
$$


The subadditivity of $F^{\prime \prime}$ is much more delicate to prove, even in the weak form introduced in paragraph 2.2. The main calculations, which are a careful modification of the techniques introduced by [16: Appendix] and [4: Lemma 3.2, Step 2], are collated in the following lemma.

Lemma 3.6 Let $\mathcal{O}_{1}, \mathcal{O}_{1}^{\prime}, \mathcal{O}_{2}$ be open subsets of $\Omega$ with $\mathcal{O}_{1} \Subset \mathcal{O}_{1}^{\prime}$, and suppose we are given two families $\boldsymbol{u}^{\varepsilon}, \boldsymbol{v}^{\varepsilon} \in L^{2}\left(\Omega ; \mathbb{R}^{m}\right), \varepsilon \in E$, such that

$$
\begin{aligned}
& \int_{\mathcal{O}_{1}^{\prime}}\left|\boldsymbol{u}^{\varepsilon}(x)\right|^{2} \mathrm{~d} x+\int_{\mathcal{O}_{2}}\left|\boldsymbol{v}^{\varepsilon}(x)\right|^{2} \mathrm{~d} x \leqslant C \\
u^{\varepsilon}:=\ell\left(\boldsymbol{u}^{\varepsilon}\right) \rightarrow u & \text { in } L^{2}\left(\mathcal{O}_{1}^{\prime}\right), \quad u \equiv v \text { in } \mathcal{O}_{1}^{\prime} \cap \mathcal{O}_{2} . \\
v^{\varepsilon}:= & \ell\left(\boldsymbol{v}^{\varepsilon}\right) \rightarrow v \quad \text { in } L^{2}\left(\mathcal{O}_{2}\right)
\end{aligned}
$$

Then there exist $\boldsymbol{w}^{\varepsilon} \in L^{2}\left(\Omega ; \mathbb{R}^{m}\right)$ which are a pointwise convex combination of $\boldsymbol{u}^{\varepsilon}$ and $\boldsymbol{v}^{\varepsilon}$ such that

$$
\begin{array}{ll}
\boldsymbol{w}^{\varepsilon} \equiv \boldsymbol{u}^{\varepsilon} & \text { in } \mathcal{O}_{1}, \\
\boldsymbol{w}^{\varepsilon} \equiv \boldsymbol{v}^{\varepsilon} & \text { in } \mathcal{O}_{2} \backslash \mathcal{O}_{1}^{\prime}, \quad \lim _{\varepsilon \downarrow 0, \varepsilon \in E} \ell\left(\boldsymbol{w}^{\varepsilon}\right)=u \quad \text { in } L^{2}\left(\mathcal{O}_{1}^{\prime} \cap \mathcal{O}_{2}\right),
\end{array}
$$

and

$$
\begin{aligned}
& \limsup _{\varepsilon \downarrow 0, \varepsilon \in E} F^{\varepsilon}\left(\boldsymbol{w}^{\varepsilon}, \mathcal{O}_{1} \cup \mathcal{O}_{2}\right) \leqslant \limsup _{\varepsilon \downarrow 0, \varepsilon \in E}\left(F^{\varepsilon}\left(\boldsymbol{u}^{\varepsilon}, \mathcal{O}_{1}^{\prime}\right)+F^{\varepsilon}\left(\boldsymbol{v}^{\varepsilon}, \mathcal{O}_{2}\right)\right) \\
& \liminf _{\varepsilon \downarrow 0, \varepsilon \in E} F^{\varepsilon}\left(\boldsymbol{w}^{\varepsilon}, \mathcal{O}_{1} \cup \mathcal{O}_{2}\right) \leqslant \liminf _{\varepsilon \downarrow 0, \varepsilon \in E}\left(F^{\varepsilon}\left(\boldsymbol{u}^{\varepsilon}, \mathcal{O}_{1}^{\prime}\right)+F^{\varepsilon}\left(\boldsymbol{v}^{\varepsilon}, \mathcal{O}_{2}\right)\right) .
\end{aligned}
$$

Moreover, if

$$
\begin{array}{ll}
\boldsymbol{u}^{\varepsilon}-\boldsymbol{u} & \text { in } L^{2}\left(\mathcal{O}_{1}^{\prime} ; \mathbb{R}^{m}\right), \\
\boldsymbol{v}^{\varepsilon}-\boldsymbol{v} & \text { in } L^{2}\left(\mathcal{O}_{2} ; \mathbb{R}^{m}\right),
\end{array} \quad \boldsymbol{u} \equiv \boldsymbol{v} \quad \text { in } \mathcal{O}_{1}^{\prime} \cap \mathcal{O}_{2},
$$

then we can also ask that

$$
\boldsymbol{w}^{\varepsilon} \rightarrow \boldsymbol{w} \in L^{2}\left(\Omega ; \mathbb{R}^{m}\right), \quad \boldsymbol{w}=\boldsymbol{u} \quad \text { in } \mathcal{O}_{1}^{\prime}, \quad \boldsymbol{w}=\boldsymbol{v} \quad \text { in } \mathcal{O}_{2} .
$$

REMARK 3.7 If the convergences in (3.20) are strong, i.e.

$$
\begin{array}{ll}
\boldsymbol{u}^{\varepsilon} \rightarrow \boldsymbol{u} & \text { in } L^{2}\left(\mathcal{O}_{1}^{\prime} ; \mathbb{R}^{m}\right), \quad \boldsymbol{u} \equiv \boldsymbol{v} \quad \text { in } \mathcal{O}_{1}^{\prime} \cap \mathcal{O}_{2}, \\
\boldsymbol{v}^{\varepsilon} \rightarrow \boldsymbol{v} & \text { in } L^{2}\left(\mathcal{O}_{2} ; \mathbb{R}^{m}\right),
\end{array}
$$

then it is easy to see that (3.21) also holds in the stronger form

$$
\boldsymbol{w}^{\varepsilon} \rightarrow \boldsymbol{w} \in L^{2}\left(\Omega ; \mathbb{R}^{m}\right), \quad \boldsymbol{w}=\boldsymbol{u} \quad \text { in } \mathcal{O}_{1}^{\prime}, \quad \boldsymbol{w}=\boldsymbol{v} \quad \text { in } \mathcal{O}_{2},
$$

since, by construction, $\boldsymbol{w}^{\varepsilon}$ is a pointwise convex combination of $\boldsymbol{u}^{\varepsilon}$ and $\boldsymbol{v}^{\varepsilon}$.

Proof. First of all, we observe that the interesting case is $\mathcal{O}_{1} \cap \mathcal{O}_{2} \neq \varnothing$; we denote by $g^{\varepsilon}, h^{\varepsilon}$ the sums

$$
\begin{gathered}
g^{\varepsilon}:=F^{\varepsilon}\left(\boldsymbol{u}^{\varepsilon}, \mathcal{O}_{1}^{\prime}\right)+F^{\varepsilon}\left(\boldsymbol{v}^{\varepsilon}, \mathcal{O}_{2}\right), \\
h^{\varepsilon}:=\left\|u^{\varepsilon}-u\right\|_{L^{p_{0}}\left(\mathcal{O}_{1}^{\prime}\right)}+\left\|v^{\varepsilon}-v\right\|_{L^{p_{0}}\left(\mathcal{O}_{2}\right)}
\end{gathered}
$$


and we know by Corollary 3.3 that

$$
\sigma^{\varepsilon}:=\frac{h^{\varepsilon}}{1+g^{\varepsilon}} \quad \text { vanishes as } \varepsilon \downarrow 0 .
$$

Moreover, it is not restrictive to assume that $\liminf _{\varepsilon \downarrow 0, \varepsilon \in E} g^{\varepsilon}<+\infty$ and $g^{\varepsilon}<+\infty$ for every diff in.pdf $\varepsilon \in E ;$ in particular, we can suppose that $\boldsymbol{u}^{\varepsilon} \in H^{1}\left(\mathcal{O}_{1}^{\prime} ; \mathbb{R}^{m}\right)$ and $\boldsymbol{v}^{\varepsilon} \in H^{1}\left(\mathcal{O}_{2} ; \mathbb{R}^{m}\right)$. We know that

$$
\int_{\mathcal{O}_{1}^{\prime} \cup \mathcal{O}_{2}} W(w(x)) \mathrm{d} x=0, \quad \text { where } \quad w \equiv u \text { in } \mathcal{O}_{1}^{\prime}, \quad w \equiv v \text { in } \mathcal{O}_{2} ;
$$

so that, introducing the modulus of continuity

$$
\rho(s):=\sup \left\{\int_{\mathcal{O}_{1}^{\prime} \cap \mathcal{O}_{2}} W(z(x)) \mathrm{d} x:\|z-w\|_{L^{p_{0}}\left(\mathcal{O}_{1}^{\prime} \cap \mathcal{O}_{2}\right)} \leqslant s\right\},
$$

By the result quoted in paragraph 2.5, we know that $\lim _{s \downarrow 0} \rho(s)=0$.

Let $\mathcal{Q}$ be a fixed Lipschitz open set, such that

$$
\mathcal{O}_{1} \subset \mathcal{Q} \Subset \mathcal{O}_{1}^{\prime},
$$

and let us fix $\varepsilon \in E$ and a positive integer $k$. As in [16: p. 216], let

$$
d:=\frac{1}{2} d\left(\mathcal{Q}, \partial \mathcal{O}_{1}^{\prime}\right)>0
$$

be the half-distance between $\mathcal{Q}$ and the boundary of $\mathcal{O}_{1}^{\prime}$, let us choose other $k$ open sets

$$
\mathcal{Q}=\mathcal{Q}_{0} \Subset \mathcal{Q}_{1} \Subset \cdots \Subset \mathcal{Q}_{k} \Subset \mathcal{O}_{1}^{\prime}
$$

separated from each other by a distance of width $r:=d / k$, and $k$ Lipschitz real functions

$$
\alpha_{1}, \ldots, \alpha_{k}: \Omega \rightarrow[0,1],
$$

in the following way:

$$
\begin{gathered}
\mathcal{Q}_{j}:=\{x \in \Omega: d(x, \partial \mathcal{Q})<j r\}, \\
\left.\alpha_{j}\right|_{\mathcal{Q}_{j-1}} \equiv 1,\left.\quad \alpha_{j}\right|_{\Omega \backslash \mathcal{Q}_{j}} \equiv 0,\left.\quad \alpha_{j}\right|_{\mathcal{Q}_{j} \backslash \mathcal{Q}_{j-1}}(x)=j-d(x, \mathcal{Q}) / r
\end{gathered}
$$

In particular

$$
\left\|\nabla \alpha_{j}\right\|_{L^{\infty}(\Omega)} \leqslant r^{-1}=k / d, \quad \int_{\Omega}\left|\nabla \alpha_{j}(x)\right| \mathrm{d} x \leqslant r^{-1}\left|\mathcal{Q}_{j+1} \backslash \mathcal{Q}_{j}\right| \leqslant C_{\mathcal{O}_{1}}
$$

where $C_{\mathcal{O}_{1}}$ is a constant depending on the Lipschitz character of $\mathcal{O}_{1}$. We define

$$
\boldsymbol{w}_{j}(x):=\alpha_{j}(x) \boldsymbol{u}^{\varepsilon}(x)+\left(1-\alpha_{j}(x)\right) \boldsymbol{v}^{\varepsilon}(x), \quad j=1, \ldots, k,
$$

\footnotetext{
Otherwise we set

$\boldsymbol{w}^{\varepsilon} \equiv \boldsymbol{u}^{\varepsilon} \quad$ in $\mathcal{O}_{1}, \quad \boldsymbol{w}^{\varepsilon} \equiv \boldsymbol{v}^{\varepsilon} \quad$ in $\Omega \backslash \mathcal{O}_{1}, \quad$ if $g^{\varepsilon}=+\infty$
}

and we restrict our attention to $E^{\prime}:=\left\{\varepsilon \in E: g^{\varepsilon}<+\infty\right\}$. 
and we want to estimate $F^{\varepsilon}\left(\boldsymbol{w}_{j}, \mathcal{O}_{1} \cup \mathcal{O}_{2}\right)$. We consider the partition covering $\mathcal{O}_{1} \cup \mathcal{O}_{2}$ given by

$$
\mathcal{Q}_{j}, \mathcal{O}_{2} \cap\left(\mathcal{Q}_{j+1} \backslash \mathcal{Q}_{j}\right), \mathcal{O}_{2} \backslash \mathcal{Q}_{j+1}
$$

and we observe that $\boldsymbol{w}_{j} \in H^{1}\left(\mathcal{O}_{1}^{\prime} \cap \mathcal{O}_{2}\right)$,

$$
\boldsymbol{w}_{j} \equiv \boldsymbol{u}^{\varepsilon} \quad \text { on } \mathcal{Q}_{j} \supset \mathcal{O}_{1}, \quad \boldsymbol{w}_{j} \equiv \boldsymbol{v}^{\varepsilon} \quad \text { on } \mathcal{O}_{2} \backslash \mathcal{Q}_{j+1}=\mathcal{O}_{2} \backslash \mathcal{Q}_{j+1}
$$

and

$$
\nabla \boldsymbol{w}_{j}=\alpha_{j} \nabla \boldsymbol{u}^{\varepsilon}+\left(1-\alpha_{j}\right) \nabla \boldsymbol{v}^{\varepsilon}+\nabla \alpha_{j}\left(\boldsymbol{u}^{\varepsilon}-\boldsymbol{v}^{\varepsilon}\right), \quad \text { in } \mathcal{O}_{2} \cap\left(\mathcal{Q}_{j+1} \backslash \mathcal{Q}_{j}\right) .
$$

By the convexity of $\boldsymbol{q}$ and the Cauchy inequality (3.34), for every $\delta>0$ and a.e. $x \in \mathcal{O}_{1}^{\prime} \cap \mathcal{O}_{2}$ we get

$$
\begin{aligned}
\boldsymbol{q}\left(x, \nabla \boldsymbol{w}_{j}\right) & \leqslant(1+\delta)\left[\alpha_{j} \boldsymbol{q}\left(x, \nabla \boldsymbol{u}^{\varepsilon}\right)+\left(1-\alpha_{j}\right) \boldsymbol{q}\left(x, \nabla \boldsymbol{v}^{\varepsilon}\right)\right] \\
& +\Lambda\left(1+\delta^{-1}\right) \frac{k^{2}}{d^{2}}\left|\boldsymbol{u}^{\varepsilon}-\boldsymbol{v}^{\varepsilon}\right|^{2}
\end{aligned}
$$

and therefore

$$
\begin{aligned}
\int_{\mathcal{O}_{1} \cup \mathcal{O}_{2}} \boldsymbol{q}\left(x, \nabla \boldsymbol{w}_{j}\right) \mathrm{d} x & \leqslant(1+\delta)\left(\int_{\mathcal{O}_{1}^{\prime}} \boldsymbol{q}\left(x, \nabla \boldsymbol{u}^{\varepsilon}\right) \mathrm{d} x+\int_{\mathcal{O}_{2}} \boldsymbol{q}\left(x, \nabla \boldsymbol{v}^{\varepsilon}\right) \mathrm{d} x\right) \\
& +\Lambda \frac{\left(1+\delta^{-1}\right)}{d^{2}} k^{2} \int_{\mathcal{O}_{2} \cap\left(\mathcal{Q}_{j+1} \backslash \mathcal{Q}_{j}\right)}\left|\boldsymbol{u}^{\varepsilon}-\boldsymbol{v}^{\varepsilon}\right|^{2} \mathrm{~d} x
\end{aligned}
$$

On the other hand, we easily obtain

$$
\int_{\mathcal{O}_{1} \cup \mathcal{O}_{2}} \tilde{W}\left(\boldsymbol{w}_{j}\right) \mathrm{d} x \leqslant \int_{\mathcal{O}_{1}^{\prime}} \tilde{W}\left(\boldsymbol{u}^{\varepsilon}\right) \mathrm{d} x+\int_{\mathcal{O}_{2}} \tilde{W}\left(\boldsymbol{v}^{\varepsilon}\right) \mathrm{d} x+\int_{\mathcal{O}_{2} \cap\left(\mathcal{Q}_{j+1} \backslash \mathcal{Q}_{j}\right)} \tilde{W}\left(\boldsymbol{w}_{j}\right) \mathrm{d} x .
$$

Summing up these contributions and calling $c_{0}:=\frac{\Lambda\left(1+\delta^{-1}\right)}{d^{2}}$, we obtain

$$
\begin{aligned}
F^{\varepsilon}\left(\boldsymbol{w}_{j}, \mathcal{O}_{1} \cup \mathcal{O}_{2}\right) & \leqslant(1+\delta)\left(F^{\varepsilon}\left(\boldsymbol{u}^{\varepsilon}, \mathcal{O}_{1}^{\prime}\right)+F^{\varepsilon}\left(\boldsymbol{v}^{\varepsilon}, \mathcal{O}_{2}\right)\right) \\
+ & \int_{\mathcal{O}_{2} \cap\left(\mathcal{Q}_{j+1} \backslash \mathcal{Q}_{j}\right)}\left(c_{0} \varepsilon k^{2}\left|\boldsymbol{u}^{\varepsilon}-\boldsymbol{v}^{\varepsilon}\right|^{2}+\frac{1}{\varepsilon} \tilde{W}\left(\boldsymbol{w}_{j}\right)\right) \mathrm{d} x .
\end{aligned}
$$

Since $j$ can be arbitrarily chosen between 1 and $k$, we deduce that there exists $j^{*} \in\{1, \ldots, k\}$ such 
that

$$
\begin{aligned}
& F^{\varepsilon}\left(\boldsymbol{w}_{j^{*}}, \mathcal{O}_{1} \cup \mathcal{O}_{2}\right) \leqslant(1+\delta)\left(F^{\varepsilon}\left(\boldsymbol{u}^{\varepsilon}, \mathcal{O}_{1}^{\prime}\right)+F^{\varepsilon}\left(\boldsymbol{v}^{\varepsilon}, \mathcal{O}_{2}\right)\right) \\
& +\frac{1}{k} \sum_{j=1}^{k} \int_{\mathcal{O}_{2} \cap\left(\mathcal{Q}_{j+1} \backslash \mathcal{Q}_{j}\right)}\left(c_{0} \varepsilon k^{2}\left|\boldsymbol{u}^{\varepsilon}-\boldsymbol{v}^{\varepsilon}\right|^{2}+\frac{1}{\varepsilon} \tilde{W}\left(\boldsymbol{w}_{j}\right)\right) \mathrm{d} x \\
& \leqslant(1+\delta) g^{\varepsilon}+\int_{\mathcal{O}_{2} \cap \mathcal{O}_{1}^{\prime}}\left(c_{0} \varepsilon k\left|\boldsymbol{u}^{\varepsilon}-\boldsymbol{v}^{\varepsilon}\right|^{2}+\frac{1}{\varepsilon k} \tilde{W}\left(\boldsymbol{w}^{*}\right)\right) \mathrm{d} x \\
& \leqslant(1+\delta) g^{\varepsilon}+c_{1} \varepsilon k+\frac{1}{\varepsilon k} \int_{\mathcal{O}_{2} \cap \mathcal{O}_{1}^{\prime}} \tilde{W}\left(\boldsymbol{w}^{*}\right) \mathrm{d} x \\
& \leqslant(1+\delta) g^{\varepsilon}+c_{1} \varepsilon k+\frac{1}{\varepsilon k} \rho\left(\left\|w^{*}-u\right\|_{L^{p}\left(\mathcal{O}_{2} \cap \mathcal{O}_{1}^{\prime}\right)}\right),
\end{aligned}
$$

where we defined $c_{1}:=2 c_{0} C$ and $\boldsymbol{w}^{*}$ as

$$
\boldsymbol{w}^{*} \equiv \boldsymbol{w}_{j} \quad \text { on } \quad \mathcal{Q}_{j+1} \backslash \mathcal{Q}, \quad w^{*}=\ell\left(\boldsymbol{w}^{*}\right) .
$$

We observe that $w^{*}(x)$ is always a convex combination of $u^{\varepsilon}$ and $v^{\varepsilon}$, therefore

$$
\left\|w^{*}-w\right\|_{L^{p}\left(\mathcal{O}_{2} \cap \mathcal{O}_{1}^{\prime}\right)} \leqslant h^{\varepsilon} .
$$

Choosing $k$ as the integer part of $\varepsilon^{-1} \sqrt{\rho\left(\omega^{\varepsilon}\right)+\varepsilon}$ and $\boldsymbol{w}^{\varepsilon}:=\boldsymbol{w}_{j^{*}}$, we obtain (cf. (3.24))

$$
\begin{aligned}
F^{\varepsilon}\left(\boldsymbol{w}^{\varepsilon}, \mathcal{O}_{1} \cup \mathcal{O}_{2}\right) & \leqslant(1+\delta) g^{\varepsilon}+2 c_{1} \sqrt{\rho\left(h^{\varepsilon}\right)+\varepsilon} \\
& \leqslant(1+\delta) g^{\varepsilon}+2 c_{1} \sqrt{\rho\left(\sigma^{\varepsilon} g^{\varepsilon}\right)+\varepsilon} .
\end{aligned}
$$

Since $\sigma^{\varepsilon} \rightarrow 0$ as $\varepsilon \downarrow 0$, we deduce that

$$
\begin{aligned}
& \limsup _{\varepsilon \downarrow 0, \varepsilon \in E} F^{\varepsilon}\left(\boldsymbol{w}^{\varepsilon}, \mathcal{O}_{1} \cup \mathcal{O}_{2}\right) \leqslant(1+\delta) \limsup _{\varepsilon \downarrow 0, \varepsilon \in E} g^{\varepsilon} \\
& \liminf _{\varepsilon \downarrow 0, \varepsilon \in E} F^{\varepsilon}\left(\boldsymbol{w}^{\varepsilon}, \mathcal{O}_{1} \cup \mathcal{O}_{2}\right) \leqslant(1+\delta) \liminf _{\varepsilon \downarrow 0, \varepsilon \in E} g^{\varepsilon} .
\end{aligned}
$$

As $\delta>0$ is arbitrary, we obtain (3.18).

It remains to show that (3.20) entails (3.21). By the first two properties of (3.17), it is sufficient to prove the weak convergence of $\boldsymbol{w}^{\varepsilon}$ in $L^{2}\left(\mathcal{O}_{1}^{\prime} \cap \mathcal{O}_{2} ; \mathbb{R}^{m}\right)$. We will apply the result of the next lemma, where we define $\alpha^{\varepsilon}$ as the function $\alpha_{j^{*}}$ corresponding to the index $j^{*}$ introduced in the previous calculations.

Lemma 3.8 Let $\boldsymbol{u}^{\varepsilon}, \boldsymbol{v}^{\varepsilon}, \varepsilon \in E$, be two families weakly converging to the same limit $\boldsymbol{u}=\boldsymbol{v}$ in $L^{2}\left(\mathcal{O} ; \mathbb{R}^{m}\right)$, and let $\alpha^{\varepsilon}: \mathcal{O} \rightarrow[0,1]$ be a family of Lipschitz functions such that

$$
\int_{\mathcal{O}}\left|\nabla \alpha^{\varepsilon}(x)\right| \mathrm{d} x \leqslant A \quad \forall \varepsilon \in E .
$$

Then, setting

$$
\boldsymbol{w}^{\varepsilon}=\alpha^{\varepsilon} \boldsymbol{u}^{\varepsilon}+\left(1-\alpha^{\varepsilon}\right) \boldsymbol{v}^{\varepsilon},
$$

$\boldsymbol{w}^{\varepsilon}$ weakly converges to $\boldsymbol{w}=\boldsymbol{u}=\boldsymbol{v}$ in $L^{2}\left(\mathcal{O} ; \mathbb{R}^{m}\right)$. 
Proof. From (3.28) we get

$$
\boldsymbol{w}^{\varepsilon}-\boldsymbol{w}=\alpha^{\varepsilon}\left(\boldsymbol{u}^{\varepsilon}-\boldsymbol{u}\right)+\left(1-\alpha^{\varepsilon}\right)\left(\boldsymbol{v}^{\varepsilon}-\boldsymbol{v}\right) .
$$

Since $\boldsymbol{u}^{\varepsilon}-\boldsymbol{u} \rightarrow 0$ and $\boldsymbol{v}^{\varepsilon}-\boldsymbol{v} \rightarrow 0$ in $L^{2}\left(\mathcal{O} ; \mathbb{R}^{m}\right)$, our thesis follows if we show that for every test function $\zeta \in L^{2}(\mathcal{O})$ the family

$$
\{\alpha \zeta: \alpha: \mathcal{O} \rightarrow[0,1],|\nabla \alpha|(\mathcal{O}) \leqslant A\} \quad \text { is compact in } L^{2}(\mathcal{O}) .
$$

But this property is an easy consequence of the fact that every sequence of functions with values in $[0,1]$ and uniformly bounded total variation possesses an a.e. convergent subsequence.

Let us write some important consequences of Lemma 3.6 (cf. Section 2.2).

COROLLARY 3.9 The local functional $F^{\prime \prime}$ is weakly subadditive.

Proof. For a fixed $\boldsymbol{u} \in X$, choose $\boldsymbol{u}^{\varepsilon}, \boldsymbol{v}^{\varepsilon} \in X$ converging to $\boldsymbol{u}$ in $X$. Applying Lemma 3.6 we can find $\boldsymbol{w}^{\varepsilon} \in X$ converging to $\boldsymbol{u}$ such that

$$
\begin{aligned}
\limsup _{\varepsilon \downarrow 0, \varepsilon \in E} F^{\varepsilon}\left(\boldsymbol{w}^{\varepsilon}, \mathcal{O}_{1} \cup \mathcal{O}_{2}\right) & \leqslant \limsup _{\varepsilon \downarrow 0, \varepsilon \in E}\left(F^{\varepsilon}\left(\boldsymbol{u}^{\varepsilon}, \mathcal{O}_{1}^{\prime}\right)+F^{\varepsilon}\left(\boldsymbol{v}^{\varepsilon}, \mathcal{O}_{2}\right)\right) \\
& \leqslant \limsup _{\varepsilon \downarrow 0, \varepsilon \in E} F^{\varepsilon}\left(\boldsymbol{u}^{\varepsilon}, \mathcal{O}_{1}^{\prime}\right)+\limsup _{\varepsilon \downarrow 0, \varepsilon \in E} F^{\varepsilon}\left(\boldsymbol{v}^{\varepsilon}, \mathcal{O}_{2}\right) .
\end{aligned}
$$

It follows immediately that

$$
F^{\prime \prime}\left(\boldsymbol{u}, \mathcal{O}_{1} \cup \mathcal{O}_{2}\right) \leqslant F^{\prime \prime}\left(\boldsymbol{u}, \mathcal{O}_{1}^{\prime}\right)+F^{\prime \prime}\left(\boldsymbol{u}, \mathcal{O}_{2}\right) .
$$

Combining Corollary 3.9 and Lemma 3.5 we prove the third step described at the beginning of this section.

Corollary 3.10 Let us assume that there exists the $\bar{\Gamma}(X)$-limit (as defined in Section 2.4)

$$
\bar{F}=\bar{\Gamma}(X)-\lim _{\varepsilon \downarrow 0, \varepsilon \in E} F^{\varepsilon} .
$$

Then $\bar{F}$ is a measure in the sense of Section (2.2).

\subsection{The $\Gamma$-limits depend only on $u=\ell(\boldsymbol{u})$}

Now we want to show that the $\Gamma$-limits $F^{\prime}, F^{\prime \prime}$ (and therefore $\bar{F}$ ) depend only on $\ell(\boldsymbol{u}$ ). This fact follows by the following simple approximation lemma.

Lemma 3.11 Let $w \in X$ be satisfying $\ell(\boldsymbol{w})=0$ a.e. in the open set $\mathcal{O} \subset \Omega$; then there exists a family $\boldsymbol{w}^{\varepsilon} \in X, \varepsilon \in E$, such that

$$
\ell\left(\boldsymbol{w}^{\varepsilon}\right)=0 \text { in } \mathcal{O}, \quad \lim _{\varepsilon \downarrow 0}\left(\varepsilon \int_{\mathcal{O}} \boldsymbol{q}\left(x, \nabla \boldsymbol{w}^{\varepsilon}(x)\right) \mathrm{d} x+\int_{\Omega}\left|\boldsymbol{w}^{\varepsilon}-\boldsymbol{w}\right|^{2} \mathrm{~d} x\right)=0 .
$$


Proof. Of course, we simply have to define $\boldsymbol{w}^{\varepsilon}$ in $\mathcal{O}$, since we can set $\boldsymbol{w}^{\varepsilon} \equiv \boldsymbol{w}$ in $\Omega \backslash \mathcal{O}$. Then we can invoke the density of $H^{1}\left(\mathcal{O} ; \mathbb{R}^{m}\right)$ in $L^{2}\left(\mathcal{O} ; \mathbb{R}^{m}\right)$ to find $\tilde{\boldsymbol{w}}^{\varepsilon} \in H^{1}\left(\mathcal{O} ; \mathbb{R}^{m}\right)$ satisfying (3.31). Taking $\boldsymbol{w}^{\varepsilon}:=P\left(\tilde{\boldsymbol{w}}^{\varepsilon}\right), P: \mathbb{R}^{m} \rightarrow \mathbb{R}^{m}$ being the orthogonal projection on the kernel of $\ell$, we conclude.

Proposition 3.12 Let $\mathcal{O} \in \boldsymbol{A}(\Omega)$ and $\boldsymbol{u}, \boldsymbol{v}$ be two functions in $X$ satisfying

$$
u=\ell(\boldsymbol{u})=\ell(\boldsymbol{v})=v \text { a.e. in } \mathcal{O} .
$$

Then we have

$$
F^{\prime}(\boldsymbol{u}, \mathcal{O})=F^{\prime}(\boldsymbol{v}, \mathcal{O}), \quad F^{\prime \prime}(\boldsymbol{u}, \mathcal{O})=F^{\prime \prime}(\boldsymbol{v}, \mathcal{O})
$$

Proof. Let $\left\{\boldsymbol{u}^{\varepsilon}\right\}_{\varepsilon \in E}$ be a family such that

$$
\lim _{\varepsilon \downarrow 0} \boldsymbol{u}^{\varepsilon}=\boldsymbol{u} \quad \text { in } X .
$$

By applying the previous lemma to the function $\boldsymbol{w}:=\boldsymbol{v}-\boldsymbol{u}$ we find $\boldsymbol{w}^{\varepsilon} \in X \cap H^{1}\left(\mathcal{O} ; \mathbb{R}^{m}\right), \varepsilon \in E$, such that $\ell\left(\boldsymbol{w}^{\varepsilon}\right)=0$ in $\mathcal{O}$ and (3.31) holds.

Setting $\boldsymbol{v}^{\varepsilon}:=\boldsymbol{u}^{\varepsilon}+\boldsymbol{w}^{\varepsilon}$, we obtain $\ell\left(\boldsymbol{v}^{\varepsilon}\right)=\ell\left(\boldsymbol{u}^{\varepsilon}\right), \lim _{\varepsilon \in E} \boldsymbol{v}^{\varepsilon}=\boldsymbol{v}$ in $X$. In order to bound $\boldsymbol{q}\left(x, \nabla \boldsymbol{w}^{\varepsilon}\right)$, let us recall that Cauchy-Schwarz inequality yields for every $x \in \Omega$

$$
\boldsymbol{q}(x, \mathfrak{a}+\mathfrak{b}) \leqslant(1+\delta) \boldsymbol{q}(x, \mathfrak{a})+\left(1+\delta^{-1}\right) \boldsymbol{q}(x, \mathfrak{b}), \quad \forall \mathfrak{a}, \mathfrak{b} \in \mathbb{M}^{m \times n}, \forall \delta>0 .
$$

In particular, we have (the inequality is trivial if $\left.\boldsymbol{u}^{\varepsilon}\right|_{\mathcal{O}} \notin H^{1}\left(\mathcal{O} ; \mathbb{R}^{m}\right)$ )

$$
F^{\varepsilon}\left(\boldsymbol{v}^{\varepsilon} ; \mathcal{O}\right) \leqslant(1+\delta) F^{\varepsilon}\left(\boldsymbol{u}^{\varepsilon} ; \mathcal{O}\right)+\left(1+\delta^{-1}\right) \varepsilon \int_{\mathcal{O}} \boldsymbol{q}\left(x, \nabla \boldsymbol{w}^{\varepsilon}\right) \mathrm{d} x,
$$

which implies, by passing to the limit as $\varepsilon \downarrow 0$ and taking $\delta$ arbitrarily small,

$$
\liminf _{\varepsilon \downarrow 0} F^{\varepsilon}\left(\boldsymbol{v}^{\varepsilon}\right) \leqslant \liminf _{\varepsilon \downarrow 0} F^{\varepsilon}\left(\boldsymbol{u}^{\varepsilon}\right), \quad \limsup _{\varepsilon \downarrow 0} F^{\varepsilon}\left(\boldsymbol{v}^{\varepsilon}\right) \leqslant \limsup _{\varepsilon \downarrow 0} F^{\varepsilon}\left(\boldsymbol{u}^{\varepsilon}\right) .
$$

Recalling definitions (2.5a) and (2.5b), we get

$$
F^{\prime}(\boldsymbol{v} ; \mathcal{O}) \leqslant F^{\prime}(\boldsymbol{u} ; \mathcal{O}), \quad F^{\prime \prime}(\boldsymbol{v} ; \mathcal{O}) \leqslant F^{\prime \prime}(\boldsymbol{u} ; \mathcal{O}) .
$$

Changing the role of $\boldsymbol{u}$ and $\boldsymbol{v}$, we conclude.

By this result, the following definition is well posed.

Definition 3.13 For every $u \in L^{2}(\Omega), \mathcal{O} \in \boldsymbol{A}(\Omega)$, we set

$$
G^{\prime}(u, \mathcal{O}):=F^{\prime}(\boldsymbol{u}, \mathcal{O}), \quad G^{\prime \prime}(u, \mathcal{O}):=F^{\prime \prime}(\boldsymbol{u}, \mathcal{O})
$$

whenever $u=\ell(\boldsymbol{u})$ a.e. in $\mathcal{O}$. Moreover, if $F^{\varepsilon} \bar{\Gamma}(X)$-converges to $\bar{F}$ as $\varepsilon \downarrow 0$, then we set

$$
\bar{G}(u, \mathcal{O}):=\bar{F}(\boldsymbol{u}, \mathcal{O}), \quad \forall u \in L^{2}(\Omega), \mathcal{O} \in A(\Omega) .
$$


We summarize some of the properties of $G^{\prime}, G^{\prime \prime}$ which have just been proved, in the following lemma (cf. paragraph 2.2).

Lemma 3.14 The functionals $G^{\prime}, G^{\prime \prime}: L^{2}(\Omega) \times \boldsymbol{A}(\Omega) \rightarrow[0,+\infty]$ are local, increasing, and lower semicontinuous; $G^{\prime}$ is superadditive, $G^{\prime \prime}$ is weakly subadditive, and $\bar{G}$ (when it exists) is a measure. Moreover, they satisfy for every Lipschitz open set $\mathcal{O} \in \boldsymbol{A}(\Omega)$

$$
G^{\prime}(u, \mathcal{O})<+\infty \quad \Leftrightarrow \quad G^{\prime \prime}(u, \mathcal{O})<+\infty \quad \Leftrightarrow \quad u_{\left.\right|_{\mathcal{O}}} \in B V(\mathcal{O} ; T)
$$

and, for the same constants $\lambda_{0}, \Lambda_{0}$ defined above,

$$
\lambda_{0} H^{n-1}\left(S_{u}^{*} \cap \mathcal{O}\right) \leqslant G^{\prime}(u, \mathcal{O}) \leqslant G^{\prime \prime}(u, \mathcal{O}) \leqslant \Lambda_{0} H^{n-1}\left(S_{u}^{*} \cap \mathcal{O}\right) .
$$

Finally, (3.36) and (3.37) hold also for $\bar{G}$ and every open set.

\subsection{Lipschitz subsets}

Let us start with a simple modification of Corollary 3.9.

COROLlary 3.15 Let $\boldsymbol{u} \in X$ and $\mathcal{O}_{1}, \mathcal{O}_{1}^{\prime}, \mathcal{O}_{2} \in \boldsymbol{A}(\Omega)$ with $\mathcal{O}_{1} \Subset \mathcal{O}_{1}^{\prime}$. Then

$$
F^{\prime}\left(\boldsymbol{u}, \mathcal{O}_{1} \cup \mathcal{O}_{2}\right) \leqslant F^{\prime}\left(\boldsymbol{u}, \mathcal{O}_{1}^{\prime}\right)+F^{\prime \prime}\left(\boldsymbol{u}, \mathcal{O}_{2}\right)
$$

and

$$
F^{\prime}\left(\boldsymbol{u}, \mathcal{O}_{1} \cup \mathcal{O}_{2}\right) \leqslant F^{\prime \prime}\left(\boldsymbol{u}, \mathcal{O}_{1}^{\prime}\right)+F^{\prime}\left(\boldsymbol{u}, \mathcal{O}_{2}\right) .
$$

Proof. Equation (3.38a) follows as for the previous corollary, by substituting (3.29) with

$$
\begin{aligned}
\liminf _{\varepsilon \downarrow 0, \varepsilon \in E} F^{\varepsilon}\left(\boldsymbol{w}^{\varepsilon}, \mathcal{O}_{1} \cup \mathcal{O}_{2}\right) & \leqslant \liminf _{\varepsilon \downarrow 0, \varepsilon \in E}\left(F^{\varepsilon}\left(\boldsymbol{u}^{\varepsilon}, \mathcal{O}_{1}^{\prime}\right)+F^{\varepsilon}\left(\boldsymbol{v}^{\varepsilon}, \mathcal{O}_{2}\right)\right) \\
& \leqslant \liminf _{\varepsilon \downarrow 0, \varepsilon \in E} F^{\varepsilon}\left(\boldsymbol{u}^{\varepsilon}, \mathcal{O}_{1}^{\prime}\right)+\limsup _{\varepsilon \downarrow 0, \varepsilon \in E} F^{\varepsilon}\left(\boldsymbol{v}^{\varepsilon}, \mathcal{O}_{2}\right) .
\end{aligned}
$$

Analogously, (3.38b) follows by the other estimate

$$
\begin{aligned}
\liminf _{\varepsilon \downarrow 0, \varepsilon \in E} F^{\varepsilon}\left(\boldsymbol{w}^{\varepsilon}, \mathcal{O}_{1} \cup \mathcal{O}_{2}\right) & \leqslant \liminf _{\varepsilon \downarrow 0, \varepsilon \in E}\left(F^{\varepsilon}\left(\boldsymbol{u}^{\varepsilon}, \mathcal{O}_{1}^{\prime}\right)+F^{\varepsilon}\left(\boldsymbol{v}^{\varepsilon}, \mathcal{O}_{2}\right)\right) \\
& \leqslant \limsup _{\varepsilon \downarrow 0, \varepsilon \in E} F^{\varepsilon}\left(\boldsymbol{u}^{\varepsilon}, \mathcal{O}_{1}^{\prime}\right)+\liminf _{\varepsilon \downarrow 0, \varepsilon \in E} F^{\varepsilon}\left(\boldsymbol{v}^{\varepsilon}, \mathcal{O}_{2}\right) .
\end{aligned}
$$

COROLlaRY 3.16 If $\mathcal{O} \in \boldsymbol{A}(\Omega)$ is Lipschitz then $F^{\prime}(\cdot, \mathcal{O})$ and $F^{\prime \prime}(\cdot, \mathcal{O})$ coincide with their inner regular envelopes (cf. Section 2.3); in particular, if there exists the $\bar{\Gamma}(X)$-limit of $F^{\varepsilon}$, then $F^{\prime}(\cdot, \mathcal{O})=F^{\prime \prime}(\cdot, \mathcal{O})$.

Proof. Let us first consider the case of the superior $\Gamma$-limit $F^{\prime \prime}$ : we fix $\mathcal{O} \in \boldsymbol{A}(\Omega)$ and we choose $\mathcal{O}_{1} \Subset \mathcal{O}_{2} \Subset \mathcal{O}, \mathcal{O}_{1}$ Lipschitz. By the weak subadditivity and Lemma 3.4, we get, for every $\boldsymbol{u} \in X$, with $u_{\left.\right|_{\mathcal{O}}} \in B V(\mathcal{O} ; T)$ for $u:=\ell(\boldsymbol{u})$

$$
\begin{aligned}
F^{\prime \prime}(\boldsymbol{u}, \mathcal{O}) & \leqslant F^{\prime \prime}\left(\boldsymbol{u}, \mathcal{O}_{2}\right)+F^{\prime \prime}\left(\boldsymbol{u}, \mathcal{O} \backslash \overline{\mathcal{O}}_{1}\right) \\
& \leqslant F^{\prime \prime}\left(\boldsymbol{u}, \mathcal{O}_{2}\right)+\Lambda_{0} H^{n-1}\left(S_{u}^{*} \cap\left(\mathcal{O} \backslash \mathcal{O}_{1}\right)\right) .
\end{aligned}
$$


Therefore,

$$
F^{\prime \prime}(\boldsymbol{u}, \mathcal{O}) \leqslant \sup _{\mathcal{O}_{2} \Subset \mathcal{O}} F^{\prime \prime}\left(\boldsymbol{u}, \mathcal{O}_{2}\right)+c^{\prime \prime} H^{n-1}\left(S_{u}^{*} \cap\left(\mathcal{O} \backslash \mathcal{O}_{1}\right)\right)
$$

As $\mathcal{O}_{1} \Subset \mathcal{O}$ an arbitrary Lipschitz open set, we conclude. The proof of the corresponding result for $F^{\prime}$ follows by the same argument and applying (3.38a).

\subsection{Representation results}

In this section we apply the additivity result of Corollary 3.10 and the representation results of [2] quoted in Section 2.6 to obtain formula (1.24) of Theorem 1.1, assuming the existence of the $\bar{\Gamma}(X)$ limit $\bar{F}$. As before, we set $X:=L^{2}\left(\Omega ; \mathbb{R}^{m}\right)$.

First of all we prove a continuous dependence result under small perturbations of the open set which is related to $(2.18)$. Let us first recall that, by the continuity assumptions on $\boldsymbol{q}$, there exists a continuous function $\omega_{\boldsymbol{q}}:\left[0,+\infty\left[\rightarrow\left[0,+\infty\left[\right.\right.\right.\right.$ such that $\omega_{\boldsymbol{q}}(0)=0$ and

$$
|\boldsymbol{q}(x, \mathfrak{a})-\boldsymbol{q}(y, \mathfrak{a})| \leqslant \omega_{\boldsymbol{q}}(|x-y|) \boldsymbol{q}(x, \mathfrak{a}), \quad \forall x, y \in \Omega, \quad \mathfrak{a} \in \mathbb{M}^{m \times n} .
$$

LEMMA 3.17 For every $\mathcal{O} \Subset \Omega$ there exists a continuous function $\omega:[0,+\infty[\rightarrow[0,+\infty[$ such that $\omega(0)=0$ and

$$
\left|G^{\prime}\left(u, \mathcal{O}_{1}\right)-G^{\prime}\left(v, \mathcal{O}_{1}+z\right)\right| \leqslant \omega(|z|) H^{n-1}\left(\mathcal{O}_{1} \cap S_{u}^{*}\right)
$$

whenever $\mathcal{O}_{1} \subset \mathcal{O}, z \in \mathbb{R}^{n},|z|<\frac{1}{2} d(\mathcal{O}, \partial \Omega)$ and $v(x+z)=u(x)$ in $\mathcal{O}_{1}$. The same property holds also for $G^{\prime \prime}$.

Proof. Let us observe that for every $\boldsymbol{w} \in H^{1}\left(\mathcal{O} ; \mathbb{R}^{m}\right) \cap L^{p_{0}}\left(\mathcal{O} ; \mathbb{R}^{m}\right), \boldsymbol{w}_{z}(x):=\boldsymbol{w}(x-z)$, we have

$$
\begin{aligned}
\left|F^{\varepsilon}(\boldsymbol{w}, \mathcal{O})-F^{\varepsilon}\left(\boldsymbol{w}_{z}, \mathcal{O}+z\right)\right| & =\varepsilon\left|\int_{\mathcal{O}}(\boldsymbol{q}(x, \nabla \boldsymbol{w}(x))-\boldsymbol{q}(x+z, \nabla \boldsymbol{w}(x))) \mathrm{d} x\right| \\
& \leqslant \omega(|z|) \int_{\mathcal{O}} \varepsilon \boldsymbol{q}(x, \nabla \boldsymbol{w}(x)) \mathrm{d} x \leqslant \omega(|z|) F^{\varepsilon}(\boldsymbol{w}, \mathcal{O}) .
\end{aligned}
$$

Now we choose $u \in B V(\Omega ; T), \delta>0$, and $\boldsymbol{u}^{\varepsilon} \in X, \varepsilon \in E$, such that

$$
\boldsymbol{u}^{\varepsilon} \rightarrow \boldsymbol{u}, \quad \text { in } X, \quad \ell(\boldsymbol{u})=u, \quad \liminf _{\varepsilon \in D} F^{\varepsilon}\left(\boldsymbol{u}^{\varepsilon}, \mathcal{O}\right) \leqslant G^{\prime}(u, \mathcal{O})+\delta .
$$

Choosing $z \in \mathbb{R}^{n}$ with $|z|<\frac{1}{2} d(\mathcal{O}, \partial \Omega), \boldsymbol{v}^{\varepsilon}(x)=\boldsymbol{u}^{\varepsilon}(x-z)$, we deduce

$$
\begin{aligned}
G^{\prime}(v, \mathcal{O}+z)-G^{\prime}(u, \mathcal{O}) & \leqslant \omega(|z|) \liminf _{\varepsilon \in D} F^{\varepsilon}\left(\boldsymbol{u}^{\varepsilon}, \mathcal{O}\right)+\delta \\
& \leqslant \omega(|z|)\left(G^{\prime}(u, \mathcal{O})+\delta\right)+\delta \\
& \leqslant \delta+\omega(|z|)\left(\delta+\Lambda_{0} H^{n-1}\left(S_{u}^{*} \cap \mathcal{O}\right)\right) .
\end{aligned}
$$

As $\delta>0$ is arbitrary, and changing the role of $u$ and $v$, we complete the proof for the lower $\Gamma$-limits; an analogous argument covers the case of $G^{\prime \prime}$. 
Corollary 3.18 Let assume that there exists the $\bar{\Gamma}(X)$-limit $\bar{F}$ of $F^{\varepsilon}$ as $\varepsilon \downarrow 0$ in $E$, and let us set, as usual, $\bar{G}(u, \cdot)=\bar{F}(\boldsymbol{u}, \cdot)$ whenever $u=\ell(\boldsymbol{u})$. Then there exists a continuous family of norms $\phi(x, \cdot), x \in \Omega$, such that for every $u \in B V(\Omega ; T)$ and $\mathcal{O} \in \boldsymbol{A}(\Omega)$

$$
\bar{G}(u, \mathcal{O})=\int_{S_{u}^{*} \cap \mathcal{O}} \phi\left(x, v_{u}(x)\right) \mathrm{d} H^{n-1}(x) .
$$

Proof. We have simply to apply the result quoted in Section 2.6 to $\bar{G}: L^{2}(\Omega) \times \boldsymbol{A}(\Omega)$, which is local, lower semicontinuous, a measure in the sense of Section 2.2, and satisfies (2.17) by Lemma 3.14; in order to check also (2.18), we simply observe that

$$
\bar{G}(u, \mathcal{O})=\sup _{\mathcal{O}_{1} \Subset \mathcal{O}} G^{\prime}\left(u, \mathcal{O}_{1}\right)
$$

and we apply Corollary 3.17 .

\subsection{Characterization of $\phi$}

The last step in the proof of Theorem 1.1 is to show that $\phi$ is independent of $E$ : if this is the case, we will obtain the $\bar{\Gamma}$-convergence of the whole family $F^{\varepsilon}, \varepsilon>0$. We will prove this uniqueness by showing 1.25 .

Admissible boundary conditions First of all, we address the questions posed by the following definition.

Definition 3.19 Let us fix $\mathcal{O} \in \boldsymbol{A}(\Omega)$ and $u \in L^{2}(\Omega) \cap B V(\mathcal{O} ; T)$, and let us consider a subset $H$ of $H^{1}\left(\mathcal{O} ; \mathbb{R}^{m}\right)$ such that

$$
H+H_{0}^{1}\left(\mathcal{O} ; \mathbb{R}^{m}\right) \subset H .
$$

We say that $H$ is $X$-admissible for $u$ if for every family $\boldsymbol{u}^{\varepsilon}, \varepsilon \in E$, converging to $\boldsymbol{u}$ in $X, \ell(\boldsymbol{u})=u$ in $\mathcal{O}$, it is possible to find $\boldsymbol{w}^{\varepsilon} \in X$ converging to $\boldsymbol{w}$ such that $\ell(\boldsymbol{w})=u$ in $\mathcal{O}$ and

$$
\left.\boldsymbol{w}^{\varepsilon}\right|_{\mathcal{O}} \in H, \quad \limsup _{\varepsilon \downarrow 0, \varepsilon \in E} F^{\varepsilon}\left(\boldsymbol{w}^{\varepsilon}, \mathcal{O}\right) \leqslant \limsup _{\varepsilon \downarrow 0, \varepsilon \in E} F^{\varepsilon}\left(\boldsymbol{u}^{\varepsilon}, \mathcal{O}\right) .
$$

REMARK 3.20 The previous property can also be reformulated in terms of the indicator function $\mathcal{I}_{H}$ of $H$, which is defined by

$$
\mathcal{I}_{H}(\boldsymbol{u})= \begin{cases}0 & \text { if } \boldsymbol{u} \in H, \\ +\infty & \text { otherwise }\end{cases}
$$

Then $H$ is admissible for $u$ if the $\Gamma(X)$-limits as $\varepsilon \downarrow 0$ in $E$ of $F^{\varepsilon}(\cdot, \mathcal{O})$ and $F^{\varepsilon}(\cdot, \mathcal{O})+\mathcal{I}_{H}$ coincide at every $\boldsymbol{u} \in X$ with $\ell(\boldsymbol{u})=u$ in $\mathcal{O}$.

The next result, which is an abstract formulation of some ideas hidden in [4: Lemma 3.2], provides a useful tool when we want to 'change the boundary conditions' of a local minimizing sequence. 
Proposition 3.21 Let $\mathcal{O}$ be an open subset of $\Omega$, let $\boldsymbol{u} \in X$ be a function such that the restriction of $u=\ell(\boldsymbol{u})$ to $\mathcal{O}$ belongs to $B V(\mathcal{O} ; T)$, and let $H \subset H^{1}\left(\mathcal{O} ; \mathbb{R}^{m}\right)$ satisfy (3.44). If there exists a family $\boldsymbol{v}^{\varepsilon} \in H, \varepsilon \in E$, converging to $\boldsymbol{v}$ in $L^{2}\left(\Omega ; \mathbb{R}^{m}\right)$ such that

$$
\ell(\boldsymbol{v})=u \text { in } \mathcal{O}, \quad \inf _{\mathcal{Q} \Subset \mathcal{O}}\left\{\limsup _{\varepsilon \downarrow 0} F^{\varepsilon}\left(\boldsymbol{v}^{\varepsilon}, \mathcal{O} \backslash \mathcal{Q}\right)\right\}=0,
$$

then $H$ is admissible for $\boldsymbol{u}$.

Proof. Let us fix $\delta>0$, an open set $\mathcal{Q}_{\delta}, \mathcal{Q}_{\delta}^{\prime}$ such that $\mathcal{Q}_{\delta} \Subset \mathcal{Q}_{\delta}^{\prime} \Subset \mathcal{O}$, and a family $\boldsymbol{v}^{\varepsilon}$ as in the statement of the proposition satisfying

$$
\underset{\varepsilon \downarrow 0}{\limsup } F^{\varepsilon}\left(\boldsymbol{v}^{\varepsilon}, \mathcal{O} \backslash \mathcal{Q}\right) \leqslant \delta, \quad \sup _{x \in \mathcal{Q}_{\delta}} d(x, \partial \mathcal{O}) \leqslant \delta .
$$

We consider a family $\boldsymbol{u}^{\varepsilon}, \varepsilon \in E$, as in Definition 3.19 (we first consider the case of the strong convergence) and we apply Lemma 3.6 with

$$
\mathcal{O}_{1}:=\mathcal{Q}_{\delta}, \quad \mathcal{O}_{1}^{\prime}:=\mathcal{Q}_{\delta}^{\prime}, \quad \mathcal{O}_{2}:=\mathcal{O} \backslash \mathcal{Q}_{\delta} .
$$

We find a family $\boldsymbol{w}^{\varepsilon, \delta}$ such that

$$
\lim _{\varepsilon \downarrow 0, \varepsilon \in E} \boldsymbol{w}^{\varepsilon, \delta}=\boldsymbol{u} \text { in } X, \text { uniformly w.r.t. } \delta,
$$

$\boldsymbol{w}^{\varepsilon, \delta}-\boldsymbol{v}^{\varepsilon} \in H_{0}^{1}\left(\mathcal{O} ; \mathbb{R}^{m}\right)$ so that $\left.\boldsymbol{w}^{\varepsilon, \delta}\right|_{\mathcal{O}} \in H$, and

$$
\begin{aligned}
\limsup _{\varepsilon \downarrow 0, \varepsilon \in E} F^{\varepsilon}\left(\boldsymbol{w}^{\varepsilon, \delta}, \mathcal{O}\right) & \leqslant \limsup _{\varepsilon \downarrow 0, \varepsilon \in E} F^{\varepsilon}\left(\boldsymbol{u}^{\varepsilon}, \mathcal{Q}_{\delta}^{\prime}\right)+\limsup _{\varepsilon \downarrow 0, \varepsilon \in E} F^{\varepsilon}\left(\boldsymbol{v}^{\varepsilon}, \mathcal{O} \backslash \mathcal{Q}_{\delta}\right) \\
& \leqslant \limsup _{\varepsilon \downarrow 0, \varepsilon \in E} F^{\varepsilon}\left(\boldsymbol{u}^{\varepsilon}, \mathcal{O}\right)+\delta .
\end{aligned}
$$

Defining now $\boldsymbol{w}^{\varepsilon}:=\boldsymbol{w}^{\varepsilon, \varepsilon}$ we conclude the proof.

Characterization of $\phi$ when $\boldsymbol{q}$ is independent of $x$ As we shall see at the end of this section, it is not difficult to reduce the problem to the case of a single quadratic form $\boldsymbol{q}$, independent of $x$; therefore, for the time being we assume that

$$
\boldsymbol{q}(x, \mathfrak{a}) \equiv \boldsymbol{q}(\mathfrak{a}), \quad \text { constant w.r.t. } x \in \Omega .
$$

Lemma 3.22 Let $\phi$ be the function defined in Corollary 3.18, $v \in \mathbb{S}^{n-1}$, and $Q$ be an admissible set for $v$ as in (1.20). Then

$$
\phi(v) \geqslant \inf \left\{\int_{Q} f^{\varepsilon}(\boldsymbol{u}, \nabla \boldsymbol{u}): \boldsymbol{u} \in \mathcal{B}\left(Q ; \mathbb{R}^{m}\right), \varepsilon>0\right\} .
$$

Proof. It is not restrictive to assume $Q \subset \Omega$; we introduce the functions

$$
j_{\varepsilon}(t):= \begin{cases}-1 & \text { if } t<-\varepsilon \\ t / \varepsilon & \text { if }-\varepsilon \leqslant t \leqslant \varepsilon \quad j_{0}(t):=\operatorname{sign}(t), \\ 1 & \text { if } t>\varepsilon\end{cases}
$$


and we define

$$
u(x):=\frac{1}{2}\left(s_{-}+s_{+}\right)+\frac{1}{2}\left(s_{+}-s_{-}\right) j(\langle x, v\rangle) .
$$

By (3.43) we easily have

$$
\bar{G}(u, Q)=G^{\prime}(u, Q)=G^{\prime \prime}(u, Q)=\phi(v) .
$$

The thesis follows if we show that $\mathcal{B}\left(Q ; \mathbb{R}^{m}\right)$ is admissible for $u$, as Definition 3.19 and Corollary 3.16 yield

$$
\inf \left\{\limsup _{\varepsilon \downarrow 0} F^{\varepsilon}\left(\boldsymbol{u}^{\varepsilon}, Q\right): \boldsymbol{u}^{\varepsilon} \rightarrow \boldsymbol{u} \text { in } X, \boldsymbol{u}^{\varepsilon} \in \mathcal{B}\left(Q ; \mathbb{R}^{m}\right)\right\}=G^{\prime \prime}(u, Q)=\phi(\nu) .
$$

This relation obviously implies (3.47).

In order to prove the admissibility of $\mathcal{B}\left(Q ; \mathbb{R}^{m}\right)$, we apply Proposition 3.21: we choose two points $\boldsymbol{s}_{ \pm} \in \mathbb{R}^{m}$ such that $\ell\left(\boldsymbol{s}_{ \pm}\right)=s_{ \pm}$and we set

$$
\boldsymbol{v}^{\varepsilon}(x):=\frac{1}{2}\left(\boldsymbol{s}_{-}+\boldsymbol{s}_{+}\right)+\frac{1}{2}\left(\boldsymbol{s}_{+}-\boldsymbol{s}_{-}\right) j_{\varepsilon}(\langle x, v\rangle)
$$

which obviously belong to $\mathcal{B}\left(Q ; \mathbb{R}^{m}\right)$. Choosing, e.g., $Q_{\rho}:=\rho Q, \rho<1$, a simple calculation shows that

$$
F^{\varepsilon}\left(\boldsymbol{v}^{\varepsilon}, Q \backslash Q_{\rho}\right) \leqslant H^{n-1}\left(Q \backslash Q_{\rho}\right)\left(\Lambda\left|s_{+}-s_{-}\right|^{2}+\max \left(W\left(s_{-}\right), W\left(s_{+}\right)\right)\right) .
$$

Letting $\rho \uparrow 1$, we get (3.45).

Now we prove the opposite inequality: this concludes the proof of Theorem 1.1 in the constant coefficient case.

Lemma 3.23 Let $\phi, v, Q$ be as in the previous lemma, let $\boldsymbol{u} \in L^{2}\left(\Omega ; \mathbb{R}^{m}\right)$ be a function of $\mathcal{B}\left(Q ; \mathbb{R}^{m}\right)$, and $\varepsilon_{0}>0$. Then

$$
\phi(v) \leqslant \int_{Q} f^{\varepsilon_{0}}(\boldsymbol{u}(x), \nabla \boldsymbol{u}(x)) \mathrm{d} x .
$$

Proof. Let $\boldsymbol{s}_{-}, \boldsymbol{s}_{+}$be the (constant) traces of $\boldsymbol{u}$ on the basis $Q^{ \pm}$, which by definition of $\mathcal{B}\left(Q ; \mathbb{R}^{n}\right)$ satisfy $\ell\left(\boldsymbol{s}_{ \pm}\right)=s_{ \pm}$, and for every $r \in \mathbb{R}$ let us denote by $r^{*}$ the unique element of $[-1 / 2,1 / 2$ [ such that $r-r^{*}$ is an integer.

Denoting a point $x \in \mathbb{R}^{n}$ with its coordinates $r_{1}, \ldots, r_{n}$ with respect to the basis $\eta_{1}, \ldots, \eta_{n-1}, \eta$ of $\mathbb{R}^{n}$ introduced in (1.20), we extend $\boldsymbol{u}$ to the whole $\mathbb{R}^{n}$ by setting

$$
\boldsymbol{u}\left(r_{1}, \ldots, r_{n}\right):= \begin{cases}\boldsymbol{s}_{-} & \text {if } r_{n} \leqslant a, \\ \boldsymbol{u}\left(r_{1}^{*}, r_{2}^{*}, \ldots, r_{n-1}^{*}, r_{n}\right) & \text { if } a<r_{n}<b, \\ s s_{+} & \text {if } r_{n}>b .\end{cases}
$$

Therefore, $\boldsymbol{u}$ is the unique $H_{l o c}^{1}\left(\mathbb{R}^{n} ; \mathbb{R}^{m}\right)$ extension which is periodic along the directions $\eta_{1}, \ldots, \eta_{n-1}$ and constant outside the strip $a<r_{n}<b$. 
We fix an odd integer $k=2 h+1, h \in \mathbb{N}$, and we define

$$
\boldsymbol{u}^{k}:=\boldsymbol{u}(k x) \quad \text { which belongs to } \mathcal{B}\left(Q ; \mathbb{R}^{m}\right) \text {, too. }
$$

It is easy to see that

$$
\begin{aligned}
\int_{Q} \boldsymbol{q}\left(\nabla \boldsymbol{u}^{k}(x)\right) \mathrm{d} x & =\int_{Q} k^{2} \boldsymbol{q}(\nabla \boldsymbol{u}(k x)) \mathrm{d} x \\
& =\int_{k Q} k^{2} \boldsymbol{q}(\nabla \boldsymbol{u}(y)) k^{-n} \mathrm{~d} y \\
& =k^{n-1} \int_{Q} k^{2-n} \boldsymbol{q}(\nabla \boldsymbol{u}(y)) \mathrm{d} y \\
& =k \int_{Q} \boldsymbol{q}(\nabla \boldsymbol{u}(x)) \mathrm{d} x
\end{aligned}
$$

and

$$
\begin{aligned}
\int_{Q} W\left(u^{k}(x)\right) \mathrm{d} x & =\int_{Q} W(u(k x)) \mathrm{d} x \\
& =\int_{k Q} W(u(y)) k^{-n} \mathrm{~d} y \\
& =k^{n-1} \int_{Q} W(u(y)) k^{-n} \mathrm{~d} y \\
& =k^{-1} \int_{Q} W(u(y)) \mathrm{d} y .
\end{aligned}
$$

Therefore,

$$
F^{\varepsilon}\left(\boldsymbol{u}^{k}, Q\right)=F^{\varepsilon k}(\boldsymbol{u}, Q) .
$$

Choosing now $k:=k(\varepsilon)$ as the (odd) integer part of $\varepsilon_{0} / \varepsilon, \varepsilon \in E$, it is obvious that

$$
\begin{gathered}
F^{\varepsilon}\left(\boldsymbol{u}^{k(\varepsilon)}, Q\right)=F^{\varepsilon k(\varepsilon)}(\boldsymbol{u}, Q) \rightarrow F^{\varepsilon_{0}}(\boldsymbol{u}, Q), \\
\boldsymbol{u}^{k(\varepsilon)} \rightarrow \boldsymbol{u}:=\frac{1}{2}\left(\boldsymbol{s}_{-}+\boldsymbol{s}_{+}\right)+\frac{1}{2}\left(\boldsymbol{s}_{+}-\boldsymbol{s}_{-}\right) j(\langle x, \nu\rangle)
\end{gathered}
$$

in $L^{2}\left(Q ; \mathbb{R}^{m}\right)$ as $\varepsilon \downarrow 0, j$ being defined in (3.48). On the other hand, we have as in (3.50),

$$
\phi(v)=F^{\prime}(\boldsymbol{u}, Q) \leqslant \liminf _{\varepsilon \downarrow 0, \varepsilon \in E} F^{\varepsilon}\left(\boldsymbol{u}^{k_{\varepsilon}}, Q\right) .
$$

Corollary 3.24 Let us suppose that $\boldsymbol{q}(\cdot, \mathfrak{a}) \equiv \boldsymbol{q}(\mathfrak{a})$ is constant; then the family $F^{\varepsilon}(\boldsymbol{u}, \cdot), \varepsilon>0$, $\bar{\Gamma}(X)$ converges to the functional $G(\ell(\boldsymbol{u}), \cdot)$, where

$$
G(u, \mathcal{O}):=\int_{S_{u}^{*} \cap \mathcal{O}} \phi\left(v_{u}(x)\right) \mathrm{d} H^{n-1}(x),
$$

where $\phi$ is defined as in Theorem 1.1. Moreover, if $\mathcal{O}$ is Lipschitz, we have

$$
F^{\prime}(\boldsymbol{u}, \mathcal{O})=F^{\prime \prime}(\boldsymbol{u}, \mathcal{O})=G(\ell(\boldsymbol{u}), \mathcal{O})
$$


Characterization of $\phi$ in the general case We conclude now the proof of an analogous result in the non-constant coefficient case, by adapting the same arguments of Lemma 3.17.

Lemma 3.25 For every $x_{0} \in \Omega$, let us denote by $F_{x_{0}}^{\varepsilon}$

$$
F_{x_{0}}^{\varepsilon}(\boldsymbol{u}, \mathcal{O}):=\int_{\mathcal{O}} f^{\varepsilon}\left(x_{0}, \boldsymbol{u}(x), \nabla \boldsymbol{u}(x)\right) \mathrm{d} x,
$$

and by $F_{x_{0}}$ its $\bar{\Gamma}$-limit. For every Lipschitz open set $\mathcal{O}$ and $\boldsymbol{w} \in L^{2}\left(\Omega ; \mathbb{R}^{m}\right)$ we have

$$
\left|F^{\prime}(\boldsymbol{w}, \mathcal{O})-F_{x_{0}}(\boldsymbol{w}, \mathcal{O})\right| \leqslant \vartheta(\mathcal{O}) H^{n-1}\left(S_{w}^{*} \cap \mathcal{O}\right),
$$

where $\vartheta(\mathcal{O}):=\Lambda_{0} \sup _{x \in \mathcal{O}} \omega\left(\left|x-x_{0}\right|\right)$. The same bound holds also for the superior $\Gamma$-limit $F^{\prime \prime}$.

Proof. As in Lemma 3.17 we get for every $\boldsymbol{w} \in H^{1}\left(\mathcal{O} ; \mathbb{R}^{m}\right) \cap L^{p_{0}}\left(\mathcal{O} ; \mathbb{R}^{m}\right)$

$$
\begin{aligned}
\left|F^{\varepsilon}(\boldsymbol{w}, \mathcal{O})-F_{x_{0}}^{\varepsilon}(\boldsymbol{w}, \mathcal{O})\right| & =\varepsilon\left|\int_{\mathcal{O}}\left(\boldsymbol{q}(x, \nabla \boldsymbol{w}(x))-\boldsymbol{q}\left(x_{0}, \nabla \boldsymbol{w}(x)\right)\right) \mathrm{d} x\right| \\
& \leqslant \vartheta(\mathcal{O}) \int_{\mathcal{O}} \varepsilon \boldsymbol{q}\left(x_{0}, \nabla \boldsymbol{w}(x)\right) \mathrm{d} x \leqslant \vartheta(\mathcal{O}) F_{x_{0}}^{\varepsilon}(\boldsymbol{w}, \mathcal{O}) .
\end{aligned}
$$

The proof then follows as in Lemma 3.17

COROLlary 3.26 The family $F^{\varepsilon}(\boldsymbol{u}, \cdot) \bar{\Gamma}(X)$ converges to the functional $G(\ell(\boldsymbol{u}), \cdot)$, where

$$
G(u, \mathcal{O}):=\int_{S_{u}^{*} \cap \mathcal{O}} \phi\left(x, v_{u}(x)\right) \mathrm{d} H^{n-1}(x),
$$

where $\phi$ is defined as in Theorem 1.1. Moreover, if $\mathcal{O}$ is Lipschitz, we have

$$
F^{\prime}(\boldsymbol{u}, \mathcal{O})=F^{\prime \prime}(\boldsymbol{u}, \mathcal{O})=G(\ell(\boldsymbol{u}), \mathcal{O}) .
$$

Proof. Let us assume that there exists the $\bar{\Gamma}$-limit as $\varepsilon \downarrow 0$ in $E$; by the representation result of Corollary 3.18, choosing

$$
\boldsymbol{u}:=\frac{1}{2}\left(\boldsymbol{s}_{-}+\boldsymbol{s}_{+}\right)+\frac{1}{2}\left(\boldsymbol{s}_{+}-\boldsymbol{s}_{-}\right) j\left(\left\langle x-x_{0}, v\right\rangle\right)
$$

we easily have

$$
\phi\left(x_{0}, \nu\right)=\lim _{\rho \downarrow 0} \frac{\bar{F}(\boldsymbol{u}, \rho Q)}{\rho^{n-1}} .
$$

Combining this asymptotic behaviour with the estimates of the previous lemma, we conclude.

\section{7 $\quad \Gamma$-convergence in the weak topology}

The aim of this section is to prove Theorem 1.2, by adapting the arguments of the previous paragraphs. Therefore, we fix a constant $C>0$, and we consider the topological space

$$
X_{w}:=\left\{\boldsymbol{u} \in L^{2}\left(\Omega ; \mathbb{R}^{m}\right):\|\boldsymbol{u}\|_{L^{2}\left(\Omega ; \mathbb{R}^{m}\right)} \leqslant C\right\}
$$


endowed with the coarsest topology which makes continuous the map

$$
\boldsymbol{u} \in X_{w} \rightarrow \ell(\boldsymbol{u}) \in L^{2}(\Omega) \text { with its weak topology. }
$$

In other words, as $\varepsilon \downarrow 0, \varepsilon \in E$

$$
\boldsymbol{u}_{\varepsilon} \rightarrow \boldsymbol{u} \text { in } X_{w} \quad \Leftrightarrow \quad u_{\varepsilon}:=\ell\left(\boldsymbol{u}_{\varepsilon}\right)-\ell(\boldsymbol{u})=u \text { in } L^{2}(\Omega) .
$$

It is not difficult to see that $X_{w}$ is not an Hausdorff space but nevertheless it satisfies the second countability axiom, i.e. it has a countable base of open sets. In fact, a set $Y \subset X_{w}$ is open iff $\ell(Y)$ is open in the weak topology of the ball of radius. $C|\ell|$ of $L^{2}(\Omega)$. Therefore, the definitions and the properties presented in Section 2.2-2.4 make sense also for this choice of $X_{w}$, and we can consider the family of local functionals $F, F^{\varepsilon}: X_{w} \times \boldsymbol{A}(\Omega) \rightarrow[0,+\infty], \varepsilon>0$, in this different setting.

Let us observe that a function $u \in L^{2}(\Omega)$ whose norm is bounded by $C \min _{i=1, \ldots, m}\left|\ell_{i}\right|^{-1}$ surely belongs to the image of $X_{w}$ through $\ell$; in particular, we are dealing in the limit with functions $u \in B V(\Omega ; T)$ which satisfy

$$
\|u\|_{L^{\infty}(\Omega)} \leqslant s_{\max }:=\max \left(\left|s_{-}\right|,\left|s_{+}\right|\right), \quad\|u\|_{L^{2}(\Omega)} \leqslant s_{\max }|\Omega|^{1 / 2},
$$

so that $B V(\Omega ; T) \subset \ell\left(X_{w}\right)$ whenever $C$ is sufficiently large.

Proof. In order to prove Theorem 1.2 we have simply to check that the various statements of Section 3 also hold with $X$ replaced by $X_{w}$. We limit ourselves to notice the relevant differences.

- Section 3.1: all these results do not deal with convergence, so they hold also in the new setting; in Lemma 3.4 we have only to specify that

$$
\|u\|_{L^{\infty}(\Omega)} \leqslant C|\Omega|^{-1 / 2} \min _{i=1, \ldots, m}\left|\ell_{i}\right|^{-1} .
$$

- Lemma 3.6 is already in the form we need; observe that if $\boldsymbol{u}^{\varepsilon}, \boldsymbol{v}^{\varepsilon} \in X_{w}$ then also $\boldsymbol{w}^{\varepsilon}$ belongs to $X_{w}$.

- Corollary 3.9 also holds, since if $\boldsymbol{u}^{\varepsilon}, \boldsymbol{v}^{\varepsilon} \rightarrow \boldsymbol{u}$ in $X_{w}$ then (3.15) is obviously satisfied and (3.16) can be deduced by the convergence in $X_{w}$ and the compactness provided by Lemma 3.2.

- Section 3.3 is not necessary in this context, since the topology on $X_{w}$ forces $F^{\prime}$ and $F^{\prime \prime}$ to depend only on $\ell(\boldsymbol{u})$, since they have to be lower semicontinuous in $X_{w}$.

- Section 3.4 and 3.5 hold without any change.

- Definition 3.19 makes sense also for $X_{w}$ and Proposition 3.21 is still true: we only have to observe that if we know only the weak convergence of $\ell\left(\boldsymbol{u}^{\varepsilon}\right)$ to $\ell(\boldsymbol{u})$ then the family $\boldsymbol{w}^{\varepsilon}$ defined in the proof of 3.21 converges to $u$ in $X_{w}$. In fact it is uniformly bounded by the constant $C$ in $L^{2}\left(\mathcal{O} ; \mathbb{R}^{m}\right)$ and, by construction,

$$
\boldsymbol{w}^{\varepsilon} \equiv \boldsymbol{u}^{\varepsilon} \quad \text { on every set } \mathcal{Q} \text { such that } \inf _{x \in \mathcal{Q}} d(x, \mathcal{O})>\varepsilon .
$$

Choosing test functions compactly supported in $\mathcal{O}$, we easily deduce the weak convergence of $\ell\left(\boldsymbol{w}^{\varepsilon}\right)$ to $\ell(\boldsymbol{u})$.

-Lemma 3.22, 3.23, and their $x$-dependent version hold without any change.

This concludes the proof in the weak convergence case. 


\section{4. $\Gamma$-convergence of the non-local functionals $G^{\varepsilon}$ for the 'bidomain' formulation.}

From now on we will assume that $F^{\varepsilon}$ have the particular 'bidomain structure' outlined in $\left(B_{1}-B_{6}\right)$, which we recall here for case of reference. Moreover we slightly modify our original notation in order to conform it to the previous section:

$$
\begin{gathered}
u_{1}:=u^{i}, A_{1}:=A^{i}, q_{1}:=q^{i}, \ldots ; \\
u_{2}:=-u^{e}, A_{2}:=A^{e}, q_{2}:=q^{e}, \ldots
\end{gathered}
$$

Therefore,

$\Omega \subset \mathbb{R}^{3}$ is the physical region occupied by the heart

$$
A_{1}, A_{2}: \bar{\Omega} \rightarrow \mathbb{M}^{3 \times 3} \text { are }
$$

symmetric, positive definite, continuous conductivity tensors,

$$
\boldsymbol{u}:=\left(u_{1}, u_{2}\right) \text { represents the couple }
$$

of the intra- and (the opposite of) the extracellular potentials,

$$
\begin{aligned}
F^{\varepsilon}(\boldsymbol{u}) & :=\varepsilon \int_{\Omega}\left(\left\langle A_{1} \nabla u_{1}, \nabla u_{1}\right\rangle+\left\langle A_{2} \nabla u_{2}, \nabla u_{2}\right\rangle\right) \mathrm{d} x \\
& +\frac{1}{\varepsilon} \int_{\Omega} W\left(u_{1}+u_{2}\right) \mathrm{d} x,
\end{aligned}
$$

$$
\ell(\boldsymbol{u}):=u_{1}+u_{2}=u \text {, defines the transmembrane potential, }
$$

and

$$
\begin{gathered}
q_{1,2}(x, \xi):=\left\langle A_{1,2}(x) \xi, \xi\right\rangle, \quad \forall \xi \in \mathbb{R}^{3}, \\
\boldsymbol{q}(x, \mathfrak{a}):=q_{1}\left(x, \mathfrak{a}_{1}\right)+q_{2}\left(x, \mathfrak{a}_{2}\right), \quad \mathfrak{a}=\left(\mathfrak{a}_{1}, \mathfrak{a}_{2}\right)^{T} \in \mathbb{M}^{2 \times 3} .
\end{gathered}
$$

\subsection{Dual a priori estimates for smooth coefficients}

We first consider the case of two matrices $A_{1}, A_{2}$ with Lipschitz coefficients, i.e.

$$
\exists L \geqslant 0: \quad\left|A_{i}(x)-A_{i}(y)\right| \leqslant L|x-y| \quad \forall x, y \in \bar{\Omega}, \quad i=1,2 .
$$

At the end of this section we will show how the results can be extended to continuous coefficients.

Let us first consider the minimum problem $(1.38)$ defining the functional $Q(\cdot, \mathcal{O}), \mathcal{O}$ being at least a Lipschitz and connected open subset of $\Omega$; we want to define a canonical choice among its minimizing solutions.

LEMma 4.1 For every $u \in H^{1}(\mathcal{O})$ there exists a unique element $\overline{\boldsymbol{u}}:=\overline{\boldsymbol{u}}(u, \mathcal{O}) \in H^{1}\left(\mathcal{O} ; \mathbb{R}^{2}\right)$ such that

$$
\begin{aligned}
\bar{u}_{1}+\bar{u}_{2}=u, \quad Q(u, \mathcal{O}) & =\int_{\mathcal{O}} q(x, \nabla \overline{\boldsymbol{u}}(x)) \mathrm{d} x, \text { and } \\
\int_{\mathcal{O}} \bar{u}_{1} \mathrm{~d} x & =\int_{\mathcal{O}} \bar{u}_{2} \mathrm{~d} x .
\end{aligned}
$$


It satisfies the equations (here $i^{\prime}:=2,1$ if respectively $i=1,2$ )

$$
\int_{\mathcal{O}}\left\langle A \nabla \bar{u}_{i}, \nabla w\right\rangle \mathrm{d} x=\int_{\mathcal{O}}\left\langle A_{i^{\prime}} \nabla u, \nabla w\right\rangle \mathrm{d} x \quad \forall w \in H^{1}(\mathcal{O}) .
$$

Moreover, if

$$
\mathcal{O} \text { is convex or of class } C^{1,1}
$$

there exists a constant $C>0$ (depending only on $\mathcal{O}, \lambda, \Lambda, L)$ such that

$$
\|\overline{\boldsymbol{u}}\|_{L^{2}\left(\mathcal{O} ; \mathbb{R}^{2}\right)} \leqslant C\left(\|u\|_{L^{2}(\mathcal{O})}+\left\|u_{\left.\right|_{\partial \mathcal{O}}}\right\|_{*}\right),
$$

where, for every $g \in L^{2}(\partial \mathcal{O})$

$$
\|g\|_{*}:=\sup \left\{\int_{\partial \mathcal{O}} g \partial_{\bar{n}} \zeta \mathrm{d} H^{n-1}: \zeta \in H^{2}(\mathcal{O}),\|\zeta\|_{H^{2}(\mathcal{O})} \leqslant 1\right\} .
$$

Proof. The existence of $\overline{\boldsymbol{u}}(u)^{\dagger}$ is a straightforward application in $H^{1}\left(\mathcal{O} ; \mathbb{R}^{2}\right)$ of the Poincaré inequality and of the Lions-Stampacchia Theorem on the closed affine subspace $K_{u}$ defined by

$$
K_{u}:=\left\{\boldsymbol{w} \in H^{1}\left(\mathcal{O} ; \mathbb{R}^{2}\right): w_{1}+w_{2}=u, \int_{\mathcal{O}} w_{1} \mathrm{~d} x=\int_{\mathcal{O}} w_{2} \mathrm{~d} x\right\}
$$

in fact, $\overline{\boldsymbol{u}}(u)$ is characterized by

$$
\overline{\boldsymbol{u}} \in K_{u}, \quad \sum_{i=1,2} \int_{\mathcal{O}}\left\langle A_{i} \nabla \bar{u}_{i}, \nabla w_{i}\right\rangle \mathrm{d} x=0 \quad \forall \boldsymbol{w} \in K_{0} .
$$

Since $\boldsymbol{w} \in K_{0} \Leftrightarrow w_{1}=-w_{2}, \quad \int_{\mathcal{O}_{k}} w_{1} \mathrm{~d} x=0$, we get

$$
\int_{\mathcal{O}}\left\langle A_{1} \nabla \bar{u}_{1}, \nabla w\right\rangle \mathrm{d} x=\int_{\mathcal{O}}\left\langle A_{2} \nabla \bar{u}_{2}, \nabla w\right\rangle \mathrm{d} x, \quad \forall w \in H^{1}(\mathcal{O}) .
$$

Finally, by adding $\int_{\mathcal{O}}\left\langle A_{2} \nabla \bar{u}_{1}, \nabla w\right\rangle \mathrm{d} x$ to both the members, we obtain (4.4) for $i=1$; the other equation can be derived analogously.

In order to prove the $L^{2}(\mathcal{O})$-estimate when $\mathcal{O}$ satisfies $(4.5)$, we use a standard duality argument. We set

$$
\bar{u}_{i}^{*}:=\bar{u}_{i}-\frac{1}{|\mathcal{O}|} \int_{\mathcal{O}} \bar{u}_{i}(x) \mathrm{d} x
$$

and we observe that the $L^{2}$-estimate of $\overline{\boldsymbol{u}}$ follows from (4.2),(4.3) if we are able to control the $L^{2}$-norms of $\bar{u}_{i}^{*}$.

So, let us consider the unique variational solution $\bar{w} \in H^{1}(\mathcal{O})$, with $\int_{\mathcal{O}} w(x) \mathrm{d} x=0$ (we assumed that $\mathcal{O}$ is connected), of the problem

$$
\int_{\mathcal{O}} A(x) \nabla \bar{w}(x) \nabla \zeta(x) \mathrm{d} x=\int_{\mathcal{O}} \bar{u}_{i}^{*}(x) \zeta(x) \mathrm{d} x, \quad \forall \zeta \in H^{1}(\Omega) .
$$

${ }^{\dagger}$ This does not require (4.1) but only (1.14ab). 
Since $\mathcal{O}$ is convex or $C^{1,1}$ and (4.1) holds, then it is well known that $\bar{w} \in H^{2}(\mathcal{O})$ and (see e.g. [20], Theorems 2.2.2.3 and 3.2.1.2)

$$
\|w\|_{H^{2}(\mathcal{O})} \leqslant C\left\|\bar{u}_{i}^{*}\right\|_{L^{2}(\Omega)} .
$$

Inserting (4.8) into (4.4) and choosing $\zeta:=\bar{u}_{i}$, we get after an integration by part

$$
\begin{aligned}
\int_{\mathcal{O}}\left|\bar{u}_{i}^{*}\right|^{2} \mathrm{~d} x= & \int_{\mathcal{O}}\left\langle A \nabla \bar{w} \nabla \bar{u}_{i}\right\rangle \mathrm{d} x=\int_{\mathcal{O}}\left\langle A_{i^{\prime}} \nabla u, \nabla \bar{w}\right\rangle \mathrm{d} x= \\
& -\int_{\mathcal{O}} u \mathcal{L}_{i^{\prime}} \bar{w} \mathrm{~d} x+\int_{\partial \mathcal{O}} u \partial_{\bar{n}^{\prime}} \bar{w} \mathrm{~d} H^{n-1} \leqslant C\left(\|u\|_{L^{2}(\mathcal{O})}+\left\|\left.u\right|_{\partial \mathcal{O}}\right\|_{*}\right)\|\bar{w}\|_{H^{2}(\Omega)} .
\end{aligned}
$$

By (4.9) we conclude.

\section{$4.2 \quad \Gamma$-limit of $G^{\varepsilon}$}

The previous estimates in the case (4.1) are useful to derive compactness properties for $G^{\varepsilon}$, defined by (1.39).

LEMmA 4.2 Let $\mathcal{O} \in \boldsymbol{A}(\Omega)$ be Lipschitz, let $\left\{u^{\varepsilon}\right\}_{\varepsilon>0}$ be a family in $L^{2}(\Omega)$ such that

$$
M:=\liminf _{\varepsilon \rightarrow 0^{+}} G^{\varepsilon}\left(u^{\varepsilon}, \mathcal{O}\right)<+\infty,
$$

and let $\overline{\boldsymbol{u}}^{\varepsilon}:=\overline{\boldsymbol{u}}\left(u^{\varepsilon}, \mathcal{O}\right)$. Then there exists an infinitesimal decreasing sequence $D \subset E$ and a function $u \in B V(\mathcal{O} ; T)$ such that

$$
\lim _{\varepsilon \downarrow 0, \varepsilon \in D} G^{\varepsilon}\left(u^{\varepsilon}, \mathcal{O}\right)=M, \quad \lim _{\varepsilon \downarrow 0, \varepsilon \in D} u^{\varepsilon}=u \text { in } L^{p_{0}}(\mathcal{O}),
$$

where $p_{0}$ is the growth exponent of $W$ defined in (1.15b). Moreover, if (4.5) holds, we can choose $D$ in such a way that

$$
\left.\lim _{\varepsilon \downarrow 0, \varepsilon \in D} u^{\varepsilon}\right|_{\partial \mathcal{O}}=g \text { weakly in } L^{2}(\partial \mathcal{O}) \text { and } \lim _{\varepsilon \downarrow 0, \varepsilon \in D} \overline{\boldsymbol{u}}^{\varepsilon}=\overline{\boldsymbol{u}} \text { in } L^{2}\left(\mathcal{O} ; \mathbb{R}^{2}\right)
$$

where $\overline{\boldsymbol{u}}$ solves $\left(i=1,2, i^{\prime}:=2,1\right.$ respectively)

$$
\int_{\mathcal{O}} \bar{u}_{i} \mathcal{L}(\zeta) \mathrm{d} x=\int_{\mathcal{O}} u \mathcal{L}_{i^{\prime}}(\zeta) \mathrm{d} x+\int_{\partial \mathcal{O}} g \partial_{\bar{n}_{i^{\prime}}} \zeta \mathrm{d} H^{n-1}(x),
$$

for every test function $\zeta \in H^{2}(\mathcal{O})$ with $\partial_{\bar{n}} \zeta=0$ on $\partial \mathcal{O}$.

Proof. The first part of the lemma follows directly by Lemma 3.2 since $G^{\varepsilon}\left(u^{\varepsilon}\right)=F^{\varepsilon}\left(\overline{\boldsymbol{u}}^{\varepsilon}\right)$. In order to show (4.12), we recall that, by the trace estimates for $W^{1,1}$-functions, the trace of $z^{\varepsilon}=$ $Z\left(u^{\varepsilon}\right), \varepsilon \in D$, (cf. (3.4),(3.6)) on $\partial \mathcal{O}$ are bounded in $L^{1}(\partial \mathcal{O})$; therefore,

$$
\left.u^{\varepsilon}\right|_{\partial \mathcal{O}} \text { are bounded in } L^{2}(\partial \mathcal{O}) \text { if } \varepsilon \in D .
$$

Up to a possible extraction of another subsequence, we deduce also the first relation of (4.12). It remains to show the second one, as (4.13) follows from (4.4) by a simple integration by parts.

Since $u^{\varepsilon}$ strongly converges to $u$ in $L^{2}(\mathcal{O})$ as $\varepsilon \downarrow 0, \varepsilon \in E$, the a priori estimate (4.6) and the next lemma yield the strong convergence of $\overline{\boldsymbol{u}}^{\varepsilon}$. 
LEMMA 4.3 Let us assume that $g^{\varepsilon} \rightarrow g$ in $L^{2}(\partial \mathcal{O})$ as $\varepsilon \downarrow 0, \varepsilon \in D$; then

$$
\lim _{\varepsilon \downarrow 0, \varepsilon \in D}\left\|g^{\varepsilon}-g\right\|_{*}=0 .
$$

Proof. Here the only difficulty arises when $\mathcal{O}$ is not $C^{1,1}$, so we can suppose it is a convex set. Now we fix $\delta \in(0,1 / 2)$ and we observe that, by the standard trace results, the map

$$
g \in L^{2}(\partial \mathcal{O}) \mapsto L_{g} \in\left(H^{2-\delta}(\mathcal{O})\right)^{\prime}, \quad\left\langle L_{g}, \zeta\right\rangle:=\int_{\partial \mathcal{O}} g \partial_{\overline{\boldsymbol{n}}_{i}} \zeta \mathrm{d} H^{n-1}
$$

is bounded and satisfies

$$
\|g\|_{*}=\left\|L_{g}\right\|_{\left(H^{2}(\mathcal{O})\right)^{\prime}} .
$$

On the other hand, $H^{2}(\Omega)$ is densely and compactly embedded into $H^{2-\delta}(\mathcal{O})$, so that the dual of $H^{2-\delta}(\mathcal{O})$ is compactly imbedded into the dual of $H^{2}(\mathcal{O})$. Since $L_{g^{\varepsilon}}$ weakly converge to $L_{g}$ in the first space, they also strongly converge in the second one; by (4.15), they converge w.r.t. the norm $\|\cdot\|_{*} \cdot$

COROLlary 4.4 If $\mathcal{O} \in \boldsymbol{A}(\Omega)$ is Lipschitz, then

$$
u^{\varepsilon} \rightarrow u \quad \text { in } D^{\prime}(\mathcal{O}) \quad \Rightarrow \quad \liminf _{\varepsilon \downarrow 0} G^{\varepsilon}\left(u^{\varepsilon}, \mathcal{O}\right) \geqslant G(u, \mathcal{O})
$$

Proof. We can assume $G(u, \mathcal{O})<+\infty$. By the previous compactness result, we can assume that $u^{\varepsilon} \rightarrow u$ in $L^{2}(\mathcal{O})$ and, if $\mathcal{O}$ is also of class $C^{1,1}$, we can find $\overline{\boldsymbol{u}}^{\varepsilon} \in L^{2}\left(\mathcal{O} ; \mathbb{R}^{2}\right)$ such that

$$
G^{\varepsilon}\left(u^{\varepsilon}, \mathcal{O}\right)=F^{\varepsilon}\left(\overline{\boldsymbol{u}}^{\varepsilon}, \mathcal{O}\right), \quad \overline{\boldsymbol{u}}^{\varepsilon} \rightarrow \overline{\boldsymbol{u}} \quad \text { in } L^{2}\left(\Omega ; \mathbb{R}^{2}\right), \quad \ell(\overline{\boldsymbol{u}})=u .
$$

Applying Theorem 1.1, we get

$$
\liminf _{\varepsilon \downarrow} F^{\varepsilon}\left(\overline{\boldsymbol{u}}^{\varepsilon}, \mathcal{O}\right) \geqslant F(\overline{\boldsymbol{u}}, \mathcal{O})=G(u, \mathcal{O}) .
$$

If $\mathcal{O}$ is only Lipschitz, we apply the previous result to all the open subsets $\mathcal{O}_{1} \Subset \mathcal{O}$ of class $C^{1,1}$, and we find

$$
\underset{\varepsilon \downarrow 0}{\liminf } G^{\varepsilon}\left(u^{\varepsilon}, \mathcal{O}\right) \geqslant \liminf _{\varepsilon \downarrow 0} G^{\varepsilon}\left(u^{\varepsilon}, \mathcal{O}_{1}\right) \geqslant G\left(u, \mathcal{O}_{1}\right)
$$

Taking the supremum w.r.t. $\mathcal{O}_{1}$, we conclude.

The proof of the first part of Theorem 1.5 and its corollary quickly follows: by the previous corollary, we know that for every Lipschitz open set $\mathcal{O}$

$$
G^{\prime}(u, \mathcal{O}) \geqslant G(u, \mathcal{O})=F(\boldsymbol{u}, \mathcal{O}) \quad \forall \boldsymbol{u}: \ell(\boldsymbol{u})=u
$$

on the other hand, as

$$
G^{\varepsilon}(u, \mathcal{O}) \leqslant F^{\varepsilon}(\boldsymbol{u}, \mathcal{O}) \quad \text { whenever } u=\ell(\boldsymbol{u}),
$$

it is easy to see that

$$
G^{\prime \prime}(u, \mathcal{O}) \leqslant \inf \left\{F^{\prime \prime}(\boldsymbol{u}, \mathcal{O}): \ell(\boldsymbol{u})=u\right\}=F(\boldsymbol{u}, \mathcal{O})=G^{\prime}(u, \mathcal{O})
$$




\subsection{Strong convergence of the traces and the potentials for optimal sequences}

In order to conclude the proof of the second part of Theorem 1.5 we need the following two Lemmata.

LEMmA 4.5 Let $\boldsymbol{u}^{\varepsilon}$ be a bounded family in $L^{2}\left(\Omega ; \mathbb{R}^{2}\right)$ satisfying

$$
\ell\left(\boldsymbol{u}^{\varepsilon}\right) \rightarrow u \text { in } L^{2}(\Omega), \quad \limsup _{\varepsilon \downarrow 0} F^{\varepsilon}\left(\boldsymbol{u}^{\varepsilon}, \Omega\right) \leqslant G(u, \Omega) .
$$

Then for every Lipschitz open set $\mathcal{O} \Subset \Omega$ we have

$$
\begin{gathered}
\limsup _{\varepsilon \downarrow 0} F^{\varepsilon}\left(\boldsymbol{u}^{\varepsilon}, \mathcal{O}\right) \leqslant \inf \left\{G\left(u, \mathcal{O}^{\prime}\right): \mathcal{O} \Subset \mathcal{O}^{\prime}\right\}, \\
\limsup _{\varepsilon \downarrow 0} F^{\varepsilon}\left(\boldsymbol{u}^{\varepsilon}, \Omega \backslash \overline{\mathcal{O}}\right) \leqslant \inf \left\{G\left(u, \Omega \backslash \overline{\mathcal{O}^{\prime \prime}}\right): \mathcal{O}^{\prime \prime} \Subset \mathcal{O}\right\} .
\end{gathered}
$$

In particular, recalling the definition of $\Omega_{\rho}$ given by (2.15), we have

$$
\lim _{\rho \downarrow 0} \limsup _{\varepsilon \downarrow 0} F^{\varepsilon}\left(\boldsymbol{u}^{\varepsilon}, \Omega_{\rho}\right)=0 .
$$

Proof. Let us only check (4.16b), since the first formula follows by the same arguments.

Let us choose $\mathcal{O}_{1}, \mathcal{O}_{2} \in A(\Omega)$ such that

$$
\mathcal{O}_{1} \Subset \mathcal{O}_{2} \Subset \mathcal{O} .
$$

We easily have

$$
\begin{aligned}
\limsup _{\varepsilon \downarrow 0} F^{\varepsilon}\left(\boldsymbol{u}^{\varepsilon}, \Omega \backslash \overline{\mathcal{O}}\right)+G\left(u, \mathcal{O}_{1}\right) & \leqslant \limsup _{\varepsilon \downarrow 0} F^{\varepsilon}\left(\boldsymbol{u}^{\varepsilon}, \Omega \backslash \overline{\mathcal{O}}\right)+\liminf _{\varepsilon \downarrow 0} F^{\varepsilon}\left(\boldsymbol{u}^{\varepsilon}, \mathcal{O}_{1}\right) \\
& \leqslant \limsup _{\varepsilon \downarrow 0} F^{\varepsilon}\left(\boldsymbol{u}^{\varepsilon},(\Omega \backslash \mathcal{O}) \cup \mathcal{O}_{1}\right) \\
& \leqslant \limsup _{\varepsilon \downarrow 0} F^{\varepsilon}\left(\boldsymbol{u}^{\varepsilon}, \Omega\right) \\
& \leqslant G(u, \Omega) .
\end{aligned}
$$

From these inequalities we get

$$
\underset{\varepsilon \downarrow 0}{\limsup } F^{\varepsilon}\left(\boldsymbol{u}^{\varepsilon}, \Omega \backslash \overline{\mathcal{O}}\right) \leqslant G(u, \Omega)-G\left(u, \mathcal{O}_{1}\right) \leqslant G\left(u, \Omega \backslash \overline{\mathcal{O}_{2}}\right) .
$$

As $\mathcal{O}_{2} \Subset \mathcal{O}$ is arbitrary, we obtain (4.16b).

In order to prove (4.17), we call

$$
\Omega_{\rho}^{\prime}:=\{x \in \Omega: \mathrm{d}(x, \partial \Omega)>\rho\}, \quad \Omega_{\rho}=\Omega \backslash \bar{\Omega}_{\rho}^{\prime}
$$

and we observe that if $\rho, \sigma$ are sufficiently small,

$$
\rho<\sigma \Rightarrow \Omega_{\sigma}^{\prime} \Subset \Omega_{\rho}^{\prime} ;
$$


by the previous formula

$$
\limsup _{\varepsilon \downarrow 0} F^{\varepsilon}\left(\boldsymbol{u}^{\varepsilon}, \Omega_{\rho}\right) \leqslant G\left(u, \Omega_{\sigma}\right), \quad \forall \sigma>\rho>0
$$

and finally

$$
\lim _{\rho \downarrow 0} \limsup _{\varepsilon \downarrow 0} F^{\varepsilon}\left(\boldsymbol{u}^{\varepsilon}, \Omega_{\rho}\right) \leqslant \lim _{\sigma \downarrow 0} G\left(u, \Omega_{\sigma}\right)=0 .
$$

LEMMA 4.6 Let $\Omega$ be of class $C^{1,1}$ (or convex) and let $u^{\varepsilon}$ be a family in $L^{2}(\Omega)$ satisfying (1.46). Then the traces of $u^{\varepsilon}$ on $\partial \Omega$ strongly converge to the trace of $u$ in $L^{2}(\partial \Omega)$.

Proof. We already know that the traces of $u^{\varepsilon}$ are uniformly bounded in $L^{2}(\partial \Omega)$. We introduce the auxiliary functions $z^{\varepsilon}:=Z\left(u^{\varepsilon}\right)$ (cf. (3.4) and we observe that, for every $\rho>0$

$$
\int_{\Omega_{\rho}}\left|\nabla z^{\varepsilon}\right| \mathrm{d} x \leqslant \frac{|\ell|}{\sqrt{\lambda}} F^{\varepsilon}\left(\overline{\boldsymbol{u}}^{\varepsilon}, \Omega_{\rho}\right)
$$

where $\overline{\boldsymbol{u}}^{\varepsilon}:=\overline{\boldsymbol{u}}^{\varepsilon}\left(u^{\varepsilon}, \Omega\right)$ are defined from $u^{\varepsilon}$ as in Lemma 4.1. By applying the previous lemma, we get

$$
\lim _{\rho \downarrow 0} \limsup \int_{\varepsilon \downarrow 0}\left|\nabla z^{\varepsilon}\right| \mathrm{d} x \leqslant \frac{|\ell|}{\sqrt{\lambda}} \lim _{\rho \downarrow 0} \limsup _{\varepsilon \downarrow 0} F^{\varepsilon}\left(\overline{\boldsymbol{u}}^{\varepsilon}, \Omega_{\rho}\right)=0 .
$$

Lemma 2.1 therefore entails

$$
\left.z^{\varepsilon}\right|_{\partial \Omega} \rightarrow z_{\partial \Omega} \text { in } L^{1}(\partial \Omega)
$$

By (3.5) and (3.6), we deduce that the inverse function $Z^{-1}$ of $Z$ can be bounded by

$$
\left|Z^{-1}(s)\right| \leqslant c_{2} \sqrt{1+|s|}, \quad \text { for some constant } c_{2}>0 .
$$

Therefore

$$
u_{\left.\varepsilon\right|_{\partial \Omega}}=\left.Z^{-1}\left(z^{\varepsilon}\right)_{\left.\right|_{\partial} \Omega} \rightarrow Z^{-1}(z)\right|_{\partial \Omega} \quad \text { in } L^{2}(\partial \Omega)
$$

and it is obvious that

$$
Z^{-1}(z)_{\left.\right|_{\partial \Omega}}=u_{\left.\right|_{\partial \Omega}} .
$$

The final part of Theorem 1.5 follows now by another application of Lemma 4.2. 


\section{$4.4 \quad \Gamma$-convergence of $G^{\varepsilon}$ in the case of continuous coefficients}

When the matrix-valued functions $A_{1}, A_{2}: \bar{\Omega} \rightarrow \mathbb{M}^{3 \times 3}$ are only continuous, the dual a priori estimate (4.6) is no longer true, in general, and the weak formulation $(1.47 \mathrm{a}, \mathrm{b})$ does not make sense. However, we can prove the $\Gamma$-convergence of $G^{\varepsilon}$ to $G$ by a simple approximation result.

First of all, we define the distance between two continuous and positive definite quadratic forms $\boldsymbol{q}_{1}, \boldsymbol{q}_{2}$ satisfying $(1.14 \mathrm{a}, \mathrm{b})$ by

$$
\mathrm{d}\left(\boldsymbol{q}_{1}, \boldsymbol{q}_{2}\right):=\sup _{x \in \Omega, \mathfrak{a} \in \mathbb{M}^{m \times n} \backslash\{0\}} \frac{\left|\boldsymbol{q}_{1}(x, \mathfrak{a})-\boldsymbol{q}_{2}(x, \mathfrak{a})\right|}{|\mathfrak{a}|^{2}} .
$$

The basic fact is contained in the following lemma.

Lemma 4.7 For $i=1,2$ let $\boldsymbol{q}_{i}$ be satisfying $(1.14 \mathrm{a}, \mathrm{b})$ and let $F_{i}^{\varepsilon}, F_{i}$ be the corresponding functionals defined by (1.17) and Theorem 1.1. Then for every $\boldsymbol{v} \in L^{2}\left(\Omega ; \mathbb{R}^{2}\right)$ we have

$$
\begin{gathered}
\left|F_{1}^{\varepsilon}(\boldsymbol{v})-F_{2}^{\varepsilon}(\boldsymbol{v})\right| \leqslant \frac{\mathrm{d}\left(\boldsymbol{q}_{1}, \boldsymbol{q}_{2}\right)}{\lambda} \min \left\{F_{1}^{\varepsilon}(\boldsymbol{v}), F_{2}^{\varepsilon}(\boldsymbol{v})\right\}, \\
\left|F_{1}(\boldsymbol{v})-F_{2}(\boldsymbol{v})\right| \leqslant \frac{\mathrm{d}\left(\boldsymbol{q}_{1}, \boldsymbol{q}_{2}\right)}{\lambda} \max \left\{F_{1}(\boldsymbol{v}), F_{2}(\boldsymbol{v})\right\} .
\end{gathered}
$$

Proof. The first inequality follows easily by

$$
\begin{aligned}
\left|F_{1}^{\varepsilon}(\boldsymbol{v})-F_{2}^{\varepsilon}(\boldsymbol{v})\right| & \leqslant \varepsilon\left|\int_{\Omega}\left(\boldsymbol{q}_{1}(x, \nabla \boldsymbol{v}(x))-\boldsymbol{q}_{2}(x, \nabla \boldsymbol{v}(x))\right) \mathrm{d} x\right| \\
& \leqslant \varepsilon \mathrm{d}\left(\boldsymbol{q}_{1}, \boldsymbol{q}_{2}\right) \int_{\Omega}|\nabla \boldsymbol{v}(x)|^{2} \mathrm{~d} x \\
& \leqslant \frac{\mathrm{d}\left(\boldsymbol{q}_{1}, \boldsymbol{q}_{2}\right)}{\lambda} \min \left\{F_{1}^{\varepsilon}(\boldsymbol{v}), F_{2}^{\varepsilon}(\boldsymbol{v})\right\} .
\end{aligned}
$$

In order to check the other one, we take a family $\boldsymbol{v}^{\varepsilon} \in L^{2}\left(\Omega ; \mathbb{R}^{2}\right)$ such that

$$
\boldsymbol{v}^{\varepsilon} \rightarrow \boldsymbol{v} \quad \text { in } L^{2}\left(\Omega ; \mathbb{R}^{2}\right), \quad \lim _{\varepsilon \downarrow 0} F_{1}^{\varepsilon}\left(\boldsymbol{v}^{\varepsilon}\right)=F_{1}(\boldsymbol{v}) .
$$

We obtain

$$
\begin{aligned}
F_{2}(\boldsymbol{v})-F_{1}(\boldsymbol{v}) & \leqslant \liminf _{\varepsilon \downarrow 0}\left(F_{2}^{\varepsilon}\left(\boldsymbol{v}^{\varepsilon}\right)-F_{1}^{\varepsilon}\left(\boldsymbol{v}^{\varepsilon}\right)\right) \\
& \leqslant \frac{\mathrm{d}\left(\boldsymbol{q}_{1}, \boldsymbol{q}_{2}\right)}{\lambda} \liminf _{\varepsilon \downarrow 0} \min \left\{F_{1}^{\varepsilon}\left(\boldsymbol{v}^{\varepsilon}\right), F_{2}^{\varepsilon}\left(\boldsymbol{v}^{\varepsilon}\right)\right\} \\
& \leqslant \frac{\mathrm{d}\left(\boldsymbol{q}_{1}, \boldsymbol{q}_{2}\right)}{\lambda} F_{1}(\boldsymbol{v}) .
\end{aligned}
$$

Changing the role of $F_{1}$ and $F_{2}$ we conclude.

We can now complete the last step of the proof of Theorem 1.5 for a continuous family of quadratic forms. 
LEMMA 4.8 Let $\boldsymbol{q}$ satisfy (1.14a, b) and let $G^{\varepsilon}$ be defined as in (1.39); if

$$
u^{\varepsilon} \rightarrow u \quad \text { in } L^{2}(\Omega)
$$

then

$$
\liminf _{\varepsilon \downarrow 0} G^{\varepsilon}\left(u^{\varepsilon}\right) \geqslant G(u) .
$$

Proof. It is not restrictive to assume that

$$
\limsup _{\varepsilon \downarrow 0} G^{\varepsilon}\left(u^{\varepsilon}\right)=M<+\infty .
$$

Let $\overline{\boldsymbol{u}}^{\varepsilon}=\overline{\boldsymbol{u}}\left(u^{\varepsilon}, \Omega\right)$ be defined as in Lemma 4.1 so that

$$
G^{\varepsilon}\left(u^{\varepsilon}\right)=F^{\varepsilon}\left(\overline{\boldsymbol{u}}^{\varepsilon}\right)
$$

and let $\boldsymbol{q}_{*}: \bar{\Omega} \times \mathbb{M}^{2 \times 3} \rightarrow \mathbb{R}$ be another family of quadratic forms with Lipschitz coefficients satisfying (1.14aa, b) and (4.1). We denote by $F_{*}^{\varepsilon}, G_{*}^{\varepsilon}$ the corresponding functionals defined by $(1.17),(1.39)$ respectively, and by $F_{*}, G_{*}$ their $\Gamma$-limits as in Theorems $1.1,1.2$ and 1.5; we also take

$$
\overline{\boldsymbol{u}}_{*} \in L^{2}\left(\Omega ; \mathbb{R}^{2}\right) \quad \text { such that } \ell\left(\boldsymbol{u}_{*}\right)=u, \quad G_{*}(u)=F_{*}\left(\overline{\boldsymbol{u}}_{*}\right)
$$

Applying the previous lemma we get

$$
\begin{aligned}
G^{\varepsilon}\left(u^{\varepsilon}\right) & =G^{\varepsilon}\left(u^{\varepsilon}\right)-G_{*}^{\varepsilon}\left(u^{\varepsilon}\right)+G_{*}^{\varepsilon}\left(u^{\varepsilon}\right) \\
& \geqslant F^{\varepsilon}\left(\overline{\boldsymbol{u}}^{\varepsilon}\right)-F_{*}^{\varepsilon}\left(\overline{\boldsymbol{u}}^{\varepsilon}\right)+G_{*}^{\varepsilon}\left(u^{\varepsilon}\right) \\
& \geqslant-\mathrm{d}\left(\boldsymbol{q}, \boldsymbol{q}_{*}\right) F^{\varepsilon}\left(\overline{\boldsymbol{u}}^{\varepsilon}\right)+G_{*}^{\varepsilon}\left(u^{\varepsilon}\right) \\
& =-\mathrm{d}\left(\boldsymbol{q}, \boldsymbol{q}_{*}\right) G^{\varepsilon}\left(u^{\varepsilon}\right)+G_{*}^{\varepsilon}\left(u^{\varepsilon}\right) .
\end{aligned}
$$

Passing to the limit as $\varepsilon \downarrow 0$ we get

$$
\begin{aligned}
\liminf _{\varepsilon \downarrow 0} G^{\varepsilon}\left(u^{\varepsilon}\right) & \geqslant G_{*}(u)-M \mathrm{~d}\left(\boldsymbol{q}, \boldsymbol{q}_{*}\right) \\
& =F\left(\overline{\boldsymbol{u}}_{*}\right)+\left(G_{*}(u)-F\left(\overline{\boldsymbol{u}}_{*}\right)\right)-M \mathrm{~d}\left(\boldsymbol{q}, \boldsymbol{q}_{*}\right) \\
& =G(u)+\left(F_{*}\left(\overline{\boldsymbol{u}}_{*}\right)-F\left(\overline{\boldsymbol{u}}_{*}\right)\right)-M \mathrm{~d}\left(\boldsymbol{q}, \boldsymbol{q}_{*}\right) \\
& \geqslant G(u)-\mathrm{d}\left(\boldsymbol{q}, \boldsymbol{q}_{*}\right) \max \left\{F_{*}\left(\overline{\boldsymbol{u}}_{*}\right), F\left(\overline{\boldsymbol{u}}_{*}\right)\right\}-M \mathrm{~d}\left(\boldsymbol{q}, \boldsymbol{q}_{*}\right) \\
& \geqslant G(u)\left(1-C \mathrm{~d}\left(\boldsymbol{q}, \boldsymbol{q}_{*}\right)\right)
\end{aligned}
$$

where $C$ is a positive constant depending on $G(u), \lambda, \Lambda$. Since by standard approximation results we can find a Lipschitz continuous family of quadratic forms $\boldsymbol{q}_{*}$ arbitrarily close to $\boldsymbol{q}$ w.r.t. the distance $d$, we conclude.

\section{A strict estimate from below.}

This last section contains the proof of Theorem 1.8 for the 'bidomain model'. 
As we have seen in Section 3, it is not restrictive to assume $A_{1}, A_{2}$ independent of the space variable $x$. In the next lemma, we show a simple but important algebraic relation between $\boldsymbol{q}$ and $q$. Let us recall that $\underline{A}$ is the symmetric and positive definite matrix

$$
\underline{A}=A_{1} A^{-1} A_{2}=A_{2} A^{-1} A_{1}=\left(A_{1}^{-1}+A_{2}^{-1}\right)^{-1} .
$$

Lemma 5.1 Let $A_{1}, A_{2}, A:=A_{1}+A_{2}$ be symmetric and definite positive matrices; let $\boldsymbol{q}, q$ and $\underline{q}$ be the quadratic forms

$$
\begin{gathered}
\boldsymbol{q}(\boldsymbol{\xi}):=\left\langle A_{1} \xi_{1}, \xi_{1}\right\rangle+\left\langle A_{2} \xi_{2}, \xi_{2}\right\rangle \quad \forall \xi=\left(\xi_{1}, \xi_{2}\right) \in \mathbb{R}^{n} \times \mathbb{R}^{n} \\
q(\xi)=\langle A \xi, \xi\rangle, \quad \underline{q}(\xi):=\langle\underline{A} \xi, \xi\rangle \quad \forall \xi \in \mathbb{R}^{n} .
\end{gathered}
$$

If $R_{i}:=A^{-1} A_{i}$, then for every choice of $\xi \in \mathbb{R}^{n} \times \mathbb{R}^{n}, \xi:=\xi_{1}+\xi_{2}$, we have

$$
\boldsymbol{q}(\boldsymbol{\xi})=\underline{q}(\xi)+q\left(R_{1} \xi_{1}-R_{2} \xi_{2}\right) .
$$

Proof. We simply have

$$
\begin{aligned}
\boldsymbol{q}(\boldsymbol{\xi})-\underline{q}(\xi) & =\left\langle A_{1} \xi_{1}, \xi_{1}\right\rangle+\left\langle A_{2} \xi_{2}, \xi_{2}\right\rangle-\left\langle\underline{A}\left(\xi_{1}+\xi_{2}\right), \xi_{1}+\xi_{2}\right\rangle \\
& =\left\langle\left(A_{1}-\underline{A}\right) \xi_{1}, \xi_{1}\right\rangle+\left\langle\left(A_{2}-\underline{A}\right) \xi_{2}, \xi_{2}\right\rangle-2\left\langle\underline{A} \xi_{1}, \xi_{2}\right\rangle .
\end{aligned}
$$

We observe that

$$
\begin{aligned}
A_{1}-\underline{A} & =A_{1}-A_{1} A^{-1} A_{2}=A_{1} A^{-1} A-A_{1} A^{-1} A_{2} \\
& =A_{1} A^{-1}\left(A-A_{2}\right)=A_{1} A^{-1} A_{1},
\end{aligned}
$$

and analogously

$$
A_{2}-\underline{A}=A_{2} A^{-1} A_{2} .
$$

Substituting into the previous equation we get

$$
\boldsymbol{q}(\boldsymbol{\xi})-\underline{q}(\xi)=\left\langle A_{1} A^{-1} A_{1} \xi_{1}, \xi_{1}\right\rangle+\left\langle A_{2} A^{-1} A_{2} \xi_{2}, \xi_{2}\right\rangle-2\left\langle\underline{A} \xi_{1}, \xi_{2}\right\rangle .
$$

Now we observe that

$$
\begin{aligned}
\left\langle A_{1} A^{-1} A_{1} \xi_{1}, \xi_{1}\right\rangle & =\left\langle\left(A_{1} A^{-1}\right) A\left(A^{-1} A_{1}\right) \xi_{1}, \xi_{1}\right\rangle \\
& =\left\langle A\left(A^{-1} A_{1}\right) \xi_{1},\left(A^{-1} A_{1}\right) \xi_{1}\right\rangle=q\left(R_{1} \xi_{1}\right),
\end{aligned}
$$

and

$\left\langle A_{2} A^{-1} A_{2} \xi_{2}, \xi_{2}\right\rangle=q\left(R_{2} \xi_{2}\right),\left\langle\underline{A} \xi_{1}, \xi_{2}\right\rangle=\left\langle\underline{A} \xi_{2}, \xi_{1}\right\rangle=\left\langle\left(A_{1} A^{-1}\right) A\left(A^{-1} A_{2}\right) \xi_{2}, \xi_{1}\right\rangle=\left\langle A R_{2} \xi_{2}, R_{1} \xi_{1}\right\rangle$.

Combining these three relations we get the thesis.

Let us now fix a direction $v \in \mathbb{S}^{n-1}$ and an admissible cylinder $Q=\Sigma(a, b ; \eta)$ w.r.t. $v$, as in definition (1.20a-e); we will also introduce the corresponding infinitely stretched cylinders

$$
Q^{\infty}:=\Sigma(-\infty, \infty ; \eta), \quad Q_{+}^{\infty}:=\Sigma(0, \infty ; \eta), \quad Q_{-}^{\infty}:=\Sigma(-\infty, 0 ; \eta) .
$$


Since we are dealing with unbounded domains, we modify slightly the definition of $\mathcal{B}\left(Q ; \mathbb{R}^{2}\right)$ as

$$
\begin{gathered}
\mathcal{C}\left(Q^{\infty} ; \mathbb{R}^{2}\right):=\left\{\boldsymbol{u} \in H_{l o c}^{1}\left(Q^{\infty} ; \mathbb{R}^{2}\right): \int_{Q^{\infty}}|\nabla \boldsymbol{u}(x)|^{2} \mathrm{~d} x<+\infty\right. \\
\left.\exists C>0: u(x \pm t \eta) \equiv s_{ \pm} \text {if } x \in \Sigma, t \geqslant C\right\}
\end{gathered}
$$

LEMMA 5.2 Let $\phi: \mathbb{S}^{n-1} \rightarrow \mathbb{R}$ be the function defined as in (1.25); then there exists a family $\boldsymbol{u}^{k} \in \mathcal{C}\left(Q^{\infty} ; \mathbb{R}^{2}\right), u^{k}=u_{1}^{k}+u_{2}^{k}$, such that

$$
\phi(v) \geqslant \limsup _{k \rightarrow+\infty} \int_{Q^{\infty}}\left(\boldsymbol{q}\left(\nabla \boldsymbol{v}^{k}(x)\right)+W\left(v^{k}(x)\right)\right) \mathrm{d} x,
$$

and, for $u^{k}:=Z\left(u^{k}\right)(\mathrm{cf} .(3.4)$

$$
\int_{Q_{-}^{\infty}} \sqrt{\underline{q}\left(\nabla z^{k}\right)} \mathrm{d} x=\int_{Q_{+}^{\infty}} \sqrt{\underline{q}\left(\nabla z^{k}\right)} \mathrm{d} x \quad \forall k \in \mathbb{N} .
$$

Proof. From Theorem 1.1 we know that there exists a family of functions $\boldsymbol{v}^{\varepsilon} \in \mathcal{B}\left(Q ; \mathbb{R}^{2}\right), \varepsilon>0$, such that

$$
\phi(v)=\lim _{\varepsilon \downarrow 0} \varepsilon \int_{Q} \boldsymbol{q}\left(\boldsymbol{v}^{\varepsilon}(x)\right) \mathrm{d} x+\frac{1}{\varepsilon} \int_{Q} W\left(v^{\varepsilon}(x)\right) \mathrm{d} x .
$$

Let $\boldsymbol{s}_{ \pm}^{\varepsilon}$ be the (constant) traces of $\boldsymbol{v}^{\varepsilon}$ on the basis $\Sigma_{ \pm}$of the cylinder $Q$; by definition, the sum of their coordinates is exactly $s_{ \pm}$. We extend $v^{\varepsilon}$ to the whole $Q^{\infty}$ by setting

$$
\boldsymbol{v}^{\varepsilon}(x)= \begin{cases}\boldsymbol{s}_{-}^{\varepsilon} & \text { if } x \in Q_{-}^{\infty} \backslash Q, \\ \boldsymbol{s}_{+}^{\varepsilon} & \text { if } x \in Q_{+}^{\infty} \backslash Q .\end{cases}
$$

It is easy to see that $\boldsymbol{v}^{\varepsilon} \in \mathcal{C}\left(Q^{\infty} ; \mathbb{R}^{2}\right)$. We set

$$
\boldsymbol{w}^{\varepsilon}(y):=\boldsymbol{v}^{\varepsilon}(\varepsilon y), \quad \forall y \in \varepsilon^{-1} Q^{\infty},
$$

so that $\nabla \boldsymbol{w}^{\varepsilon}(x)=\varepsilon \nabla \boldsymbol{v}^{\varepsilon}(\varepsilon x)$. We easily find

$$
\begin{aligned}
& \varepsilon \int_{Q^{\infty}} \boldsymbol{q}\left(\nabla \boldsymbol{v}^{\varepsilon}(x)\right) \mathrm{d} x+\frac{1}{\varepsilon} \int_{Q^{\infty}} W\left(v^{\varepsilon}(x)\right) \mathrm{d} x= \\
& \varepsilon^{n+1} \int_{\varepsilon^{-1} Q^{\infty}} \boldsymbol{q}\left(\nabla \boldsymbol{v}^{\varepsilon}(\varepsilon y)\right) \mathrm{d} y+\varepsilon^{n-1} \int_{\varepsilon^{-1} Q^{\infty}} W\left(v^{\varepsilon}(\varepsilon y)\right) \mathrm{d} y= \\
& \varepsilon^{n-1}\left(\int_{\varepsilon^{-1} Q^{\infty}} \boldsymbol{q}\left(\nabla \boldsymbol{w}^{\varepsilon}(y)\right) \mathrm{d} y+\int_{\varepsilon^{-1} Q^{\infty}} W\left(w^{\varepsilon}(y)\right) \mathrm{d} y\right) .
\end{aligned}
$$

Now we choose $\varepsilon:=(2 k+1)^{-1}, k \in \mathbb{N}$, and we observe that $\varepsilon^{-1} Q^{\infty}=(2 k+1) Q^{\infty}$ is the disjoint union (up to a negligible set) of

$$
(2 k+1) Q^{\infty}=\bigcup_{\max \left|j_{m}\right| \leqslant k}\left(Q^{\infty}+\sum_{m=1}^{n-1} j_{m} \eta_{m}\right) .
$$


Therefore there exist integers $j_{1}^{*}, j_{2}^{*}, \ldots, j_{n-1}^{*}$ between $-k$ and $k$ and a cylinder

$$
Q^{*}:=Q^{\infty}+\sum_{m=1}^{n-1} j_{m}^{*} \eta_{m}
$$

such that

$$
\begin{gathered}
\varepsilon^{n-1}\left(\int_{\varepsilon^{-1} Q^{\infty}} \boldsymbol{q}\left(\nabla \boldsymbol{w}^{\varepsilon}(y)\right) \mathrm{d} y+\int_{\varepsilon^{-1} Q^{\infty}} W\left(w^{\varepsilon}(y)\right) \mathrm{d} y\right) \\
\geqslant\left(\int_{Q^{*}} \boldsymbol{q}\left(\nabla \boldsymbol{w}^{\varepsilon}(y)\right) \mathrm{d} y+\int_{Q^{*}} W\left(w^{\varepsilon}(y)\right) \mathrm{d} y\right) .
\end{gathered}
$$

Finally we set

$$
\tilde{\boldsymbol{u}}^{k}(x):=\boldsymbol{w}^{\varepsilon}\left(x-\sum_{m=1}^{n-1} j_{m}^{*} \eta_{m}\right)
$$

which satisfy (5.4), i.e.

$$
\phi(v) \geqslant \limsup _{k \rightarrow+\infty} \int_{Q^{\infty}}\left(\boldsymbol{q}\left(\nabla \tilde{\boldsymbol{u}}^{k}(x)\right)+W\left(\tilde{u}^{k}(x)\right)\right) \mathrm{d} x .
$$

Now we have only to modify $\tilde{\boldsymbol{u}}^{k}$ in order to satisfy (5.5) too. Let us remark that, by (3.9), $\tilde{z}^{k}:=$ $Z\left(\tilde{u}^{k}\right) \in W_{l o c}^{1,1}\left(Q^{\infty}\right)$ and

$$
\int_{Q^{\infty}} \sqrt{\left.\underline{q\left(\nabla \tilde{z}^{k}\right.}\right)} \mathrm{d} x \leqslant \int_{Q^{\infty}}\left(\boldsymbol{q}\left(\nabla \tilde{\boldsymbol{u}}^{k}(x)\right)+W\left(\tilde{u}^{k}(x)\right)\right) \mathrm{d} x .
$$

We introduce the function

$$
\beta^{k}(s):=\int_{-\infty}^{s}\left(\int_{\Sigma} \sqrt{\underline{q\left(\nabla \tilde{z}^{k}(x+s \eta)\right)}} \mathrm{d} H^{n-1}(x)\right) \mathrm{d} s
$$

which is continuous, monotone and satisfies

$$
\beta^{k}(-\infty)=0, \quad \beta^{k}(+\infty)=|\langle\eta, v\rangle| \int_{Q^{\infty}} \sqrt{\underline{q}\left(\nabla \tilde{z}^{k}\right)} \mathrm{d} x .
$$

It is obvious that there exists real numbers $s^{k}$ such that $\beta^{k}\left(s^{k}\right)=\frac{\beta^{k}(+\infty)}{2}$; choosing $\boldsymbol{u}^{k}(x):=$ $\tilde{\boldsymbol{u}}^{k}\left(x+s^{k} \eta\right)$ we conclude.

COROLlary 5.3 Let $\phi: \mathbb{S}^{n-1} \rightarrow \mathbb{R}$ be the function defined as in (1.25), $v \in \mathbb{S}^{n-1}$, and $Q^{\infty}$ be an admissible infinite cylinder for $v$. Then

$$
\phi(v) \geqslant I(v):=\inf \left\{\int_{Q^{\infty}} \boldsymbol{q}(\nabla \boldsymbol{u}(x))+W(u(x)) \mathrm{d} x: \boldsymbol{u} \in \mathcal{C}\left(Q^{\infty} ; \mathbb{R}^{2}\right)\right\} .
$$


In order to conclude the proof of Theorem 1.8, we want to show that if $\phi(v)=\phi(v)$, then $v$ is a common eigenvector of $A_{1}, A_{2}$. Let us denote by $\|\cdot\|_{q}$ the norm associated to $q$,

$$
\|\xi\|_{\underline{q}}:=\sqrt{\underline{q}(\xi)} .
$$

Let us choose $\eta$ as

$$
\eta:=\frac{\underline{A} v}{\underline{q}(v)}, \quad \text { so that }\langle\eta, v\rangle=1,
$$

and let us consider $\underline{q}$-projection along $v$

$$
\Pi \xi:=\frac{\langle\underline{A} \xi, v\rangle}{\underline{q}(v)} v=\langle\xi, \eta\rangle \nu, \quad \Pi^{\prime} \xi:=\xi-\Pi \xi .
$$

Of course we have

$$
\underline{q}(\xi)=\underline{q}\left(\Pi^{\prime} \xi\right)+\underline{q}(\Pi \xi)=\underline{q}\left(\Pi^{\prime} \xi\right)+\frac{|\langle\underline{A} \xi, v\rangle|^{2}}{\underline{q}(v)}=\underline{q}\left(\Pi^{\prime} \xi\right)+|\langle\xi, \eta\rangle|^{2} \underline{q}(v) .
$$

LEMMA 5.4 Let us choose $\eta:=\underline{A} v$ as in (5.6) and $\boldsymbol{u} \in \mathcal{C}\left(Q^{\infty} ; \mathbb{R}^{2}\right)$ with $z:=Z(u)$; then

$$
\begin{aligned}
\underline{\phi}(v) & \leqslant \int_{Q^{\infty}}\|\Pi(\nabla z)\|_{\underline{q}} \mathrm{~d} x \leqslant\left(\int_{Q^{\infty}}\|\Pi(\nabla u)\|_{\underline{q}}^{2}+W(u)\right) \mathrm{d} x \\
& \leqslant \int_{Q^{\infty}} \boldsymbol{q}(\nabla \boldsymbol{u}(x))+W(u(x)) \mathrm{d} x ;
\end{aligned}
$$

in particular, $\underline{\phi}(v) \leqslant I(v)$.

Proof. We easily have

$$
\begin{aligned}
\int_{Q^{\infty}}(\underline{q}(\Pi(\nabla u))+W(u)) \mathrm{d} x & \geqslant \int_{Q^{\infty}} 2 \sqrt{\underline{q}(\Pi(\nabla u)) W(u)} \mathrm{d} x \\
& =\int_{Q^{\infty}}\|\Pi(\nabla u)\|_{\underline{q}}\left|Z^{\prime}(u)\right| \mathrm{d} x \\
& =\int_{Q^{\infty}}\|\Pi(\nabla z)\|_{\underline{q}} \mathrm{~d} x .
\end{aligned}
$$

Now we observe that (5.7) yields

$$
\|\Pi(\nabla z)\|_{\underline{q}}=|\langle\nabla z, \eta\rangle|\|v\|_{\underline{q}}=\left|\partial_{\eta} z\right|\|v\|_{\underline{q}}
$$

so that by (2.16) and the definition of $\phi$ in (1.33)

$$
\begin{aligned}
\int_{Q^{\infty}}\|\Pi(\nabla z)\|_{\underline{q}} \mathrm{~d} x & =\|v\|_{\underline{q}} \int_{Q^{\infty}}\left|\partial_{\eta} z\right| \mathrm{d} x \\
& \geqslant\|v\|_{\underline{q}} \int_{\Sigma}\left(Z\left(s_{+}\right)-Z\left(s_{-}\right)\right) \mathrm{d} H^{n-1}(x) \\
& =2 \sqrt{\underline{q}(v)} \int_{s_{-}}^{s_{+}} \sqrt{W(s)} \mathrm{d} s=\underline{\phi}(v)
\end{aligned}
$$


COROLlaRY 5.5 Let $\boldsymbol{u}^{k} \in \mathcal{C}\left(Q^{\infty} ; \mathbb{R}^{2}\right)$, with $z^{k}:=Z\left(u^{k}\right)$, be a family satisfying (5.4) and (5.5) as in Lemma 5.2; if $\phi(v)=\phi(v)$, then we have

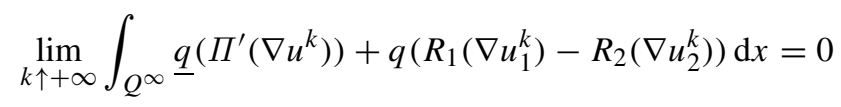

and

$$
\begin{aligned}
\lim _{k \uparrow+\infty} \int_{Q_{-}^{\infty}}\left\|\nabla z^{k}\right\|_{\underline{q}} \mathrm{~d} x & =\lim _{k \uparrow+\infty} \int_{Q_{+}^{\infty}}\left\|\nabla z^{k}\right\|_{\underline{q}} \mathrm{~d} x \\
& =\frac{1}{2} \lim _{k \uparrow+\infty} \int_{Q^{\infty}}\left\|\nabla z^{k}\right\|_{\underline{q}} \mathrm{~d} x=\frac{1}{2} \underline{\phi}(v)=\frac{1}{2} I(v) .
\end{aligned}
$$

Proposition 5.6 Let us assume that $\underline{\phi}(v)=\phi(v)$; then $v$ is a common eigenvector of $A_{1}$ and $A_{2}$.

Proof. Let us denote by $\Sigma_{t}$ the $t$-section of $Q^{\infty}$ :

$$
\Sigma_{t}:=\Sigma+t \eta
$$

Let us observe that (5.5), (2.16), and the definition of $\mathcal{C}\left(Q^{\infty} ; \mathbb{R}^{2}\right)$ entail for every $t>0$

$$
\begin{aligned}
\|v\|_{\underline{q}} \int_{\Sigma_{t}}\left|z^{k}(x)-Z\left(s_{+}\right)\right| \mathrm{d} H^{n-1}(x) & \leqslant\|v\|_{\underline{q}} \int_{Q_{+}^{\infty}}\left|\partial_{\eta} z^{k}\right| \mathrm{d} x \\
& \leqslant \int_{Q_{+}^{\infty}}\left\|\nabla z^{k}\right\|_{\underline{q}} \mathrm{~d} x \\
& \leqslant \frac{1}{2} \int_{Q^{\infty}}\left(\boldsymbol{q}\left(\nabla \boldsymbol{u}^{k}\right)+W\left(u^{k}\right)\right) \mathrm{d} x ;
\end{aligned}
$$

analogously, for $t<0$

$$
\|v\|_{\underline{q}} \int_{\Sigma_{t}}\left|z^{k}(x)-Z\left(s_{-}\right)\right| \mathrm{d} H^{n-1}(x) \leqslant \frac{1}{2} \int_{Q^{\infty}}\left(\boldsymbol{q}\left(\nabla \boldsymbol{u}^{k}\right)+W\left(u^{k}\right)\right) \mathrm{d} x .
$$

By standard compactness results, we can extract a suitable subsequence (still denoted by $\boldsymbol{u}^{k}$ ) such that

$$
\boldsymbol{u}^{k} \rightarrow \boldsymbol{u}, \quad z^{k} \rightarrow z=Z(u) \quad \text { in } L_{l o c}^{1}\left(Q^{\infty}\right) \text { and a.e., with } \boldsymbol{u}, z \in W_{l o c}^{1,1}\left(Q^{\infty}\right) .
$$

By lower semicontinuity, recalling $R_{i}=A^{-1} A_{i}$, we get

$$
\Pi^{\prime}(\nabla v)=0, \quad A_{1} \nabla v_{1}=A_{2} \nabla v_{2} .
$$

By the first relation of (5.10) we deduce the existence of a function $f \in W_{l o c}^{1,1}(\mathbb{R})$ such that

$$
u(x)=f(\langle x, v\rangle), \quad z=Z(f(\langle x, v\rangle)) \quad \text { a.e. in } Q^{\infty} .
$$


On $\Sigma_{t}$ we have

$$
z(y)=z(x+t \eta)=Z(f(t\langle\eta, v\rangle))=Z(f(t))
$$

so that

$$
\|v\|_{\underline{q}}\left|Z(f(t))-Z\left(s_{+}\right)\right| \leqslant \frac{1}{2} \underline{\phi}(v)=\frac{1}{2}\|v\|_{\underline{q}}\left(Z\left(s_{+}\right)-Z\left(s_{-}\right)\right)
$$

and in particular

$$
\left|Z(f(t))-Z\left(s_{+}\right)\right| \leqslant \frac{1}{2}\left(Z\left(s_{+}\right)-Z\left(s_{-}\right)\right) \quad \forall t>0 .
$$

Analogously,

$$
\left|Z(f(t))-Z\left(s_{-}\right)\right| \leqslant \frac{1}{2}\left(Z\left(s_{+}\right)-Z\left(s_{-}\right)\right) \quad \forall t<0 .
$$

If $f(t) \equiv f_{0}$ would be a constant function, then

$$
Z\left(f_{0}\right)=\frac{1}{2}\left(Z\left(s_{+}\right)+Z\left(s_{-}\right)\right), \quad \text { and therefore } s_{-}<f_{0}<s_{+}
$$

but this is not possible, since

$$
\int_{Q^{\infty}} W(u(x)) \mathrm{d} x=\int_{-\infty}^{+\infty} W(f(t)) \mathrm{d} t<+\infty .
$$

Thus $f$ is not constant. From the second relation of (5.10) we get

$$
\nabla v_{1}=R_{2} \nabla v=f^{\prime}(\langle x, v\rangle) R_{2} v, \quad \nabla v_{2}=R_{1} \nabla v=f^{\prime}(\langle x, v\rangle) R_{1} v
$$

Since $f$ is not constant, we deduce that $R_{2} v$ and $R_{1} v$ are parallel to $v$, i.e.

$$
A_{1} v, A_{2} v \text { are parallel to } A^{-1} v \text {. }
$$

\section{Acknowledgements}

This work was partially supported by grants of MURST (cofin9701226040, cofin9801229483), of CNR (contract nos 96.03847.PS01, 97.04704.PS01), and of Istituto di Analisi Numerica del CNR, Pavia, Italy.

\section{REFERENCES}

1. Ambrosio, L. Metric space valued functions of bounded variation. Ann. Sc. Norm. Sup. Pisa 17, (1990) 439-478.

2. Ambrosio, L. \& Braides, A. Functionals defined on partitions in sets of finite perimeter I: integral representation and $\Gamma$-convergence. J. Math. Pures Appl. 69, (1990) 285-305.

3. BALDO, S. Minimal interface criterion for phase transitions in mixtures of Cahn-Hilliard fluids. Ann. Inst. Henri Poincare, Anal. non Lineaire 7, (1990) 67-90. 
4. Barroso, A. C. \& Fonseca, I. Anisotropic singular perturbations-the vectorial case. Proc. R. Soc. Edinburgh 124A, (1994) 527-571.

5. Bellettini, G., Colli Franzone, P., \& Paolini, M. Convergence of front propagation for anisotropic bistable reaction-diffusion equations. Asymptotic Anal. 15, (1997) 325-358.

6. Bellettini, G. \& Paolini, M. Anisotropic motion by mean curvature in the context of Finsler geometry. Hokkaido Math. J. 25, (1996) 537-566.

7. Bellettini, G., Paolini, M., \& Venturini, S. Some results on surface measures in calculus of variations. Ann. Mat. Pura Appl., IV. Ser. (1996) 329-357.

8. Bouchitté, G. Singular perturbations of variational problems arising from a two-phase transition model. Appl. Math. Optim. 21, (1990) 289-314.

9. BRAides, A. \& Chiadó PiAT, V. Integral representation results for functionals defined on $S B V\left(\Omega ; \mathbb{R}^{m}\right)$. J. Math. Pures Appl. 75, (1996) 595-626.

10. Evans, L. C. \& GARIEPY, R. F. Measure Theory and Fine Properties of Functions, Studies in Advanced Mathematics. CRC Press, Boca Raton, Florida (1992).

11. Colli Franzone, P. \& Guerri, L. Spreading of excitation in 3-d models of the anisotropic cardiac tissue. Math. Biosci. 113, (1993) 145-209.

12. Colli Franzone, P., Guerri, L., \& Rovida, S. Wavefront propagation in an activation model of the anisotropic cardiac tissue: asymptotic analysis and numerical simulations. J. Math. Biol. 28, (1990) 121-176.

13. Colli Franzone, P., Guerri, L., \& Tentoni, S. Mathematical modeling of the excitation process in myocardial tissue: influence of fibre rotation on wavefront propagation and potential field. Math. Biosci. 101, (1990) 155-235.

14. Colli Franzone, P. \& Savaré, G. Degenerate evolution systems modeling cardiac electric field at micro and macroscopic level. IAN-CNR Pavia (1996) 1007.

15. Dal Maso, G. An Introduction to $\Gamma$-Convergence, Progress in Nonlinear Differential Equations and Their Applications, vol. 8. Birkhäuser, Boston (1993).

16. DAL MASo, G. \& ModicA, L. A general thory of variational functionals, in topics in functional analysis 1980-81. Quaderni Scuola Norm. Sup. Pisa (1981) 149-221.

17. De Giorgi, E. \& LetTA, G. Une notion générale de convergence faible pour des fonctions croissantes d'ensemble. Ann. Scuola Norm. Sup. Pisa 4, (1977) 61-99.

18. FitzHugh, R. Mathematical Models of excitation and propagation in nerve. In: Schwan, H. P. (ed), Biological Engineering. MacGraw-Hill, New York (1969).

19. Giaquinta, M. \& Hildebrandt, S. Calculus of Variations I, Grundlehren der mathematischen Wissenschaften, vol. 310. Springer, Berlin (1996).

20. Grisvard, P. Elliptic Problems in Nonsmooth Domains. Pitman, London (1985)

21. Henriquez, C. S. Simulating the electrical behaviour of cardiac tissue using the bidomain model. Crit. Rev. Biomed. Engr. 21, (1993) 1-77.

22. ,JACK, J. J. B., NOBLE, D., \& TSIEN, R. W. Electric Current Flow in Excitable Cells. Oxford University Press, Oxford (1975)

23. Keener, J. P. \& PAnfilov, A. V. Three-dimensional propagation in the heart: the effects of geometry and fibre orientation on propagation in myocardium. In: ZIPES, D. P. \& JALIFE, J. (eds), Cardiac Electrophysiology: From Cell to Bedside. pp. 335-347. WB. Sounders Co, Philadelphia (1995).

24. KeEner, J. P. \& PANfilov, A. V. The effects of geometry and fibre orientation on propagation and extracellular potentials in myocardium. In: PANFILOV, A. V. \& HoldEN, A. V. (eds), Computational Biology of the Heart, Chapter 8. pp. 235-258. John Wiley \& Sons, New York (1997).

25. Keener, J. P. \& Sneyd, J. A Mathematical Textbook of Physiology. Springer, New York (1998).

26. ModicA, L. Gradient thory of phase transitions and minimal interface criterion. Arch. Rational Mech. Anal. 98, (1986) 123-142.

27. Modica, L. \& Mortola, S. Un esempio di $\Gamma$-convergenza. Boll. Un. Mat. Ital. B 14, (1977) 285-299. 
28. OWEn, N. \& Sternberg, P. Nonconvex variational problems with anisotropic perturbations. Nonlinear Anal. TMA 78, (1991) 705-719.

29. Rотн, B. J. How the anisotropy of the intracellular and extracellular conductivities influence stimulation of cardiac muscle. J. Math. Biol. 30, (1992) 633-646.

30. Soravia, J. P. \& Souganidis, P. E. Phase-field theory for Fitzhugh-Nagumo type systems. SIAM J. Math. Anal. 27 (5), (1996) 1341-1359. 